\title{
Posteriori Reconstruction and Denoising for Path Tracing
}

by

Ping Liu

\author{
A thesis \\ submitted to the Victoria University of Wellington \\ in fulfilment of the \\ requirements for the degree of \\ Doctor of Philosophy \\ in Computer Science.
}

Victoria University of Wellington 2019 



\begin{abstract}
Path tracing is a well-established technique for photo-realistic rendering to simulate light path transport. This method has been widely adopted in visual effects industries to generate high quality synthetic images requiring a large number of samples and a long computation time. Due to the high cost to produce the final output, intermediate previsualization of path tracing is in high demand from production artists to detect errors in the early stage of rendering. But visualizing intermediate results of path tracing is also challenging since the synthesized image with limited samples or improper sampling usually suffers from distracting noise. The ideal solution would be to provide a highly plausible intermediate result in the early stages of rendering, using a small fraction of samples, and apply a posteriori manner to approximate the ground truth.

In this thesis, this issue is addressed by providing several efficient posteriori reconstructions and denoising technique for previsualization of path tracing. Firstly, we address the problem for the recovery of the missing values to construct low rank matrices for incomplete images including missing pixel, missing sub-pixel, and multi-frame scenarios. A novel approach utilizing a convolutional neural network which provides fast precompletion for initializing missing values, and subsequent weighted nuclear norm minimization with a parameter adjustment strategy efficiently recovers missing values even in high frequency details. The result shows better visual quality compared to the recent methods including compressed sensing based reconstruction.

Furthermore, to mitigate the computation budgets of our new approac$\mathrm{h}$, we extend our method by applying a block Toeplitz structure forming
\end{abstract}


a low-rank matrix for pixel recovery, and tensor structure for multi-frame recovery. In this manner, the reconstruction time can be significantly reduced. Besides that, by exploiting temporal coherence of multi-frame with a tensor structure, we demonstrate an improvement in the overall recovery quality compared to our previous approach.

Our recovery methods provide satisfying solution but still require plenty of rendering time at prior stage compared with denoising solutions. Finally, we introduce a novel filter for denoising based on convolutional neural network, to address the problem as conventional denoising approach for rendered images. Unlike a plain CNN that applies fixed kernel size in each layer, we propose a multi-scale residual network with various auxiliary scene features to leverage a new efficient denoising filter for path tracing. Our experimental results show on par or better denoising quality compare to state-of-the-art path tracing denoiser. 


\section{Acknowledgments}

I would like to express my most sincere thanks to all those who supported and helped me during my $\mathrm{PhD}$ study.

I am extremely thankful to my honorable supervisors, Assoc.Prof. Taeyhun Rhee, Assoc.Prof. John Lewis and Dr. Alexander Doronin, for providing me greatly helpful guidances to my research, academic writing sills, presentation skills and my personal life as well. Under their supervision, I was inspired to bring up new ideas and develop critical thinking. In addition, I also appreciated that they spent a lot of energy and time on revising my paper and thesis, and their valuable feedbacks have a huge influence on my PhD work. Without their guidance and support, my research tasks cannot be completed smoothly. I could not imagine having better supervisors for my research and life.

Additionally, I also like to thank all the wonderful people at the VUW computer graphics lab, who accompanied and collaborated with me during the past three years.

Lastly, I would highly appreciate the support of my parents, my brother and friends, I could not have done this without the encouragement from them. I also wish to thank the Victoria University of Wellington scholarship, which gave me financial support during my study. 



\section{List of Figures}

2.1 The rendering equation describes the total amount of radiance from a surface point $x$ along a viewing direction and can be computed by adding the emission of and the integration of the incoming radiance over the hemisphere of the point, weighted by the BRDF term. . . . . . . . . . . . . 11

3.1 Low-rank matrix construction for pixel patch: from the left, the input image (20\% pixel samples), pre-completion and grouping, and low-rank matrix $M_{p}$. From the initialized image (middle), similar pixel patches (red squares) for a current pixel patch (green square) are selected, and stored into columns of the matrix $M_{p} \ldots \ldots \ldots \ldots \ldots$

3.2 Three layered CNN architecture for pre-completion: the red square is a convolutional filter in layer $1\left(W_{1}\right)$, the green square is a convolutional filter in layer $2\left(W_{2}\right)$, and the blue square is a convolutional filter in layer $3\left(W_{3}\right) \ldots \ldots \ldots$

3.3 A visual quality comparison of initialisation between Total Variation and our $\mathrm{CNN}$ for a toasters scene, and the corresponding recoveries. Our recovery also achieve better recovery in terms of SSIM (the structural similarity) and PSNR. 34

3.4 An example of multi-frame patches $F_{k}$ and the constructed low-rank matrix $M_{m}$ : similar patches across multiple frames (squares) are stored in columns of a low-rank matrix. . . . . 36 
3.5 A sub-pixel grid $G_{s}$ (green) and $3 \times 3$ sub-pixel patch $P_{s}$ (red) fed into a low-rank matrix $M_{s}$ : black dots are rendered samples. . . . . . . . . . . . . . . . . 38

3.6 In a sub-pixel area, an edge may be difficult to localize correctly (left); the orange dots and the blue square(s) are rendered samples around an edge. But the orientation of the edge (purple line) becomes more clear when a sub-pixel patch is formed (right), even when the ratio of known-tounknown samples remain constant. . . . . . . . . . . . 39

3.7 The corresponding PSNR of three test scenes with different patch size choices. . . . . . . . . . . . . . . . . . 42

3.8 Examples of missing pixel recovery $(10 \%, 20 \%, 40 \%$ pixel samples). ...................... 43

3.9 A visual quality comparison of CR (Compressive Rendering), CIR (Compressive Image Reconstruction), NNM (nuclear norm minimization) and our PAWNNM. Input: $40 \%$ pixel samples. . . . . . . . . . . . . . . . . 44

3.10 A visual quality comparison of NLI, GSR, NNM and our proposed PAWNNM. Input: 20\% pixel samples. . . . . . . . 47

3.11 Example of multi-frame recovery (with PAWNNM) under different sampling rate. . . . . . . . . . . . . . 48

3.12 Examples of sub-pixel recovery under different sampling rates $(10 \%$ and $25 \%)$, From the top, input image rendered by 25 sub-pixels per pixel (spp) and 64 spp, our PAWNNM reconstructions, and the reference image rendered by 256 spp. The unit of rendering time (top and bottom), and reconstruction time is seconds. . . . . . . . . . . . . . 51

3.13 Comparison with filtering methods (SURE [1], RHF [2] , LBF [3]), and our reconstruction schemes with PAWNNM under the same input samples (sub-pixels per pixel (spp)). . 53 
3.14 Equal time comparison with filtering methods (RHF, LBF), and our reconstruction schemes (PAWNNM). . . . . . . . 54

3.15 A comparison between our pixel recovery and sub-pixel recovery under a same sampling rate $(20 \%) \ldots \ldots$. . . . . . . 56

3.16 Under a fixed time budget (1.8 hours), our pixel recovery (in the middle) can generate better visual quality results than filter based method, RHF (on the right). . . . . . . . . . . 58

4.1 Given a 5 by 5 matrix, a corresponding BTM can be constructed from it (see Equation (4.4) and (4.5)). . . . . . . . . . 63

4.2 Given a selected pixel patch $P_{i}$ from an incomplete path tracing image, a corresponding BTM can be constructed (see Equation (4.4) and (4.5)) . . . . . . . . . . . . . 64

4.3 A tensor structure consists of a number of adjacent animation frames: $\left\{F_{1}, F_{2}\right.$, ,$\left.F_{i}\right\}$.

4.4 Given a selected patch, the block Toeplitz matrix is able to obtain a low rank property, similar to the patch grouping approaches; tested using a different percentage of randomly distributed input pixels $(20 \%, 40 \%$, and $100 \%$ as a reference). 73

4.5 Recovery quality and time of an incomplete path tracing image (San Miguel scene with $1024 \times 1024$ resolution with $40 \%$ pixels) under different patch sizes using a BTM; we select $25 \times 25$ to balance quality and recovery time. . . . . . . . 73

4.6 The PSNR of progressive recovery using iterative WNNM, going up to 100 iterations. This uses the Sanmiguel scene with $40 \%$ rendered pixels. . . . . . . . . . . . . 74

4.7 Examples of missing pixel recovery for sparsely rendered images with different sampling rates $(10 \%, 20 \%, 40 \%)$. . . . 74

4.8 The recovery comparison with CR (Compressive Rendering), CIR (Compressive Image Reconstruction), PAWNNM, and our method. . . . . . . . . . . . . . . . . . 75 
4.9 The recovery comparison of CR (Compressive Rendering), CIR (Compressive Image Reconstruction), PAWNNM, and our method. . . . . . . . . . . . . . . . . . 75

4.10 Examples of repairing damaged photographs. Compared with the previous method [4], our methods takes less time to recover the damaged pixels with slightly better quality. . 76

4.11 Example of our proposed TCR and PAWNNM under different sampling rate. . . . . . . . . . . . . . . . . 79

5.1 In a noisy path tracing (the top), the noise level is various in different image regions; the noise level in the region of blue window is higher than the region of red window. In contrast, the area in the green window contains less noise. The bottom figure is the histogram distributions of the residual from the three different patches, showing near Gaussian distributions. . . . . . . . . . . . . . . 86

5.2 An example of noise artifacts in image rendered with only 8 samples per pixels (left image). The noise artifacts are caused by either extreme larger (green square) or smaller (yellow square) pixel value compared to the corresponding pixel in the reference rendering. . . . . . . . . . . 87

5.3 Overview of the proposed denoising pipeline. The input of the network consists of a noise rendering, normal vector, depth information and virtual flash image buffer. The input is fed to two residual networks for prediction of the bright and dark channel residual separately. The denoised image is achieved by combining the residual images with the noise rendering. . . . . . . . . . . . . . . . 89 
5.4 The multi-scale structure in the convolutional layer of our network. Outputs from the previous layer are convolved with different size filters to generate new features maps. Then, group the new feature maps using Maxout activation to generate the outputs feature maps. . . . . . . . . . . . . 90

5.5 Evaluation of the learning performance with different loss functions. The network is trained with $L_{1}$ loss and $L_{2}$ loss separately to compute the average PSNR (peak signal-tonoise ratio) on 7 tested images. With a comparison, $L_{1}$ loss is more efficient than $L_{2}$ loss. . . . . . . . . . . . . . . 91

5.6 Top row (from the left): a noisy rendering produced with limited samples, and the corresponding virtual flash rendering. Bottom row: histograms of the reference, noise, and virtual flash image. . . . . . . . . . . . . .

5.7 Training error of the proposed bright and dark channel network trained on $L_{1}$ loss function. . . . . . . . . . . . . 95

5.8 We introduce dual channel residual learning with multiscale convolutional neural networks for denoising path tracing renderings. We trained networks for single channel residual learning (SRS) and dual channel residual learning (DRS) using a single fixed scale $\mathrm{CNN}$, a dual channel residual using a multi-scale CNN with (DRMF) and without the virtual flash image (DRM). The noisy input is rendered with $32 \mathrm{spp}$ (samples per pixel), while the reference image is rendered with $64 \mathrm{k} \mathrm{spp}$. Our proposed DRMF closely resembles the reference. . . . . . . . . . . . . . 96

5.9 Comparison of LBF, KPCN and our proposed denoising network $(\mathrm{DRMF}) \ldots \ldots \ldots \ldots$. . . . . . . . . . . 98

5.10 A visual quality comparison of RHF, NFOR and our proposed denoising network $(\mathrm{DRMF}) . \ldots$. . . . . . . . . . 99 
5.11 Under a fixed time budget, pixel recovery (in the middle) can generate better visual quality results than the proposed denoising network(on the right). . . . . . . . . . . . 102 


\section{List of Tables}

3.1 PSNR compared with CNN and TV initialization under $10 \%$ input pixels. The recovery time are measured in seconds. . . 33

3.2 PSNR comparison for NNM, WNNM and PAWNNM under $20 \%$ input pixels. . . . . . . . . . . . . . . . . . 40

3.3 PSNR/SSIM results of three test scenes under different sampling rates. . . . . . . . . . . . . . . . . . 4 45

3.4 Relative reconstruction cost comparison under different sampling rates. . . . . . . . . . . . . . . . 45

3.5 Given a fixed time budget (17 minutes), the PSNR of the recovery under CR, CIR and PAWNNM. . . . . . . . . . . 46

3.6 PSNR/SSIM results of inpainting applications. . . . . . . . . 49

3.7 Result of multi-frame recovery (with PAWNNM): Incomplete video frames sampled at $10 \%, 20 \%$ and $40 \%$ are reconstructed independently (single-frame) and 7-frame concurrently (multi-frames). Time is measured in minutes. . . . 50

3.8 The time cost in equal time comparison test: RHF, LBF (rendering time/ filtering time) and ours PAWNNM (rendering time/ recovery time) . . . . . . . . . . . . . . . 52

4.1 PSNR/SSIM results of three test scenes under different input pixel sampling rates. . . . . . . . . . . . . . . 77 
4.2 Recovery performance measured by relative recovery cost with respect to the total rendering time. The reference image is rendered in 32 hours. . . . . . . . . . . . 78

4.3 Result of multi-frame recovery (with TCR): Incomplete video frames sampled at $10 \%, 20 \%$ and $40 \%$ are reconstructed independently (single-frame) and 10-frame concurrently (multiframes). Time is measured in minutes. . . . . . . . . . . . 80

5.1 Denoising results of multi-scale CNN in different kernel size. 94

5.2 PSNR/SSIM results of our methods (SRS, DRS, DRM, and DRMF), LBF and KPCN. . . . . . . . . . . . . . . . 100

5.3 PSNR/SSIM results of our methods (SRS, DRS, DRM, and DRMF), RHF and NFOR. . . . . . . . . . . . . . 100 


\section{Contents}

1 Introduction 1

1.1 Motivations and Problem Statement . . . . . . . . . . . . 1

1.2 Thesis Contributions . . . . . . . . . . . . . . . . . 4

1.3 Thesis Outline . . . . . . . . . . . . . . . 6

2 Background and Related work 9

2.1 Path Tracing . . . . . . . . . . . . . . . . 9

2.2 Related Work . . . . . . . . . . . . . . . . . . 13

2.2.1 Reconstruction Techniques for Path Tracing . . . . . . 13

2.2.2 Denoising Techniques for Path Tracing . . . . . . . 19

3 Matrix Completion for Path Tracing 25

3.1 Overview . . . . . . . . . . . . . . . . . . . 28

3.2 Low-Rank Matrix Construction . . . . . . . . . . . . . 30

3.2.1 Pixel Patch . . . . . . . . . . . . . 31

3.2.2 Multi-frame Patch . . . . . . . . . . . 35

3.2.3 Sub-pixel Patch . . . . . . . . . . . . . . 35

3.3 Low-rank Matrix Recovery . . . . . . . . . . . . . . . 37

3.4 Results . . . . . . . . . . . . . . . . . . . 40

3.4.1 Experimental Setup . . . . . . . . . . . . . 40

3.4 .2 Pixel Recovery . . . . . . . . . . . . . . . . . . . . . . . . . . . . .

3.4.3 Multi-frame Recovery . . . . . . . . . . . . . . . . 49

3.4.4 Sub-pixel Recovery . . . . . . . . . . . . . . 50 
3.5 Summary and Discussion . . . . . . . . . . . . 52

4 Enhanced Completion for Path Tracing 59

4.1 Overview ....................... 61

4.1 .1 Basic Notation . . . . . . . . . . . . . . . 61

4.1.2 Enhanced Matrix Completion . . . . . . . . . . . 61

4.2 Low Rank Matrix from an Image . . . . . . . . . . . . . 62

4.2.1 Block Toeplitz Matrix (BTM) . . . . . . . . . . . 62

4.2.2 Create BTM from an Image . . . . . . . . . . . . . . 64

4.3 Tensor Completion . . . . . . . . . . . . . . . . 65

4.3.1 Multi-frame Tensor . . . . . . . . . . . . . 66

4.4 Enhanced Matrix and Tensor Recovery . . . . . . . . . . . . . 67

4.4.1 Pixel Recovery via Enhanced Matrix Completion . . 67

4.4.2 Multi-frame Recovery via Tensor Completion . . . . 69

4.5 Results . . . . . . . . . . . . . . . . . . . 72

4.5 .1 Pixel Recovery . . . . . . . . . . . . . 73

4.5.2 Multi-frame Recovery . . . . . . . . . . . . . 78

4.6 Summary and Discussion . . . . . . . . . . . . 80

5 Denoising Network for Path Tracing 83

5.1 Residual Learning for Denoising Path Tracing . . . . . . . 85

5.2 Dual Channel Residual Learning . . . . . . . . . . . . . 85

5.3 Multi-scale CNN . . . . . . . . . . . . . . . 88

5.3.1 Network Architecture . . . . . . . . . . . . . . 89

5.3 .2 Loss Function . . . . . . . . . . . . . . . . 91

5.3 .3 Training Data . . . . . . . . . . . . . . . 92

5.3 .4 Virtual Flash Image . . . . . . . . . . . . . . . . . . . . . . . . . . . .

5.3 .5 Training Details . . . . . . . . . . . . . . . . . 94

5.4 Results . . . . . . . . . . . . . . . . . 95

5.4.1 Denoising Performance ............ . 95

5.4.2 Performance Comparison . . . . . . . . . . . 97

5.4 .3 Efficiency comparison ............. 100 
5.5 Summary and Discussion . . . . . . . . . . . . . 101

6 Conclusions and Future Work 103

6.1 Summary of Contributions . . . . . . . . . . . . . 105

6.2 Limitations . . . . . . . . . . . . . . . . . 106

6.3 Future Work . . . . . . . . . . . . . . . . 107

$\begin{array}{lll}\text { Appendix A Pre-completion with TV and CNN } & 109\end{array}$

Appendix B PAWNNM for Inpainting 115 


\section{Chapter 1}

\section{Introduction}

\subsection{Motivations and Problem Statement}

In the visual effects industries, rendering is one of the most crucial and time-consuming tasks. As a general estimate, rendering one frame in production quality could require hours or days in today's computer animated movies, and the time of rendering in production keeps increasing for realism and fidelity.

The core process of rendering is to simulate all possible light paths in a virtual scene, which has been well addressed by path tracing in computer graphics. Path tracing is usually evaluated by Monte Carlo integration technique, which is a general approach for computing the integral over all light paths. Monte Carlo integration technique can robustly estimate the high-dimensional integrals in the rendering equation [5], making it a powerful tool for computing the light transport in complex scenes. However, Monte Carlo integration is also known to be inefficient due to its relatively slow converging rate of $\frac{1}{\sqrt{N}}$, here $N$ is the number of samples. Using insufficient samples causes undesirable artifacts in the final image (e.g., noise, blur, and banding). A naive path tracing algorithm often requires a large number of samples to be drawn and evaluated in order to reconstruct a high quality image, but a long rendering time is required accordingly. Due 
to the high cost to produce the final output, intermediate previsualization of path tracing is in high demand from production artists to detect errors in the early stage of rendering.

Addressing this problem is challenging, various approaches have been presented to address the noise or variance reduction issue. Such as efficient sampling patterns (importance sampling, Metropolis sampling), statistical analysis models (density estimation, variance control), and image processing techniques (frequency domain analysis, non-linear filtering).

In this thesis, rather than pursuing analysis of light transport equation and sampling pattern, we explore posteriori approaches that provide a highly plausible intermediate result in the early stages of rendering, using a small fraction of samples, and apply a post process on the rendered data to efficiently approximate the ground truth rendering. We address the problem as reconstruction and denoising.

Reconstruction: It exploits the coherence of the visual signal to reconstruct missing samples based on partially and randomly rendered ones. Several previous techniques have been proposed.

Recent inpainting methods in image processing show promising results to recover missing pixels from limited known pixel samples [6, 7]. However, these methods have limitations when used for previsualization of path tracing. Due to the random nature of rendered pixels, the methods cannot provide the localized scene structure required to prevent boundary edge blurring. Recent example based inpainting methods address the issue more efficiently, but they often suffer from long recovery time $[6,4,8]$; one of the important requirements for previsualization is to show believable intermediate results within an acceptable time.

Recent compressive rendering (CR) has been proposed as a previsualization solution by Sen et al. [9]. CR is based on compressed sensing (CS), which can reconstruct an unknown signal from a few samples provided the signal has a sparse representation in some basis. CR shows believable reconstruction of missing pixels in an incompletely rendered image within 
an acceptable time. However, it has limitations, when the sampling rate is low (like 10\%), and the final output quality relies on the choice of basis in the transform domain.

Denoising: A large amount of works have been proposed to render a noisy result in a quick manner with low sample budget and then apply a denoising filter to output the noise-free result $[10,11,12,13,14,15]$. These methods are scene feature based filters, like the non-local mean filter and bilateral filter, which can utilize additional scene feature buffers (e.g., world position, depth information) to guide the denoising process. Since the additional scene information is closely related to scene details, these features based denoising filters could achieve a good quality result. However, the primary challenge is how to set the filter weights for different features properly. Many approaches use different error estimation metrics to generate the optimum filter parameters. Since the error metrics are noisy under a low sample budget, the accuracy of the selected parameters are not the optimum. Therefore, the denoised results from these methods may suffer from the over-blurred area, especially in regions with high-frequency details.

Based on the analyses of this section, the issues/questions that deserve investigation are described as follows.

- Reconstruction based methods have demonstrated the ability to augment various rendering algorithms and scenes as a postprocessing step. The remaining technical issue is how to generate visually believable result under the consideration of both low sampling rate and short recovery time.

- As denoising based filters can utilize additional scene features, the main issue is how to provide suitable filter parameters with regard to various scene features. More importantly, a denoising filter work robustly and efficiently even with low sampling budget.

Applying these challenges to the path tracing previsualization prob- 
lem, we have the following objectives to investigate:

1. Develop reconstruction based method for previsualization of path tracing in feature film production. Existing reconstruction based methods fall into two categories, inpainting and compressed sensing based methods. Specifically, inpainting methods often suffer from long recovery time that fails to show believable intermediate results within an acceptable time. In contrast, compressed sensing based methods mainly work well under a high sampling rate. Therefore, the new reconstruction based framework will aim for both efficiency and robustness.

2. Design and develop new denoising based previsualization method, which can robustly handle suitable parameters for multiple scene features. Although a wide range of denoising techniques of path tracing have been proposed, how to perform consistently well and easy of adoption is still an issue worth exploiting.

\subsection{Thesis Contributions}

In this thesis, our goal is to provide efficient and optimal postprocessing methods for previsualization of path tracing. The problem is addressed in two manners: reconstruction and denoising. The main difference of the two categories is that reconstruction based methods restore the missing or unrendered samples based on rendered ones, while denoising based methods remove the noise through adjusting the rendered pixel values.

To develop reconstruction based methods, we exploit the coherence of the visual signal to reconstruct missing samples, using a low-rank matrix completion and tensor completion framework. Matrix completion and tensor completion have been recently introduced $[16,17,18]$ and successfully applied to many problems in image processing $[19,20,21]$, but have not been adopted in the computer graphics community. Applied to our problem, the basic strategy is to construct a matrix from a group of similar 
pixels or patches, and then use matrix completion to estimate the missing samples. Additionally, applying the tensor structure to represent multiframes and recover missing samples by tensor completion.

To explore more complex and general denoising based method, we utilize a deep learning based denoising filter that leverages convolutional neural network $(\mathrm{CNN})$ architecture. The $\mathrm{CNN}$ has proven the ability to learn complex non-linear models. Once the neural network is trained, it can be easily and efficiently applied without complex parameter selection. Such a framework can more efficiently handle the task of previsualization of path tracing in real feature film production.

The contributions of this thesis include new algorithms and new analysis, which can be summarized as follows:

- Chapter 3 formalizes the recovery of missing data in incompletely rendered images as a matrix completion problem. Specifically, we adopt the low-rank matrix completion scheme for previsualization of path tracing. Although matrix completion and separation have been used for many lights rendering [22] and inpainting [4] recently, they have not yet been applied to recover sub-pixel samples of rendering or pixel samples in multiple frames. To form a low-rank matrix, we show how to formulate pixel, sub-pixel, and multi-frame sample problems as matrix completion. Our method contributes to reducing spatial and temporal noise in reconstructed images.

Additionally, we have developed novel nuclear norm minimization methods using a multi-weighted approach (WNNM) with a progressive adjustment strategy (PAWNNM). Our method has been evaluated against state-of-the-art methods and shows better results regarding visual quality including CS based reconstruction. In particular, our method outperforms with limited samples (e.g., $\leq 40 \%$ ).

The work in this chapter was published in the following paper:

P. Liu, J. Lewis and T. Rhee, "Low-Rank Matrix Completion to Re- 
construct Incomplete Rendering Images," in IEEE Transactions on Visualization and Computer Graphics, vol. 24, no. 8, pp. 2353-2365, 1 Aug. 2018.

- Based on Chapter 3, we present novel methods in Chapter 4 to construct enhanced low-rank matrices and low-rank tensor for dealing with missing pixel and multi-frame scenarios respectively. Specifically, we adopted the latest enhanced matrix completion in pixel recovery and tensor completion for the multi-frame case. Then we apply weighted nuclear norm minimization with a parameter adjustment strategy, which can more efficiently recover missing values even in high-frequency details.

- In Chapter 5, we present a novel denoising approach using convolutional neural network. Motivated by recent deep learning-based works, we apply a denoising network based on multi-scale residual learning. Unlike the previous $\mathrm{CNN}$ architectures, which employ a single scale filter in a convolutional layer or perform multi-scale concept through down/up scaling the feature maps, we use different filters during the convolution step to preserve both long and short contextual information. Additionally, we develop a separated residual learning strategy to predict the noise. Also we generate a virtual flash image as one of the input scene feature buffers to enhance the denoising performance in high-frequency area. Our method shows on par or better denoising quality compare to the state-of-the-art methods.

\subsection{Thesis Outline}

The rest of this thesis is organized as follows: Chapter 2 presents the background information and related works. Chapter 3 to Chapter 5 present major contributions of this thesis aiming to achieve efficient previsualiza- 
tion for path tracing. Additionally, Chapter 6 presents conclusions and future directions.

- Chapter 2 provides a brief introduction of path tracing. Existing reconstruction based works and denoising based works are reviewed, which form the motivation of the work in this thesis.

- Chapter 3 introduces a low-rank matrix completion based reconstruction method for previsualization of path tracing. This method is described to handle sub-pixel, pixel and multi-frame recovery.

- Chapter 4 is built upon Chapter 3, which introduces enhanced matrix completion to reduce the computation time for pixel recovery in the previous chapter. In addition, a tensor completion based reconstruction method is proposed to achieve a more efficient and accurate recovery for the multi-frame case.

- Chapter 5 describes a new convolutional neural network based denoising filter. Specifically, we introduce a multi-scale filter based residual learning network for path tracing previsualization. This denoising method brings the power of neural work to remove noise from path tracing

- Chapter 6 provides major conclusions to the objectives achieved in this work along with limitations and several avenues for future research enabled by this thesis. 


\section{Chapter 2}

\section{Background and Related work}

In this chapter, we start with a review of the background of path tracing (Section 2.1). The work described in this thesis takes a two-pronged postprocess to accelerate previsualization of path tracing: (1) partially rendering a set of samples and recovering the missing ones based on the captured samples; (2) a denoising filter based on deep learning which filters the noise image rendered with a few samples per pixel. We review relevant works in two areas: reconstruction based techniques for recovering missing samples (Section 2.2), and denoising filter based techniques for removing the path tracing noise (Section 2.3). Given the topic of this thesis, the emphasis in this chapter will be previous works relating to reconstruction and denoising techniques.

\subsection{Path Tracing}

Rendering an image which simulates global illumination is a basic issue concerning the computation of pixel values. The pixel value refers to the radiance shooting from the visible surface point to the viewing camera. In addition, the radiance represents a function $L(x \rightarrow \omega)$ which satisfies the 
following rendering equation:

$$
L(x \rightarrow \omega)=L_{e}(x \rightarrow \omega)+\int_{\Omega} f_{r}\left(x, \omega^{\prime}, \omega\right) L\left(x \leftarrow \omega^{\prime}\right) \cos \left(n_{x}, \omega^{\prime}\right) d \sigma\left(\omega^{\prime}\right) .
$$

According the equation, the amount of energy $L(x \rightarrow \omega)$ reflected from the object surface $x$ to the direction $\omega$ that can be equivalent to the emitted radiance $L_{e}(x, \omega)$, with addition of the hemisphere integral on the received radiance $L(x \leftarrow \omega)$ that is weighted by the cosine of the angle between the normal vector $n_{x}$ and $\omega^{\prime}$, and the BRDF term $f_{r}$. Finally, it measures the integration of solid angle. Figure 2.1 illustrates the core concept of the rendering equation. Sometimes Equation (2.1) could also be called the solid angle formulation of the rendering equation and use of the equivalent surface-area formulation is better, wherein incoming radiance is parameterized at surfaces instead of the directions.

$$
L_{o}(x \rightarrow \omega)=L_{e}(x \rightarrow \omega)+\int_{M} f_{r}\left(x, \omega^{\prime}, \omega\right) L\left(x^{\prime} \rightarrow x\right) G\left(x \leftrightarrow x^{\prime}\right) d A\left(x^{\prime}\right) .
$$

Here, $x^{\prime}$ denotes the surface point, while $A\left(x^{\prime}\right)$ denotes the measure of the surface area. The geometry term $G\left(x \leftrightarrow x^{\prime}\right)$ is defined as follows:

$$
G\left(x \leftrightarrow x^{\prime}\right)=\frac{\cos \theta_{x} \cos \theta_{x^{\prime}}}{\left\|x-x^{\prime}\right\|} V\left(x, x^{\prime}\right) .
$$

where $V\left(x ; x^{\prime}\right)$ is visibility term, and it equals to 1 when two surface points $x$ and $x^{\prime}$ are visible between each other. Otherwise it will be 0 . According to the rendering equation, reasons for difficulty of the problem can be found. Firstly, it is global since the energy of the outgoing radiance from a surface point is decided by the incoming radiance from various surface points. Secondly, the integral is continuous and should be described into sum. In the end, the light may recursively arrive at the camera by different times of bounces.

We can also express the rendering equation (2.1) in a path integration form. This form can derive algorithms which can be hardly established from Equation (2.1). 


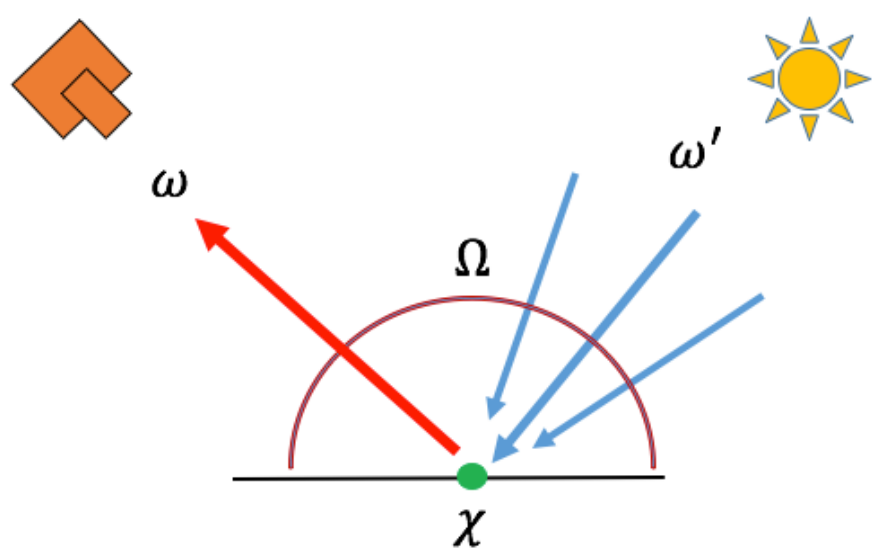

Figure 2.1: The rendering equation describes the total amount of radiance from a surface point $\mathrm{x}$ along a viewing direction and can be computed by adding the emission of and the integration of the incoming radiance over the hemisphere of the point, weighted by the BRDF term.

Through a given pixel $j$, the illumination $I_{j}$ is denoted by

$$
I_{j}=\int_{\Omega} f_{j}(\bar{x}) d_{\mu}(\bar{x})
$$

Where $x=x_{0} x_{1} \ldots x_{n}$ denotes a complete light path. For example, $x_{0}$ is the point from a light source and $x_{n}$ denotes the viewing camera. The measurement of surface area on each point $x_{i}$ is defined as $\mu(\bar{x})$ The contribution $f_{j}(\bar{x})$ can be generated from the appropriate light emission, geometry terms, BRDFs and sensor response:

$$
\begin{array}{r}
f_{j}(\bar{x})=E(x \rightarrow \omega) G\left(x_{0} \leftrightarrow x_{1}\right) f_{r}\left(x_{0} \rightarrow x_{1} \rightarrow x_{2}\right) G\left(x_{1} \leftrightarrow x_{2}\right) \ldots \\
\ldots f_{r}\left(x_{n-2} \rightarrow x_{n-1} \rightarrow x_{n}\right) G\left(x_{n-1} \leftrightarrow x_{n}\right)
\end{array}
$$

Theoretically, each surface from the scene is emissive and reflective, but this rarely happens. As for a more frequent scenario, there are distinct light sources and only the illumination of them is reflected by the scene. Based 
on this fact, global illumination is often divided into direct illumination $L_{d}$, emission $L_{e}$, and indirect illumination $L_{i}$ :

$$
L(x \rightarrow \omega)=L_{e}(x \rightarrow \omega)+L_{d}(x \rightarrow \omega)+L_{i}(x \rightarrow \omega),
$$

here, the direct illumination refers to the total radiance over the surface from $n$ light sources:

$$
L_{d}(x \rightarrow \omega)=\sum_{i=1}^{n} \int_{A_{i}} f_{r}\left(x, \omega^{\prime}, \omega\right) L(y \rightarrow x) G(x \leftrightarrow y) d A(y),
$$

where $A_{i}$ denotes the area involved by the $i$-th light source. In general, the direct illumination can be deemed as a much easier problem in comparison with the indirect illumination. This is correct in most cases when the set of emissive surfaces under the situation comprise a few numbers of area lights or point lights. But many small light sources, large area light and scenes with environment maps often appear. In many-light formulation, all these situations are represented by many point lights. Based on certain proper probability distribution $p(x)$, Monte Carlo integration can generally compute the integral $I$ concerning the function $f(x)$ towards the domain $D$ with $N$ random samples:

$$
I=\int_{D} f(x) d x \approx \frac{1}{N} \sum_{i=1}^{N} \frac{f\left(x_{i}\right)}{p\left(x_{i}\right)} .
$$

This is an unbiased method, since the expected value of the result equals to the computed integral. Ideally, a large number of samples could approximate the value of the integral.

Samples Issue. The main disadvantage of path tracing is that it in inherently a noisy process, which converges rather slowly to the right result. The noise in Monte Carlo integration corresponding to the estimation error due to considering only a finite number of samples, which leads to integral values that can be too high or too low (or pixels that are too dark or too bright in the rendering image). The noise can be reduced by increasing the sample budget per pixel and vanishes within the limit, as the 
amount of samples increases to infinity, but the noise reduction is not linear with respect to the sampling rate. Actually, to reduce the magnitude of noise by a factor of two, we need to increase the sampling rate by a factor of four. This leads to rapidly increasing sampling budgets for non trivial scenes. Yet, the computation cost of path tracing is still problematic for offline rendering, and prohibitive for interactive or real-time rendering.

\subsection{Related Work}

In this section, the existing methods of reconstruction and denoising based previsualization for path tracing will be discussed.

\subsubsection{Reconstruction Techniques for Path Tracing}

Reconstruction techniques like compressive rendering [9] and compressive image reconstruction [23] have been proposed to address efficient previsualization of path tracing. As described in previous section, artifacts shown in intermediate previsualiations of path tracing are mainly due to improper or limited number of evaluated samples. Therefore, it is reasonable to formulate the issue as a data reconstruction problem to estimate missing samples (or pixels) using evaluated samples. The existing reconstruction based works can be divided into three branches: 1) inpainting based reconstruction; 2) compressed sensing based reconstruction; 3) matrix completion and separation based reconstruction.

\section{Inpainting based Reconstruction}

Image inpainting is widely discussed in various fields such as computer graphics, computer vision and image processing. The basic task of inpainting targets prediction of incomplete image pixels. The missing pixels include scratches, damaged photo stains and small holes. Inpainting is a 
positive research field, wherein the methods can be separated into semiautomatic inpainting methods, partial derivative equations (PDE) based algorithms, algorithms that are based on templates/models, texture synthesis methods, and hybrid technologies based on specific features. According to the PDE model, the first approach was proposed by Bertalmio et al. [24], wherein the information is spread in wide areas, via isophote lines across the edges. The algorithm has high efficiency for images which suffer narrow damage, because it applies anisotropic diffusion leading to reduction in blurring effects. The major disadvantage of the method is embodied in the fact that it cannot set up textures [25]. The methods developed by Taschler [2] belong to the same type. The authors have come up with an algorithm according to partial differential equations of the second order with use of diffusion and the previous improved one [26]. The significant problem was not solved, namely, the algorithm could not set up the textures. Tschumperle and Deriche [27] presented a method with application of partial differential equations of higher order. It is not an image restoration technique, but it generates good results for images which have occluded regions of small area and narrow results.

As for types of the semiautomatic inpainting methods, Sun et al. [28] came up with a technique that needs two steps for restoration. First, the user must describe contours of the object located in the occluded region, which begins from the outside to the inside, and then use texture synthesis that applies blocks or pixel images as a texture source. The method developed by Oliviera et al. [29] applies isotropic diffusion, which could maintain the contours and separate the edges from the mask. Hence, they do not suffer influences of smoothing. Telea [30] tried to make the improvement based on the estimated pixel value, which is restored by pixel neighborhoods only once for each pixel. In comparison with the iterative methods, a seam carving method was shown in [31], which addressed the efficiency issue.

With regard to the texture synthesis methods, the technique developed 
by Efros and Leung [32] takes a pixel as a starting point, while it is set on the edge of the occluded area and selects a window around it, so similar blocks in the region can be found. This method restores the texture for each pixel; therefore, the proposed algorithm overcomes the limitations of Bertalmios algorithm [24].

Heeger and Bergen [33] presented a texture reconstruction method with application of intermediate images collections that establish an image pyramid. Their method comprises the interaction, where the image pyramid is created through division of the damaged image and the one which embodies the source. Their method can obtain satisfactory texture results through repeating the iterative steps, but is only valid with regard to the stochastic types. Bonet [34] proposed an improvement to reproduce the regular textures by considering dependencies among different levels of texture granularity. Igehy and Pereira [35] discussed another version of the algorithm that was proposed by Heeger and Bergen. The method involved a new step with a mask containing subunit values and the information from the original image involved in texture synthesis. The similar techniques could involve an algorithm based on templates, which was proposed by Criminisi et al. [36]. The authors are describing a technique that emphasizes the order of the restored pixels. The algorithm starts from the edge of the occluded area, while each pixel from the edge is assigned a priority. Texture synthesis is completed with blocks by reusing the information from a source area, which is based on the priority value determined for each pixel.

The algorithm proposed by Drori et al. [37] involves specific granularity levels, which are used for estimating the best levels. It then sets a filling order with means of a confidence value, followed by the step of search similar to Efros and Leung. Their algorithm applies several different orientations of the block. The inpainting algorithm of Guillemot et al. [38] tries to find k-nearest neighbors concerning the damage to be filled. Then the k-nearest neighbor search is improved with linear regres- 
sion. Hays and Efros [39] came up with an approach which adopts a big image collection and uses it as a restoration database. As pointed out, the possibility in region restoring in a natural manner increases in view of the information quantity contained in the large images set. The restoration process includes checking of each item in the database aiming at possible matching of the damaged region with application of an image descriptor. The same approach was embodied by Le Meur and Guillemot [40], which provides an exemplar based inpainting framework. A coarse version is inpainted at first, so the computational complexity and noise sensitivity can be reduced, while the dominant orientations of image structures can be extracted. A novel sparsity concept aiming at the level of patch is introduced by Sun and $\mathrm{Xu}$ [41], so that the priority and representation of a patch can be modeled into two core processes for propagation in exemplar based method. Furthermore, Ladjal et al. [42] proposed experimental proof with regard to the exemplar based methods could establish the local geometric information. After implementation of minimization to variational models, geometry and especially smooth edges can be reconstructed.

One of the methods for hybrid inpainting was proposed by Bertalmio et al. [43], who developed an algorithm by dividing the original image into two layers. One of them involves the characteristics of structure while the second one includes the texture. The first image can be processed with a structural inpainting algorithm [24] and the second one can be processed with the texture synthesis algorithm that was put forward by Efros and Leung in [44]. The results generated from both operations lead to the final image. Atzori and de Natale [45] put forward another hybrid method. The restoration process starts from matching of the contours that runs through the edge of the occluded area. This operation will generate smaller regions which can be filled through copying of blocks from the outside. As proposed by Rares et al. [46], the global and local information should be treated as integration of edge contours which crosses the damaged area. Thus, more accurate pairs of lines can be achieved, but the matching pro- 
cess will be more complex. Recovered pixel values are then assigned based on the pixels near the new contours in the occluded area.

State of the art inpainting methods can reasonably recover missing pixels but often suffer from blurry artifacts in high-frequency areas. Recently, Li et al. [4] proposed a method to combine numerical and example based methods to address the issue. Although it requires a long recovery time, it shows promising results for recovering missing pixels in detailed regions.

\section{Compressed Sensing based Reconstruction}

Compressed sensing (CS) has been applied in the signal processing community to recover a signal when it satisfies sparse property under a transform domain. Specifically, it indicates that any sparse signal (like visual data transformed with a suitable basis) can be established with high quality based on a few measurements. That means existing rendering techniques can be accelerated through measuring or computing only a small number of samples, and the data can be accurately estimated by the CS method.

The performance exerted by a CS method is generally decided by the selection of a suitable basis, which can represent a signal. Since an image shows various sparsity patterns on different basis, to minimize the reconstruction error, the signal's representation must be as sparse as possible [47]. Most previous CS based image synthesis algorithms depend on the analytical basis, such as wavelets bases [9]. For this purpose, Sen et al. [9] only rendered a subset of pixels in image domain and applied a greedy, simple CS based algorithm for estimating the wavelet transformation of the image after rendering. Since the image's energy in the wavelet domain is concentrated more compactly, only a small set of samples is needed to obtain a satisfied result in comparison with the conventional spatialdomain rendering. However, the natural images deliver diverse features and information, a fixed analytical basis cannot get a sparse enough rep- 
resentation with the purpose of an accurate signal recovery. Hence, the reconstruction quality is poor and artifacts like speckle noise, ringing or blurring can be noticed.

Miandji et al. [23] presented a CS based algorithm with a learning based dictionary, which can accurately reconstruct incomplete visual data, including light fields, animations and images. The learning based approach allows the dictionary exploiting the sparsity patterns involving the visual data, and obtains a more sparse representation than the fixed analytical dictionaries. This is in comparison with the previous work [48], in which a single 1D-learned dictionary was used to apply CS for light fields. Miandji conducted training of 2D dictionary to represent the locally sparse data, which allows sparse representation in each cluster. Their results proved that signals can be embodied with the reduced union-of-subspaces model of signals [49], and the sparse representation can be admitted with a minimal size of dictionary. The key point of their method is that correlations in 2D can be explored by the dictionary. This allows the optimal recovery and requires a very small footprint of memory. The ensemble can be trained only once with regard to different signal types (light fields, images and videos).

Compressed sensing based methods generate the believable reconstruction of missing pixels in a partially rendered image. But it is limited in the case of low sampling rate, and the final output quality depends on selecting a suitable basis in transform domain.

\section{Matrix Completion and Separation based Reconstruction}

Matrix completion methods have been recently introduced in image processing to recover missing data in a low-rank matrix [16, 17]. Although solving the rank minimization problem is NP-hard, it can be approximated, and exactly solved in some useful cases, as a nuclear norm minimization (NNM) problem. Cai et al. provided a singular value threshold- 
ing algorithm (SVT) for solving NNM [50]. They demonstrated that the low-rank matrix completion problem could be solved by a soft thresholding operation on the singular values of the observed matrix.

Matrix completion and separation schemes have not been adopted in computer graphics until recently and we could find only two previous works. Recently, Hou et al. [22] took advantage of the low-rank property of a many lights matrix for solving many lights rendering problems using a matrix separation scheme. Li et al. [4] adapted matrix completion for inpainting while exploring a low rank matrix by grouping similar patches within an image. In the framework, they begin by searching and grouping similar patches with regard to the input image, and then estimating the missing pixels of the grouped patches by using matrix completion technique. The results are finally obtained by synthesizing all reconstructed patches. The main limitations of their method involve the accuracy of the patch matching and low-rank matrix completion. For the first issue, a method concerning robust patch matching was proposed. With regard to the second task, they applied the multipliers with alternating directions technique. Although they tested their inpainting scheme to recover missing pixels of a rendered image, their main focus was inpainting to recover missing pixels in photos, so they did not formulate sub-pixel and multiframe samples as in this thesis. Therefore, the proposed reconstruction based work in this thesis is unique in addressing the screen space recovery of incomplete path-tracing images using matrix completion across low rank matrices of sub-pixel, pixel, and multi-frame samples.

\subsubsection{Denoising Techniques for Path Tracing}

Despite the above-discussed reconstruction based method, another category of the solutions is the denoising based method. Extensive works have been proposed to address path tracing noise. Denoising techniques render an image with a few samples per pixel, and then remove the noise 
with a post-filtering operation. Some approaches also adaptively sample to further improve the results. Previous denoising based techniques can be divided into two general branches: 1) color based denoising filters that utilize sample color value for filtering, and 2) scene feature based denoising filters that utilize additional scene features.

\section{Color Based Denoising Filters}

Color based denoising filters for path tracing are often enlightened with traditional image denoising methods and solely utilize the rendered pixel color to remove path tracing noise. Redner and Lee [51] proposed a seminal work which defines alpha-trimmed filter based on the median/mean filter. Christensen and Jensen [52] applied a median filter or Gaussian filter within a local area, which the light has been reflected at least two times. The trilateral filter proposed by Choudhury [53] concerns the image gradient and an adaptive neighborhood function. Another classic bilateral filter was developed by Pattanaik and $\mathrm{Xu}$ [54] which is different from the work proposed by Redner and Lee [51], as classical bilateral filters will not remove the outliers. To address this limitation, the bilateral filter weights proposed by Pattanaik and $\mathrm{Xu}$ are calculated via the denoised image. Bala et al. [55] presented an efficient filter with an edge image. In addition, Egan et al. [56] applied frequency analysis for shearing of filter and distributed effects like motion blur and occlusion or shadowing. Mehta et al. [57] applied correlated analysis to generate simple formulas that use variance of Gaussian filter of the screen space to remove noise from specific rendering effects.

More complex filters apply sample information based on the renderer for filtering on the pixel level. Ward and Rushmeier [58] set an influence region of noisy pixel based on comparing whether the variance of noisy pixel is smaller than a certain threshold after several iterations. Each noisy sample can make a contribution to other denoised pixels, and no energy 
will be leaked since the filter is normalized. McCool [59] introduced another classical filter by applying geometric information of pixels. It is an anisotropic diffusion that removes the path tracing noise. Normal and depth information collected in rendering (included in G-buffer) and the color coherence map are used, while the conductivity function of the diffusion scheme in the pixel is estimated from the coherence map. More recently Tschumperle et al. [10] proposed a scheme with an efficient wavelet filter for global illumination of path tracing. Hence, the filter adopts information of the buffer concerning indirect or direct illumination, as well as the scene feature buffer based on point position or vector normals. Additionally, $\mathrm{Xu}$ et al. [60] invoked the bilateral filter for denoising images generated with light paths, which are complicated in fog or smoke. According to the path gradient direction, in their paper, additional bilateral weights are used for better guidance of the denoising scheme. Most of these last approaches also adaptively position samples with the analysis.

In order to remove noise of the general rendering effects, a shrinkage of wavelets is adapted to path tracing noise reduction by Overbeck et al. [61]. Rousselle et al. [62] chose an appropriate scale for the Gaussian filter on each pixel to reduce the recovery error. In addition, they made improvements of it by using the non-local means filter [63]. Since the path tracing noise at every pixel can be estimated with the absolute median deviation. Sen and Kalantari [64] can use the arbitrary image denoising method for path tracing. Lastly, Delbracio et al. [2] put forward a method subject to non-local means filtering, which predicted the distance between the selected patches together with the color histograms. The color based methods can be easily adapted to handle different distributed effects, but they usually request many samples to obtain satisfy results. For challenging scenes, they may fail to generated reasonable results at low sampling budget. 


\section{Scene Feature Based Denoising Filters}

With this type of methods, additional feature buffers (such as texture values, shading normals and world positions) calculated with the render can be leveraged. Thus, they can generate higher-quality results in comparison with the color based methods in previous section. For efficiently rendering global illumination of a scene, Laine et al. [65] and Segovia et al. [66] applied the geometry buffer. For removing the global illumination noise of path tracing, Dammertz et al. [10] integrated the wavelet information and bilateral filter, while Bauszat et al. [67] applied a filter with the guided image. In addition, a G-buffer was applied by Shirley et al. [68] to deal with depth-of-field and motion blur effects, and Huo et al. [69] made combination of a G-buffer and variance for filtering the noise from depth-of-field. These scene feature based approaches only concern several rendering effects which are not general. For removing different path tracing noise with auxiliary features from render, Sen and Darabi [15] demonstrated the benefit of adaptively setting the filters feature weights through the image. They made computation with these weights and mutual information, so they can approach the functional dependencies existing in random parameters and scene features. Li et al. [1] applied a cross-bilateral filter with Steins unbiased risk estimator (SURE) [70] for estimating the proper spatial filter parameter, and the cross terms weights is conducted by hard coding. Rousselle et al. [71] made improvement on this with the SURE metric for selection among three candidates across the non-local mean filters, and weight features and colors differently.

Darabi and Sen [72] applies all information concerning rendering and each numerical photon for denoising with use of bilateral filtering. In addition, the bilateral filter also considers the spatial neighborhood and the sample position, the scene features (texture values, world space position and normal), the random synthesis parameters, and the sample color finally. The computation of mutual information concerns dependence of statistics involving randomly generate filter parameters at the position 
that share identical colors or features. They achieved outstanding results based on a low sample budget per pixel, but the high computation of the method means it is not suitable for previsualization. In addition, Lehtinen et al. [73] introduced a denoising method with the anisotropy property of temporal light field, which can handle various effects like depth-offield, motion blur and soft shadows. Specifically, the effective sampling rate increases by a big factor subject to efficient reuse of pixel samples. Recently Lehtinen et al. [74] conducted generalization of these ideas for the indirect illumination. Through contemplating of the diffuse surface properties, their algorithm can interpolate the light field for the denoised results, which is as similar as the image rendered with many samples. For instance, for the input path-tracing image rendered with 8 samples per pixel, the denoised result of this input have similar visual quality with the reference image that rendered with 512 samples per pixel. The main problem of scene feature based methods is that the weight of every filter term is estimated according to error metric and/or heuristic rules which will contain noise at low sampling rates. Therefore, they cannot predict proper filter weights in challenging cases.

\section{Deep Learning Based Denoising Filters}

Recently, convolutional neural network (CNN) has appeared as a ubiquitous model in machine learning, which achieved state-of-the-art performance in various tasks such as speech processing [75] and image classification [76]. CNNs have also been widely applied with regard to various image-processing, low-level tasks. Particularly, several works have taken into account the natural image denoising $[77,78]$ and image superresolution [79] which is highly correlated.

Unlike the aforementioned techniques, deep learning based path tracing denoising methods learn the complex relationship between noisy rendering and reference data by leveraging both the color values and the aux- 
iliary scene features. Our proposed denoising based method in Chapter 5 is also inspired by the recent deep learning based denoising techniques. Kalantari et al.[80] firstly applied a plain neural network to predict parameters for a cross-bilateral or non-local filter. In their method, a complicated relation between the noisy scene data and the ideal filter parameters is observed, while this relationship is learned with a nonlinear regression model. For this purpose, they used a perceptron neural network with multiple layers and made combination with a matching filter during testing and training. The network is first trained offline with noisy images including various distributed effects. Then, during running, the trained network can estimate the filter parameters on different rendering to generate the denoised results that close to the reference rendering.

Later, Bako et al.[81] improved this approach by using a kernel prediction network to compute the filtered pixel value from its local neighbors. They put forward a supervised learning method which enables the filtering kernel become more complicated and general by applying a deep convolutional neural network (CNN) architecture. As one embodiment of their framework, the CNN makes direct prediction of the denoised pixel value through a non-linear representation of the rendered feature buffers. As for the second approach, they applied a kernel-prediction network that adopts the CNN for estimation of the local weighting kernels for computing each denoised pixel from the surrounding neighbors. Recently, Vogels et al.[82] extended the concept over a temporal window predicting kernels for an entire sequence of subsequent animation frames. Chaitanya et al.[83] propose a convolutional architecture which enforces temporal stability via recurrent connections that provide a mechanism to incorporate information from past frames in a sequence. But under a low sampling budget, these methods may generate over blurred results and fail to provide accurate previsualization. 


\section{Chapter 3}

\section{Matrix Completion for Path Tracing}

As discussed in Chapter 1, path tracing is well established for photo-realistic rendering to simulate light path transport. The method has been adapted in many applications to produce high quality synthetic images requiring a huge number of samples and long computation time. Due to the high cost to produce the final output, intermediate previsualization of path tracing is in high demand from production artists to detect errors in the early stage of rendering.

Visualizing intermediate results of path tracing is challenging since the synthesized image with limited samples or improper sampling usually suffers from distracting noise. The conventional practice is to render the frames at a lower resolution and upsample them for previsualization. However, this has clear drawbacks due to the limitation in visual quality as well as a waste of resources: we cannot reuse the low-resolution outputs for final rendering. The ideal solution would be to provide a highly plausible intermediate result in the early stages of rendering, using a small fraction of samples, and also adapt additional samples in a progressive manner to minimize any unnecessary rendering. Furthermore, if the previsu- 
alization relies only on samples, and not on scene information, it can augment various rendering algorithms as an additional postprocessing step. In this chapter, we present a screen space reconstruction that addresses these challenges.

In this chapter, we present a novel reconstruction method using matrix completion that efficiently recovers missing data in incompletely rendered images, which is randomly and sparsely rendered under fixed sampling rate (e.g. $40 \%$ ). The fundamental task of matrix completion is to estimate missing entries in a matrix. Recently this problem has been successfully solved by making use of the assumption that the matrix has lowrank property. This assumption requires that the reconstructed signal be as simple as possible in a certain sense, and plays a similar role to the generic smoothness assumption that is often employed in inpainting and other problems. However, unlike the smoothness assumption, the lowrank assumption allows edges in certain cases. For example, an $n \times n$ matrix $M_{i, j}:=\operatorname{sign}(j-n / 2)$ has exactly one non-zero singular value.

Applied to our problem, the basic strategy is to construct a matrix from a group of similar pixels or patches, and then use matrix completion to estimate the missing samples. The availability of surrounding, similar pixels or patches helps guide the recovery.

We approach the low-rank matrix construction step differently for pixel samples and for sub-pixel samples (a fraction of samples within a pixel) of images, and for multi-frame samples in animation sequences.

For pixel samples, we form a pixel patch using neighboring pixels, and then group similar pixel patches from elsewhere in the image to form a low-rank matrix [4]. However, identifying similar pixel patches across an image has extra challenges due to missing pixel values. A sophisticated pre-completion step is required to estimate missing pixel values in each patch [4]. In this chapter, we use a pre-trained convolutional neural network $(\mathrm{CNN})$ to precompute the missing values efficiently in a short time.

For multi-frame samples, the intuitive assumption to form a low-rank 
matrix is to use redundancy across nearby frames. We therefore adapt a similar approach, gathering similar patches from nearby frames rather than from within a single image. The scheme has been applied to missing pixel recovery in multiple adjacent frames and efficiently reduces temporal noise in a video.

For sub-pixel samples, we apply the same general idea, but rather than using similar patches in the image or adjacent frames; we draw samples from adjacent pixels (Figure 3.5). Considering the approximate selfsimilarity of image statistics, it would seem that the same strategy could be used at both pixel and sub-pixel levels. For example, an edge with missing pixels is easier to identify when more of the edge is present, even if the ratio of known to missing pixels remains constant (Figure 3.6). Our choice of differing strategies can be motivated in terms of a speed/quality trade-off. The expected variance within a region grows as the region size increases, so simply choosing adjacent pixels is less likely to be successful at larger scales. On the other hand, using local information is cheaper than searching elsewhere in the image, and exact recovery is less likely to be crucial at sub-pixel scales, so we make use of only local information in this case.

Finally, the missing values of a low-rank matrix formed from either missing pixel, sub-pixel, or multi-frame samples are recovered by our novel matrix completion step. For efficient recovery of high-frequency images, we adapt a multi-weighted nuclear norm minimisation (WNNM) scheme that optimizes both high and low singular values differently. Then, we added a novel progressive parameter (PA) adjustment strategy to WNNM (PAWNNM). Our PAWNNM reduces the WNNM weights during each iteration of the optimization, resulting in a progressive quality increase.

This chapter is started by introducing the background of matrix completion (Section 3.1). To utilizing matrix completion, we need to show how we generate the related low matrix in different scenarios (Section 3.2). Our optimized algorithm is described in Section 3.3. Furthermore, we compare 
our results with other reference works and demonstrate that our technique is more efficient and optimal.

\subsection{Overview}

Matrix completion has been recently introduced $[17,16]$ and successfully applied to many problems in image processing [20,21], but has not been adopted in the computer graphics community until recently; we found only one recent paper, which addresses many lights rendering [22]. We first make a fundamental review of the standard matrix completion method.

The values of a sparsely and randomly sampled matrix $M$ can be approximated by matrix completion if $M$ has low-rank [17]. Then, the problem can be formulated as a low-rank matrix minimization:

$$
\begin{array}{ll}
\min _{X} & \operatorname{rank}(X) \\
\text { s.t. } & X_{i j}=M_{i j}, \quad(i, j) \in \Omega
\end{array}
$$

where $X$ is an estimate to be solved by rank minimization constraint by observed samples, and $\Omega$ contains the positions of the observed samples.

Although solving the rank minimization problem is NP-hard, Candes and Recht showed this problem has a convex relaxation as a nuclear norm minimization (NNM) problem [17]:

$$
\begin{array}{ll}
\min _{X} & \|X\|_{*} \\
\text { s.t. } & X_{i j}=M_{i j}, \quad(i, j) \in \Omega
\end{array}
$$

where the nuclear norm $\|X\|_{*}$ is defined as $\|X\|_{*}=\sum_{s=1}^{n} \Sigma_{s}(X)$, and $\Sigma_{s}(X)$ are singular values of matrix $X$.

In our method, we use singular value thresholding (SVT) algorithm for solving the NNM. SVT iteratively applies a soft thresholding operation to the singular values of $X$ [50]. The solution to the SVT formulation in Equation (3.3) converges to Equation (3.2) for large threshold $\lambda$. 


$$
\begin{array}{cl}
\min _{X} & \frac{1}{2}\|X-M\|_{F}^{2}+\lambda\|X\|_{*} \\
\text { s.t. } & P_{\Omega}(X)=P_{\Omega}(M)
\end{array}
$$

where \|\|$_{F}^{2}$ is the Frobenius norm, and $P_{\Omega}$ is the orthogonal projector onto the span of matrices vanishing outside of $\Omega$, so that the $(i, j)$ component of $P_{\Omega}(X)$ is equal to $X_{i j}$ for $(i, j) \in \Omega$ and zero otherwise.

It can be solved by iterating Equation (3.4) from an initial matrix $Y_{0}=0$ with a given $\lambda>0$ until a stopping criterion is reached. It has been shown that the sequence $X^{k}$ obtained via Equation (3.4) exactly converges to the unique solution of Equation (3.3) [17].

In Equation (3.4), $D_{\lambda}$ is the singular value shrinkage operator on each singular value $\Sigma_{i}$ of $Y: D_{\lambda}(Y)=U S_{\lambda}(\Sigma) V^{T}$, where $S_{\lambda}(\Sigma)=\operatorname{diag}\left(\max \left(\Sigma_{i}-\right.\right.$ $\lambda, 0)$ ), and $\sigma_{k}$ is a positive step size sequence.

$$
\left\{\begin{array}{l}
X^{k}=D_{\lambda}\left(Y^{k-1}\right) \\
Y^{k}=Y^{k-1}+\sigma_{k} P_{\Omega}\left(M-X^{k}\right)
\end{array}\right.
$$

The Lagrangian for problem (3.3) is given by

$$
L(X, Y)=\lambda\|X\|_{*}+\frac{1}{2}\|X\|_{F}^{2}+\left\langle Y, P_{\Omega}(M-X)\right\rangle
$$

where $\mathrm{Y}$ is the Lagrangian dual variable. Cai et al. show that SVT indeed optimizes the dual function $f(Y)=\inf _{X} L(X, Y)$ via the gradient descent method[50]. The SVT algorithm shows great efficiency for solving the matrix completion task, especially for a large low-rank matrix. Besides matrix completion, it has been widely applied to solving other nuclear norm minimization based problems, such as robust principle component analysis $[16,84]$, texture rectification and denoising [85] and low-rank representation for subspace clustering[86]. 


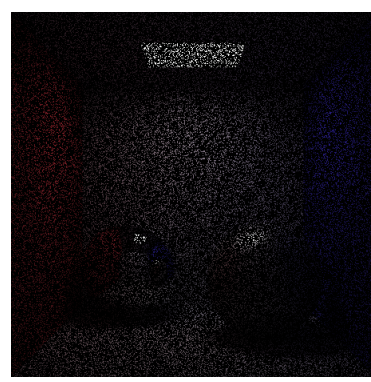

Input (20\% pixels)

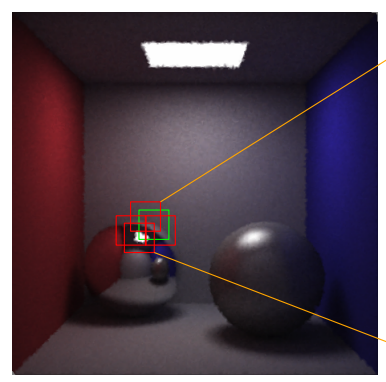

pre-completed image via $\mathrm{CNN}$

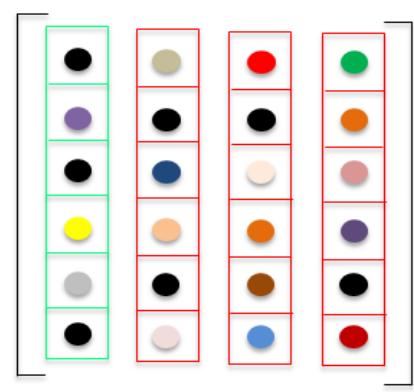

low rank matrix $M_{p}$

Figure 3.1: Low-rank matrix construction for pixel patch: from the left, the input image (20\% pixel samples), pre-completion and grouping, and low-rank matrix $M_{p}$. From the initialized image (middle), similar pixel patches (red squares) for a current pixel patch (green square) are selected, and stored into columns of the matrix $M_{p}$.

\subsection{Low-Rank Matrix Construction}

In order to estimate missing values in an incomplete path tracing image using matrix completion, we need to provide a proper low-rank matrix that contains sparse random samples evaluated by path tracing rendering. We present three different schemes for constructing low-rank matrices in different scenarios such as an image containing a fraction of missing pixels, missing pixels in multi-frames, and a fraction of missing sub-pixels within a pixel. Accordingly, the constructed low-rank matrix in the three scenarios is as follows.

Pixel Patch: We recover missing pixels in an incompletely rendered image (Figure 3.1) using matrix completion. We pre-complete the missing pixels using a convolutional neural network $(\mathrm{CNN})$. A set of similar pixel patches (Figure 3.1, middle) are computed to form a low-rank representation across the whole image space. The similar pixel patches are fed into column vectors of a matrix $M_{p}$ (Figure 3.1). The precompleted missing values of $M_{p}$ are recovered by matrix completion (see Section 3.3). The details 
of the construction process of $M_{p}$ are discussed in Section 3.2.1.

Multi-frame Patch: Pixels in adjacent frames have a high chance to have similar values, and therefore similar values across frames provide a low-rank form. Similar pixel patches of adjacent frames are computed and fed into a matrix $M_{m}$ as in Figure 3.4. The missing pixels in adjacent frames are simultaneously recovered by matrix completion.

Sub-pixel Patch: A group of randomly selected samples within a tiny area such as within a pixel have a high chance to be similar. The proposed idea is to form a low-rank matrix that contains a fraction of sub-pixels within a pixel and estimates the missing sub-pixels using low-rank matrix recovery. A fraction of a sub-pixel grid is evaluated by path tracing. The surrounding sub-pixel grids are grouped to form a sub-pixel patch and fed into a low-rank matrix $M_{s}$. The details are discussed in Section 3.3.3.

\subsubsection{Pixel Patch}

In CR [9], missing pixels in an incomplete image $I_{m}$ are estimated by compressed sensing: $I_{m}$ consists of a portion of randomly selected and rendered pixel samples as well as missing pixel samples. We address the problem using a matrix completion scheme while constructing multiple low-rank matrices, $M_{p} \mathrm{~s}$ consists of similar pixel patches, $\widetilde{P}_{p} \mathrm{~s}$ within an image. The idea is inspired by a non-local image processing scheme [87], [63] such that a group of mutually similar patches, $\widetilde{P}_{p}$ s provides low-rank property [4].

Pre-completion using CNN: Computing similar pixel patches is technically challenging due to many missing pixels. Li et al. [4] proposed a pre-completion step that initializes missing pixels [4]. They address pre-completion for low frequency and high-frequency images differently. For a low frequency image, matrix completion is directly applied for precompletion, and a total variation (TV) regularized reconstruction [88] is used for high-frequency images that do not meet the low-rank condition. 
Although it provides promising initialization, the process requires long computation time that is a clear limitation to adapting in the method for previsualization.

We present a novel pre-completion scheme using a convolutional neural network $(\mathrm{CNN})$. A CNN is trained by pairs of patches from a reference image $I_{r}$ and an incomplete image $I_{y}$ that contains randomly removed pixel values in $I_{r}$. Then, the pre-trained $\mathrm{CNN}$ is used to initialise missing values for pre-completion. For our neural network, a loss function $L(\Theta)$ is defined to measure error between the estimated data $F(y ; \Theta)$ that contains patches of $I_{y}$ and the reference data $R$ that contains patches of $I_{r}$ :

$$
L(\Theta)=\frac{1}{n} \sum_{i=1}^{n}\left\|F\left(I_{y}^{i} ; \Theta\right)-R_{i}\right\|^{2}
$$

where $n$ is the number of training samples and $\Theta$ is a network parameter to learn. $F\left(y_{i}\right)$ is the pre-completion result defined by:

$$
F_{l}(y)=\hat{f}\left(W_{l} \otimes F_{l-1}\left(I_{y}\right)+B_{l}\right)
$$

where $l$ is the number of layers, $W_{l}$ are convolutional filters having different sizes $\left(s_{l} \times s_{l}\right)$ and numbers $\left(n_{l}\right)$ in each layer $l$, and $B_{l}$ are the biases, $\otimes$ is the convolution operator, and $\hat{f}$ is the sigmoid function: $\hat{f}(x)=1 /\left(1+e^{-x}\right)$. The network parameter $\Theta$ in Equation (3.6) is then defined as $\Theta=\left\{W_{i}, B_{i}\right\}$ [89], and $L(\Theta)$ is minimized by standard back propagation:

$$
\begin{array}{r}
\Delta_{i+1}=0.9 \times \Delta_{i}+\eta \times \frac{\partial L}{\partial W_{i}^{l}} \\
W_{i+1}^{l}=W_{i}^{l}+\Delta_{i+1}
\end{array}
$$

where $\Delta$ is momentum, $\eta$ denotes the learning rate, and $i$ is the iteration. The basic structure of our CNN is illustrated in Figure 3.2. The CNN is a three-layer network. The filter size $s_{l}$ and number of filter $n_{l}$ of $W_{l}$ in three layers are initialized as: $s_{1}=9, s_{2}=3, s_{3}=5, n_{1}=128$, and $n_{2}=64$. The weights of convolutional filter $W_{l}$ in Equation (3.7) are set by a Gaussian 


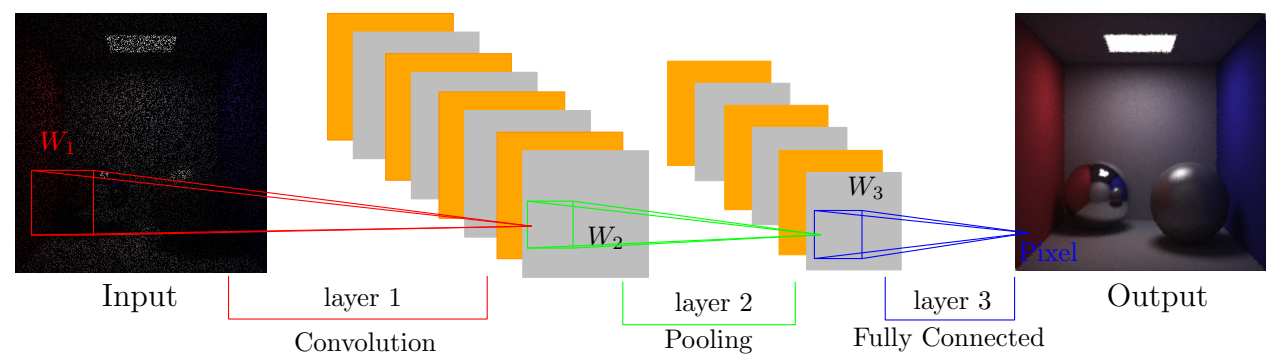

Figure 3.2: Three layered CNN architecture for pre-completion: the red square is a convolutional filter in layer $1\left(W_{1}\right)$, the green square is a convolutional filter in layer $2\left(W_{2}\right)$, and the blue square is a convolutional filter in layer $3\left(W_{3}\right)$.

distribution with zero mean with 0.001 standard deviation, and basis $B_{l}$ is initialized by zero. We use 130k patches from 500 images from Flickr (https://www.flickr.com/) for the training set with the learning rate $\eta=$ 0.00001 in Equation (3.8). The network is trained with different missing rate of input data.

Table 3.1: PSNR compared with CNN and TV initialization under $10 \%$ input pixels. The recovery time are measured in seconds.

\begin{tabular}{lcc}
\hline Input 10\% pixels & $\mathrm{CNN}$ & $\mathrm{TV}$ \\
\hline Toasters & 39.74 & 30.59 \\
Bottles & 30.17 & 19.73 \\
Bedroom & 31.82 & 23.91 \\
Bamboo & 26.03 & 23.01 \\
Recovery Time & $4 \mathrm{~s}$ & $90 \mathrm{~s}$ \\
\hline
\end{tabular}

In Table 3.1, we compare the PSNR (peak signal-to-noise ratio) of our $\mathrm{CNN}$ initialisation results with TV based initialisation, used in [4]. Even under a low sampling rate, our $\mathrm{CNN}$ based initialisation shows better re- 
sults than TV in shorter time. An image comparision of the toasters scene is in Figure 3.3. More comparison results can be found in Appendix A.

Patch matching and grouping: After pre-completion, we create a pixel patch $P_{p}(i, j)$ in pixel $(i, j)$ having $n_{p} \times m_{p}$ pixels (e.g., green square in Figure 3.1), and the similar pixel patches $\widetilde{P}_{p}$ s within the whole image space (e.g., red squares in Figure 3.1) are computed by weighted $L_{2}$ distance. In the weighted scheme, we assign more weights $(\mathrm{w}=0.75)$ for rendered pixels than initialized pixels from the pre-completion. The selected similar patches are stored in each column of a low-rank matrix $M_{m}$ as in Figure 3.1, and the missing values in the matrix are estimated by our low-rank matrix recovery step (see Section 3.3). After completing missing values in patch $P_{p}(i, j)$, the next patch $\left(P_{p}\left(i+\delta_{p}, j\right), 1 \leq \delta_{p}<\min \left(n_{p}, m_{p}\right) / 2\right)$ will be iteratively processed.

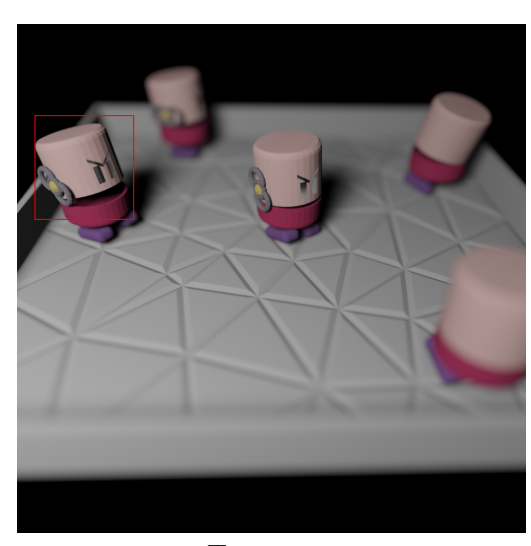

Toasters

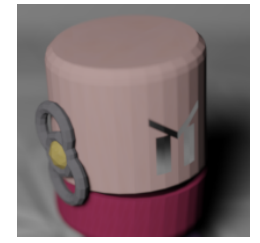

Reference

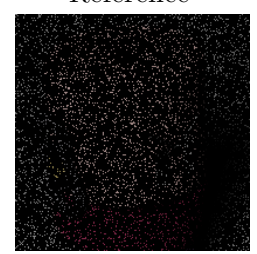

Input $10 \%$ pixels
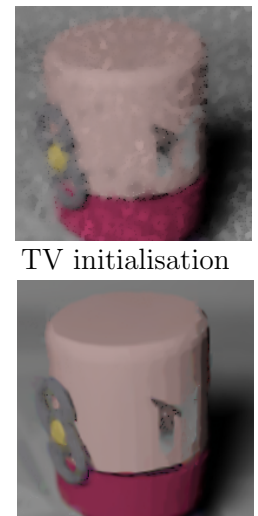

Recovery based on TV PSNR: 41.28 SSIM: 0.913

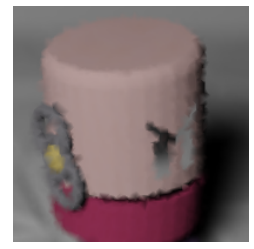

CNN initialisation

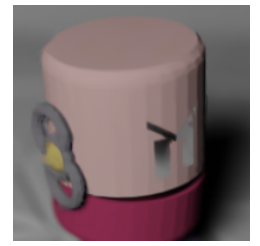

Recovery based on CNN

PSNR: 46.37 SSIM: 0.941

Figure 3.3: A visual quality comparison of initialisation between Total Variation and our CNN for a toasters scene, and the corresponding recoveries. Our recovery also achieve better recovery in terms of SSIM (the structural similarity) and PSNR. 


\subsubsection{Multi-frame Patch}

Path tracing is often used to produce multi-frame images for a video. Intuitively, a pixel $(i, j)$ in a frame $k, p_{k}(i, j)$ has a high chance to have similar values in adjacent pixels of nearby frames such as $p_{k-1}(i-1, j-1), \ldots$, $p_{k+1}(i+1, j+1)$. The similar values across frames provide low-rank property to form a matrix $M_{m}$ for matrix completion to estimate missing values in multiple frames simultaneously. Images of each frame are sparsely and randomly sampled as in the previous section. A pixel patch $P_{p}(i, j)$ of a pixel $(i, j)$ in frame $k, P_{p}^{k}(i, j)$ having $n_{p} \times m_{p}$ elements is created. Then, pixel patches $\widetilde{P}_{p, i}^{k},\left(0 \leq i \leq n_{f}\right)$ that are similar to $P_{p}^{k}$ are selected within the frame $k$ and grouped as $F_{k}=\left\{\widetilde{P}_{p, 0}^{k}, \ldots, \widetilde{P}_{p, n_{f}}^{k}\right\}$. Similar pixel patches in nearby frames are also selected and grouped (i.e., $F_{k-1}, \ldots, F_{k+1}$ ). Since each $F_{k}$ consists of a mixture of rendered and missing samples, the missing samples are initialized using a pre-trained $\mathrm{CNN}$, which is same CNN in pixel recovery. Then, a weighted $L_{2}$ norm is applied to measure the similarity across the initialized patches. Regions for searching similar patches are limited to the adjacent areas of the reference pixel $(i, j)$ across nearby frames. Similar pixel patches in each frame $F_{k}$ are assigned to columns of a low-rank matrix $M_{m}$. For example, similar pixel patches in 3 nearby frames $\left[F_{k-1}, F_{k}, F_{k+1}\right]$ can be assigned to $M_{m}$ as in Figure 3.4. Missing elements of pixel patches in multiple frames are estimated simultaneously in the subsequent matrix recovery step.

\subsubsection{Sub-pixel Patch}

Distracting artifacts in a path tracing image can be caused by the limited number of sub-pixel samples available for evaluating each pixel value. Therefore, we propose to address the problem as matrix completion that estimates missing sub-pixel samples required to correct each pixel value. The basic idea is to form a low-rank matrix that contains a fraction of the sub-pixel samples within a pixel and estimates the missing samples using 

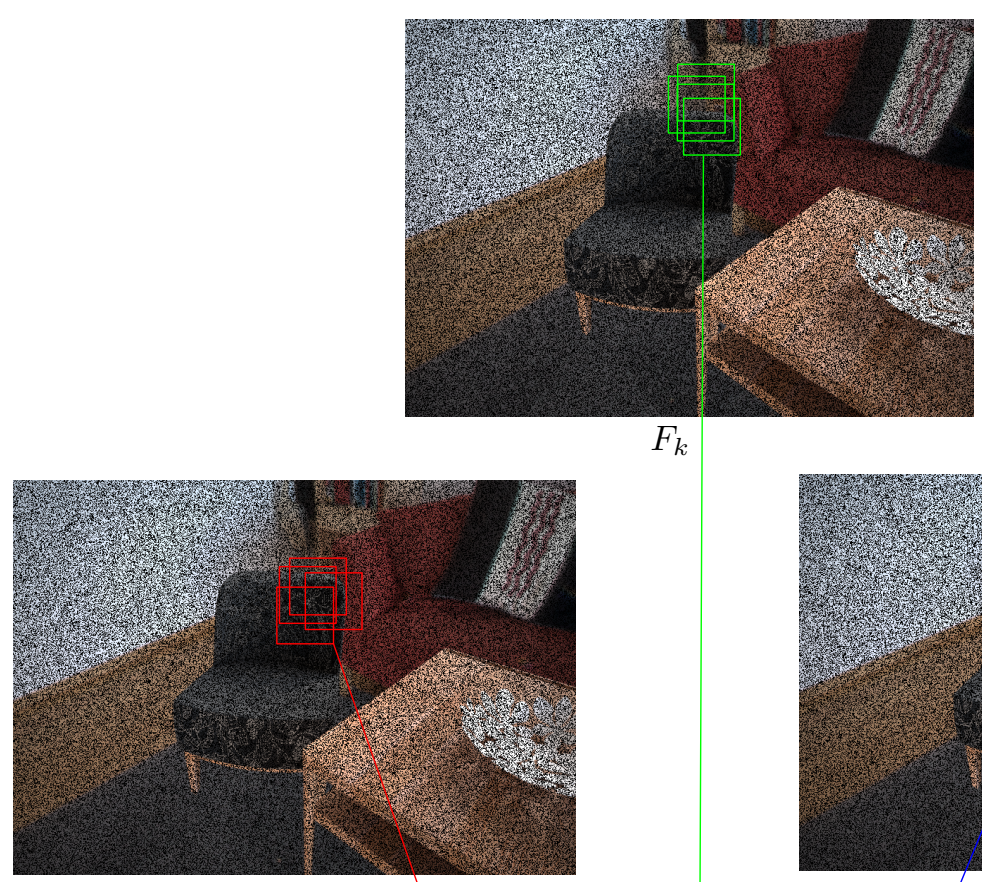

$F_{k}$
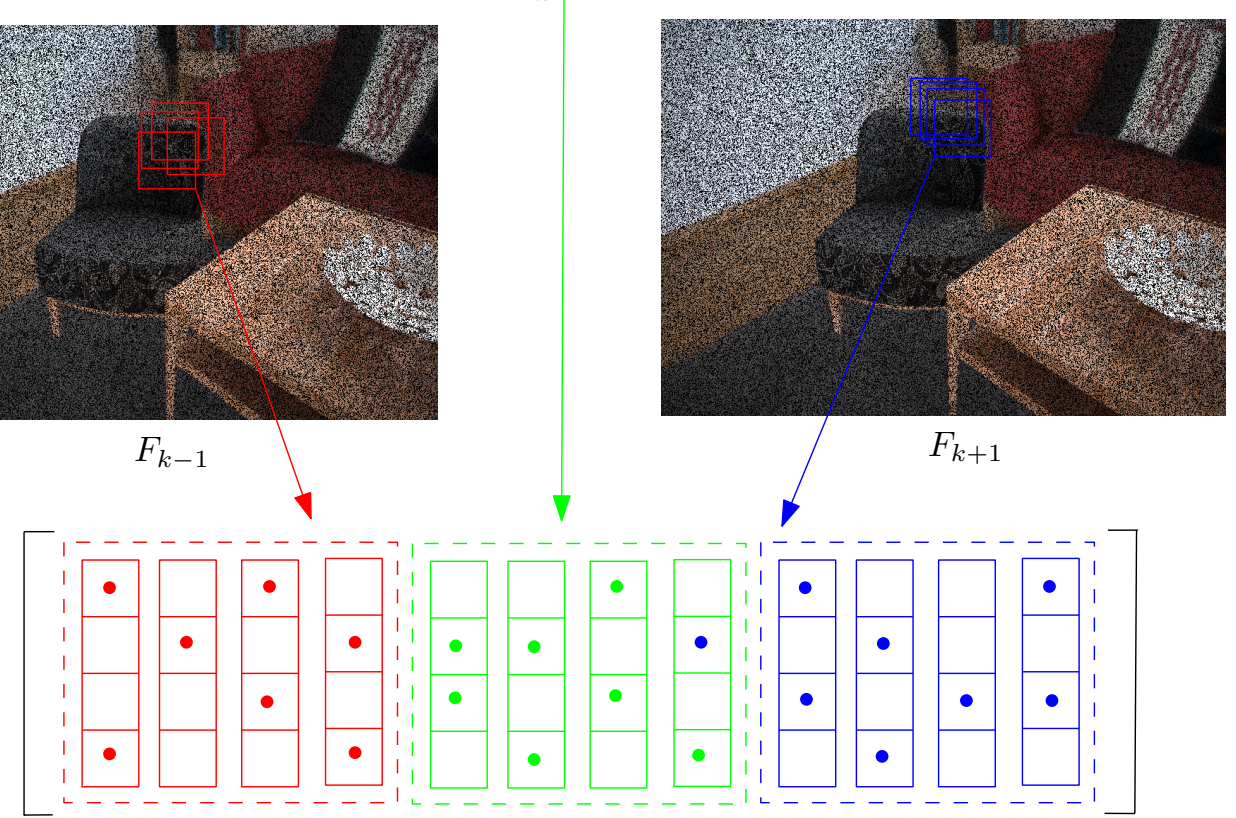

low rank matrix from multi-frame patches

Figure 3.4: An example of multi-frame patches $F_{k}$ and the constructed low-rank matrix $M_{m}$ : similar patches across multiple frames (squares) are stored in columns of a low-rank matrix. 
our low-rank matrix recovery method. We form sub-pixel patches $P_{s}$ as in Figure 3.5 to form a low-rank matrix $M_{s}$ for matrix completion. A uniform sub-pixel grid $G_{s}(i, j)$ having $n_{s} \times m_{s}$ elements is associated with a pixel $(\mathrm{i}, \mathrm{j})$ as in Figure 3.5 (left). A fraction of elements in $G_{s}$ are sparsely and randomly selected, and the values are evaluated by path tracing. In most cases of rendering, $G_{s}$ has the low-rank properties required for $M_{s}$, based on the fact that a group of randomly selected samples within a tiny area such as a pixel will have similar values within the local area. In some cases, such as an edge boundary, sub-pixel samples within a pixel might have high-frequency. In order to handle this case, we construct a sub-pixel patch $P_{s}(i, j)$ that consists of a group of sub-pixel grids surrounding the pixel $(i, j)$. One example of a $P_{s}$ having $3 \times 3$ elements is $P_{s}(i, j)=\left\{G_{s}(i-1, j-1), G_{s}(i-1, j), \ldots, G_{s}(i+1, j+1)\right\}$ as in Figure 3.5. The assumption behind our sub-pixel patch is that although a pixel value may be evaluated by high-frequency sub-pixel samples (e.g., edges), the surrounding pixels will have high probability to have low frequency subpixel samples (e.g., non-edge), and therefore the associated matrix can be a low-rank (Figure 3.6). Elements of each sub-pixel patch in $P_{s}$ are stored in a matrix $M_{s}$ and the missing elements of $M_{s}$ (the missing sub-pixel samples of $P_{s}$ ) are reconstructed in the following matrix recovery step (see Section 3.3). Our idea has been evaluated with various scenes including distribution noise (motion blur, soft shadow) as well as path tracing noise (see Section 3.4.4).

\subsection{Low-rank Matrix Recovery}

NNM: The low-rank matrix created in the three different scenarios is constructed using matrix completion [17]. In particular, missing values in the low-rank matrix are recovered using the SVT algorithm [50]. Here, $M$ denotes the incomplete low-rank matrix and $X$ is the original matrix we want to approximate. The nuclear norm minimization (NNM) problem is 


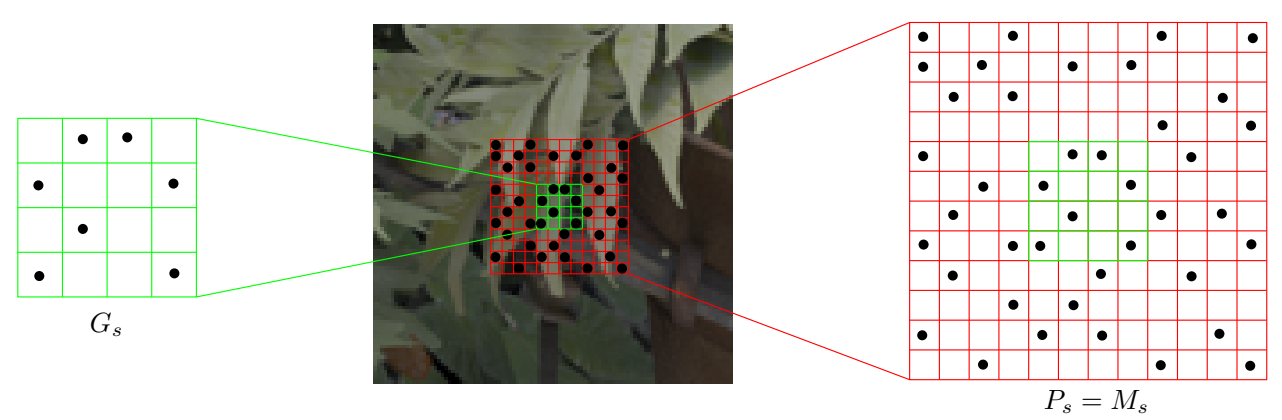

Figure 3.5: A sub-pixel grid $G_{s}$ (green) and $3 \times 3$ sub-pixel patch $P_{s}$ (red) fed into a low-rank matrix $M_{s}$ : black dots are rendered samples.

formulated in Equation (3.3) and the energy function can be defined as:

$$
\hat{X}=\arg \min _{X} \frac{1}{2}\|M-X\|_{F}^{2}+\lambda\|X\|_{*}
$$

where $\lambda$ is a positive weight, and $\hat{X}$ can be obtained by

$$
\hat{X}=U S_{\lambda}(\Sigma) V^{T}
$$

where $M=U \Sigma V^{T}$ is the SVD of $M$, and $S_{\lambda}(\Sigma)$ is the soft-thresholding function on the diagonal matrix $\Sigma$ with constant weight $\lambda$. For each diagonal element $\Sigma_{i i}$ in $\Sigma$, we set

$$
S_{\lambda}(\Sigma)_{i i}=\max \left(\Sigma_{i i}-\lambda, 0\right)
$$

WNNM: In a standard nuclear norm minimization, each singular value is treated equally and the soft thresholding operator shrinks each singular value with a constant $\lambda$. In order to optimize high-frequency details efficiently, we adapt the weighted nuclear norm minimization (WNNM) algorithm [90] to utilize the prior knowledge of singular values in the matrix. The main idea is that the larger singular values should be shrunk less to preserve the major data components. To adapt the WNNM, we modified the constant weight $\lambda$ in Equation (3.12) as multiple weights $\lambda_{i}$ :

$$
\lambda_{i}=\omega_{i} /\left(\Sigma_{i i}(X)+\epsilon\right)
$$




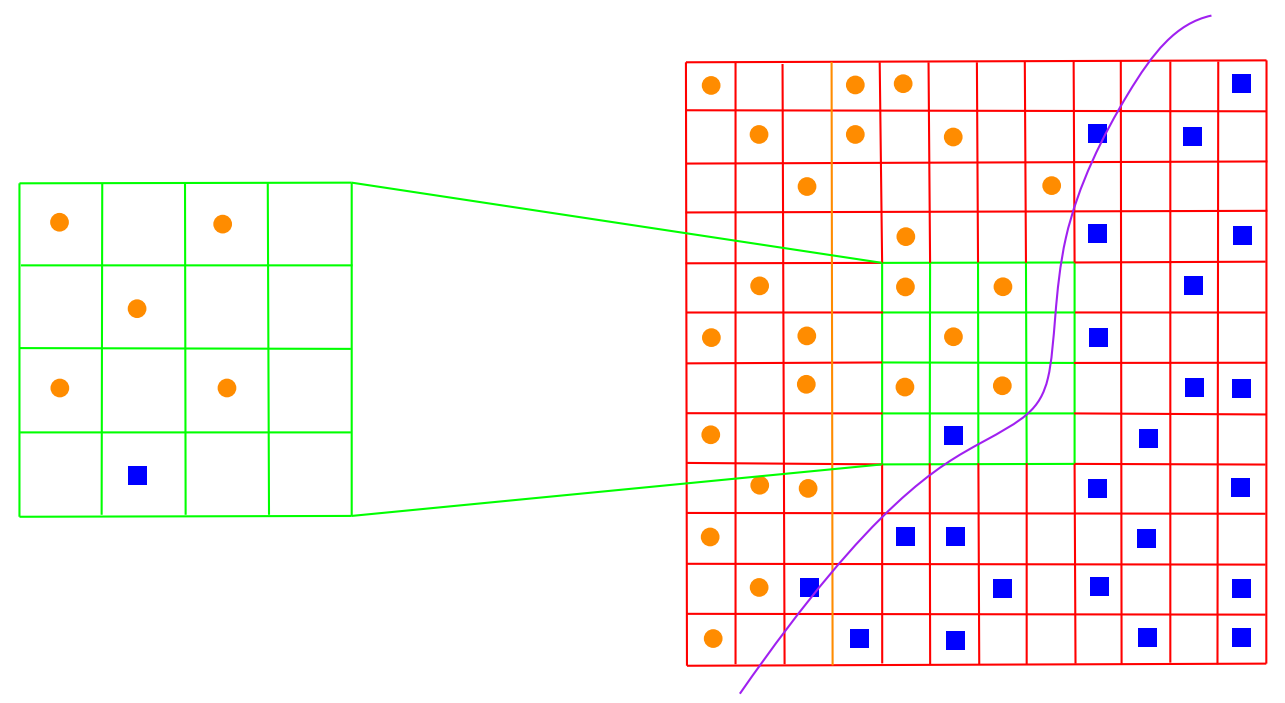

Figure 3.6: In a sub-pixel area, an edge may be difficult to localize correctly (left); the orange dots and the blue square(s) are rendered samples around an edge. But the orientation of the edge (purple line) becomes more clear when a sub-pixel patch is formed (right), even when the ratio of knownto-unknown samples remain constant.

where, $\Sigma_{i i}(X)$ is the $i^{t h}$ singular value of $X, \omega_{i}$ is the weight for the $i^{\text {th }}$ singular value, and $\epsilon$ is a small regularizer to avoid dividing by zero.

PAWNNM: For efficient optimization, we add a parameter adjustment (PA) strategy, reducing $\omega$ with a constant $c=0.9$ in each image recovery iteration, which can progressively increase recovery quality during the optimization loop.

$$
\omega_{i+1}=c \omega_{i}
$$

The PA strategy is based on the observation that recovered image quality keeps increasing within fixed iterations. Compared with a hard threshold, using a gradually reduced soft threshold helps to achieve a better result, as demonstrated by Mancera and Portilla [91]. Their idea is to solve a single gradient descent step while slowly reducing the threshold iter- 
Table 3.2: PSNR comparison for NNM, WNNM and PAWNNM under 20\% input pixels.

\begin{tabular}{lccc}
\hline Input 20\% pixels & NNM & WNNM & PAWNNM \\
\hline Bamboo & 30.74 & 31.01 & 32.29 \\
Bedroom & 37.85 & 39.35 & 39.96 \\
Bottles & 35.37 & 36.96 & 37.15 \\
\hline
\end{tabular}

atively. We compared our PAWNNM with NNM and WNNM in pixel recovery. Table 3.2 shows PSNR results of the three methods and our PAWNNM demonstrates better recovery.

Algorithm 1 shows the whole recovery process, based on our PAWNN$\mathrm{M}$ scheme. The inputs are the rendered data, $M$, and the corresponding sampling mask, $\Phi$, that the renderer has used to determine which samples to take. The first step in each iteration is to construct a low-rank matrix, $X$, using one of the schemes proposed in the previous section. We then apply our PAWNNM to recover the missing data. We iterate on this process to produce a good reconstructed result.

\subsection{Results}

\subsubsection{Experimental Setup}

Our method has been tested on various scenes including detailed scenes with high-frequency content for which it is challenging to recover missing information without introducing blurry artifacts. The test scenes include the Bamboo, Bedroom, and Bottles scenes in Figure 3.9, the San Miguel scene in Figure 3.8 and motion blur and soft shadow effect scenes in Figure 3.13. In order to create partially sampled rendering images as well 


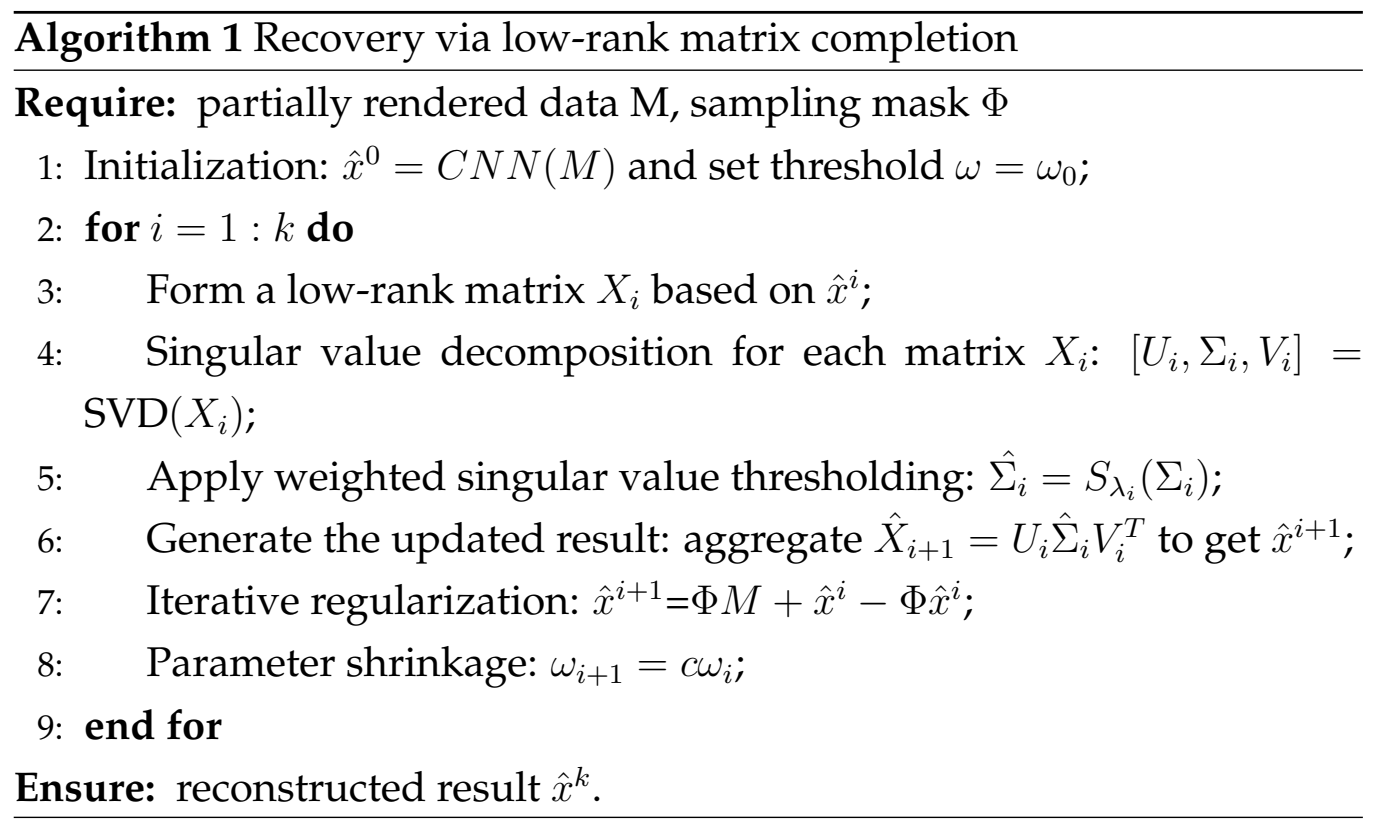

as the reference images, we use two open source renders, LuxRender and PBRT. The rendered static images are $1280 \times 960$ pixels, and animation sequences are $311 \times 500$ pixels. All of our experiments were performed on a workstation with a quad-core 3.0 GHz Intel Xeon CPU with 8G RAM. We set initial values by experiment based on accuracy and efficiency considerations. The initial values in Equation (3.14) are $\omega=80.5$, and $c=0.9$. The number of iterations in Algorithm 1 is set at $k=45$.

\subsubsection{Pixel Recovery}

Path Tracing rendering: A proportion of random pixels samples (20\%, $40 \%, 60 \%$ ) is selected and rendered using LuxRender and PBRT. Randomly selected pixels in each pixel patch $P_{p}(7 \times 7)$ are rendered and the positions are stored in a corresponding binary matrix. The patch size $(7 \times 7)$ has been selected experimentally while testing recovery quality of three different scenes under different patch sizes (Figure 3.7). The missing values of the patches are initialized by $\mathrm{CNN}$ and similar pixel patches within the image 


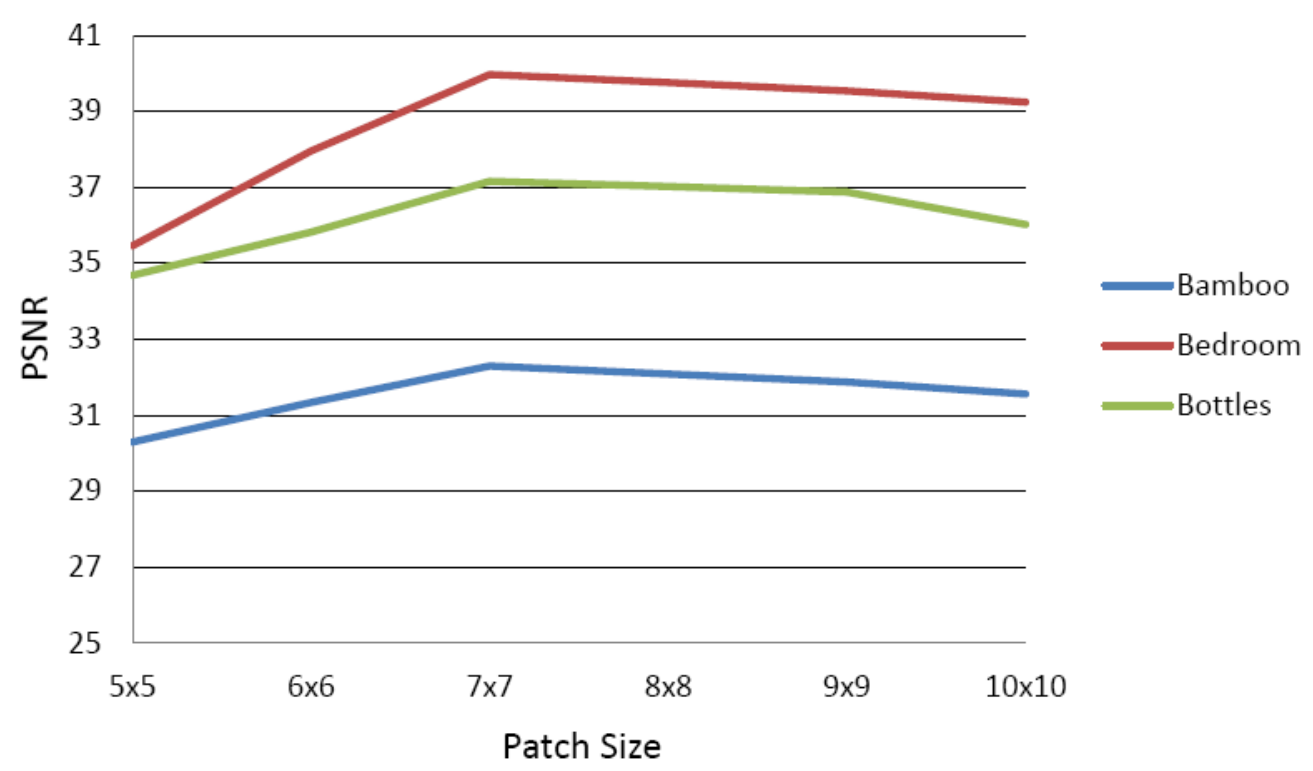

Figure 3.7: The corresponding PSNR of three test scenes with different patch size choices.

are grouped and create a low-rank matrix $M_{p}$ as explained in Section 3.2.1. Then, our reconstruction method estimates the values of missing pixels in $M_{p}$. After reconstructing values in a pixel patch, we process the next pixel patch while overlapping the patches by two pixels to avoid block artifacts. The size of the pixel patches are chosen by experiment to balance computation time and quality.

Figure 3.8 shows reconstructed images at different sampling rates $(10 \%$, $20 \%, 40 \%$ ). Even with low sampling rates, our method reconstructs missing samples efficiently and shows believable results required for previsualization.

The reconstruction results are compared with CS based reconstruction: CR [9] and CIR [23] in Figure 3.9 (we implemented CR ourselves and use the author's code for CIR). Our method with PAWNNM shows better results in terms of both visual quality and PSNR computed between reference and reconstructed images (see Table 3.3). CIR was compared with 

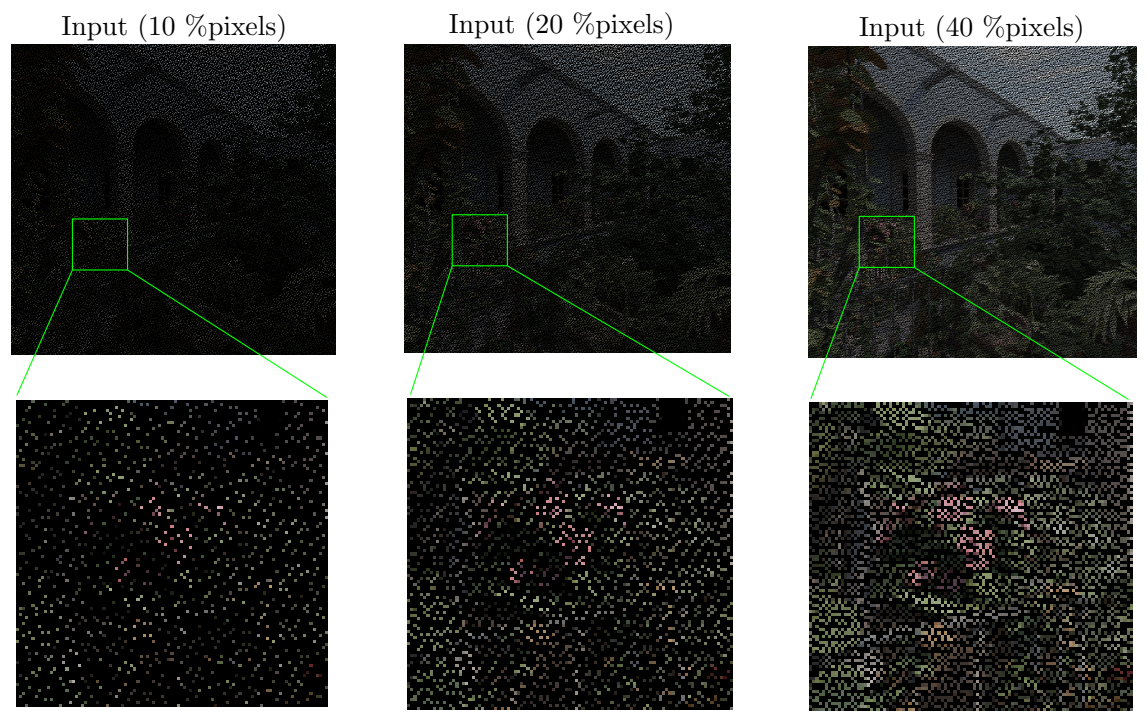

Recovery With $10 \%$ Pixels PSNR:22.58

Recovery With 20\% Pixels PSNR:28.93

Recovery With $40 \%$ Pixels PSNR:32.65
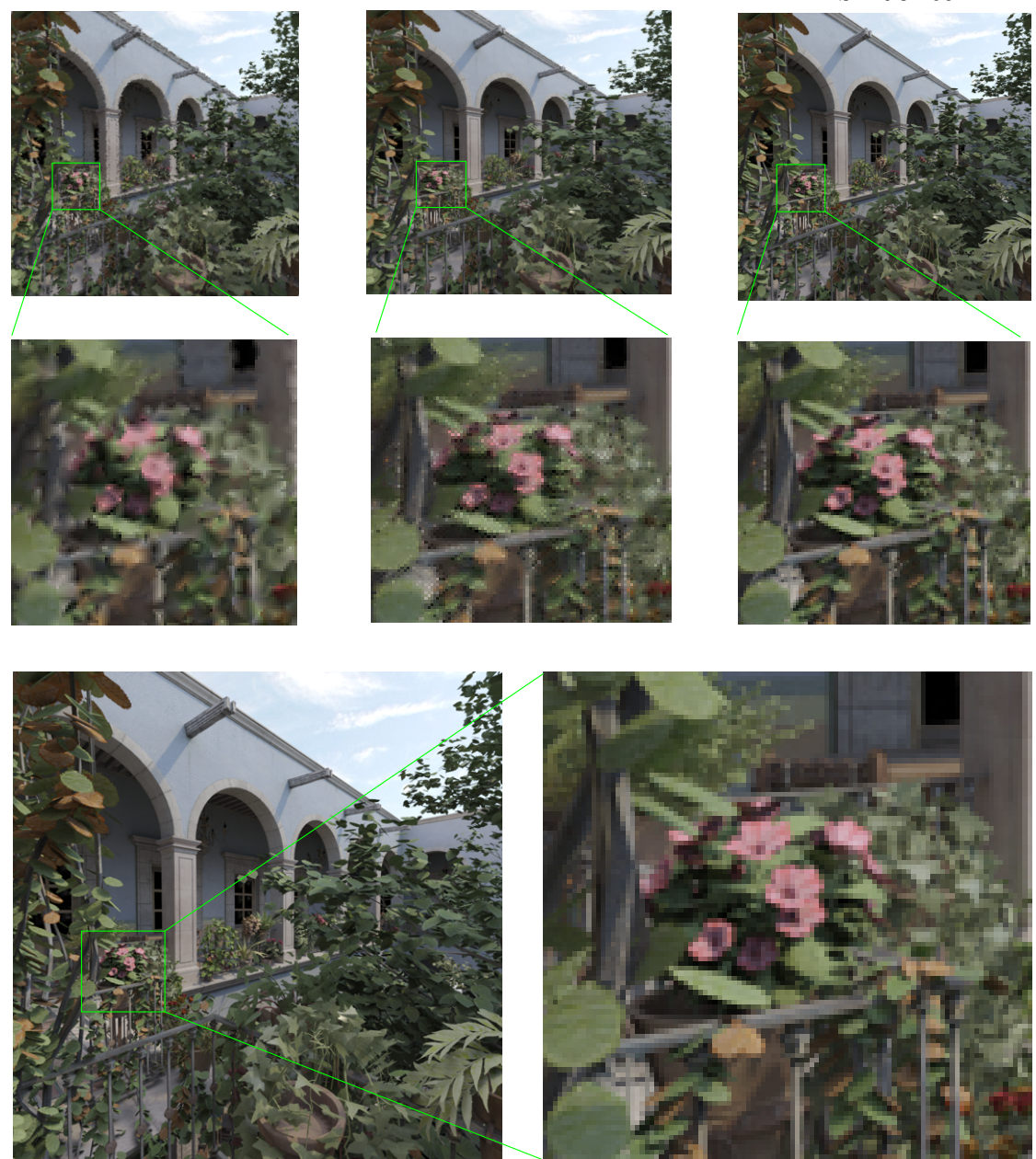

Reference

Figure 3.8: Examples of missing pixel recovery $(10 \%, 20 \%, 40 \%$ pixel samples). 


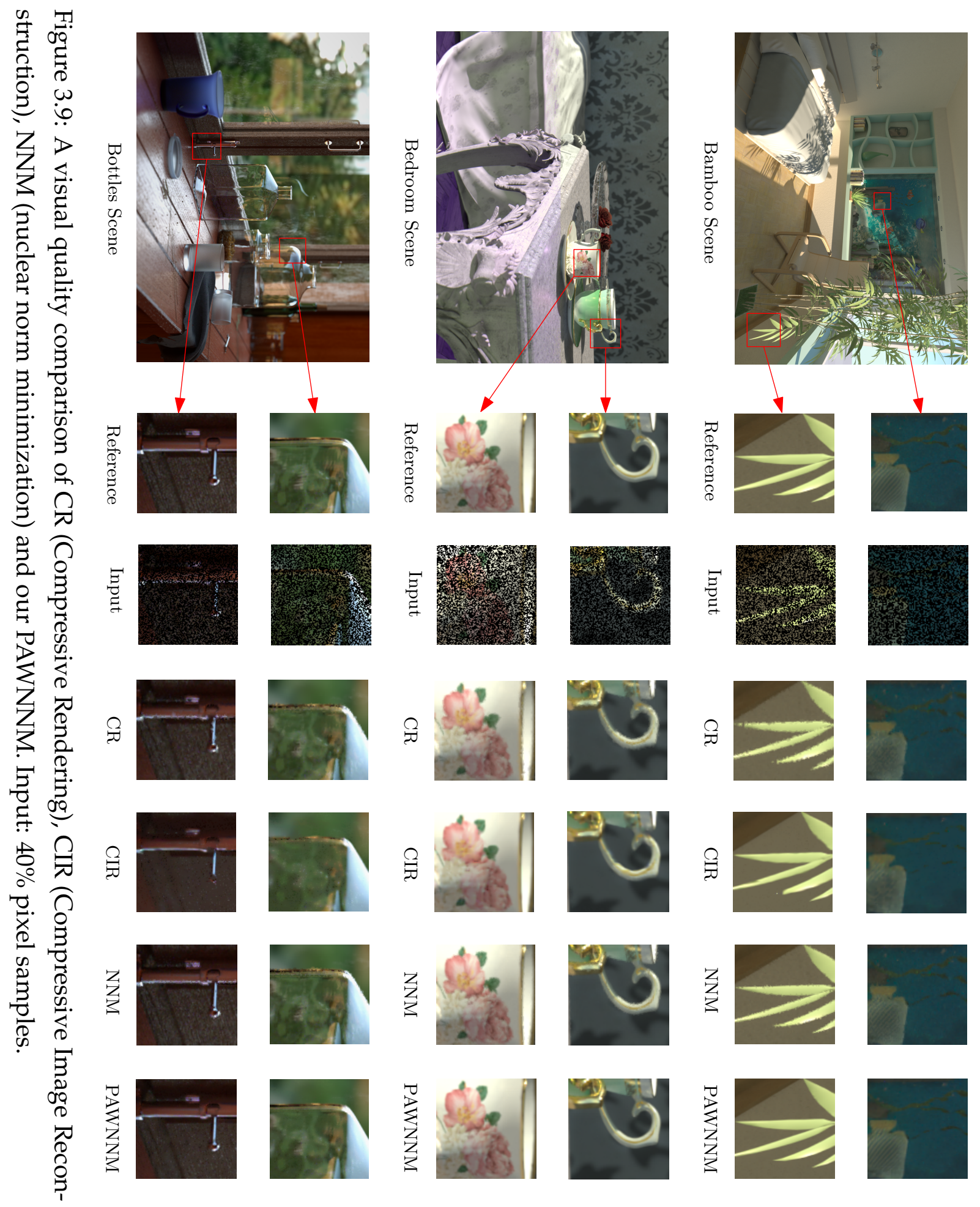


Table 3.3: PSNR/SSIM results of three test scenes under different sampling rates.

\begin{tabular}{|c|c|c|c|c|c|}
\hline Scene & Sampling Rate & CR & CIR & NNM & PAWNNM \\
\hline \multirow{3}{*}{ Bamboo } & $60 \%$ & $31.54 / 0.928$ & $38.33 / 0.965$ & $37.86 / 0.972$ & $40.62 / 0.980$ \\
\hline & $40 \%$ & $28.01 / 0.904$ & $34.56 / 0.953$ & $34.11 / 0.939$ & $36.65 / 0.962$ \\
\hline & $20 \%$ & $25.39 / 0.837$ & $30.81 / 0.917$ & $30.74 / 0.912$ & $32.29 / 0.931$ \\
\hline \multirow{3}{*}{ Bedroom } & $60 \%$ & $40.43 / 0.961$ & $47.15 / 0.984$ & 46.050 .982 & $48.68 / 0.990$ \\
\hline & $40 \%$ & $35.57 / 0.953$ & $43.54 / 0.974$ & $42.63 / 0.971$ & $45.32 / 0.985$ \\
\hline & $20 \%$ & $30.92 / 0.872$ & $38.37 / 0.956$ & $37.85 / 0.960$ & $39.96 / 0.967$ \\
\hline \multirow{3}{*}{ Bottles } & $60 \%$ & $34.25 / 0.906$ & $42.46 / 0.964$ & $41.69 / 0.957$ & $43.51 / 0.970$ \\
\hline & $40 \%$ & $32.19 / 0.865$ & $39.85 / 0.922$ & $39.02 / 0.920$ & $41.77 / 0.951$ \\
\hline & $20 \%$ & $29.56 / 0.801$ & $36.03 / 0.873$ & $35.37 / 0.911$ & $37.15 / 0.913$ \\
\hline
\end{tabular}

various filtering algorithms [7], [6], [19] in their paper [23] and showed better visual quality. Since our method outperforms CIR, by transitivity our methods have been compared with these three filtering methods.

Our recovery time depends on the size of the low-rank matrix and the number of similar pixel patches in an image. Using our test setups, the reconstruction time is around 17 minutes to recover missing pixel samples in $1280 \times 960$ images regardless the total number of samples. We have im-

Table 3.4: Relative reconstruction cost comparison under different sampling rates.

\begin{tabular}{lcccc}
\hline \multirow{2}{*}{ Scene } & Sampling Rate & CR & CIR & PAWNNM \\
\hline \multirow{3}{*}{ Bedroom } & $60 \%$ & $0.8 \%$ & $6 \%$ & $1.4 \%$ \\
\cline { 2 - 5 } & $40 \%$ & $0.8 \%$ & $5 \%$ & $1.4 \%$ \\
\cline { 2 - 5 } & $20 \%$ & $0.8 \%$ & $4 \%$ & $1.4 \%$ \\
\hline
\end{tabular}


Table 3.5: Given a fixed time budget (17 minutes), the PSNR of the recovery under CR, CIR and PAWNNM.

\begin{tabular}{lcccc}
\hline Scene & Sampling Rate & CR & CIR & PAWNNM \\
\hline \multirow{3}{*}{ Bedroom } & $60 \%$ & 40.43 & 47.15 & 48.68 \\
\cline { 2 - 5 } & $40 \%$ & 35.57 & 43.54 & 45.32 \\
\cline { 2 - 5 } & $20 \%$ & 30.92 & 38.37 & 39.96 \\
\hline
\end{tabular}

plemented CR on Linux and used the authors' code for CIR working on Windows. Due to different implementations, run time evaluation is not a fair comparison. Therefore, we used an relative reconstruction cost (reconstruction time/partial rendering time) for evaluation in Table 3.5. The efficiency of our PAWNNM is between CR and CIR, but the PAWNNM can achieve best reconstruction.

Inpainting: Although our main focus is to provide solutions for previsualisation of path tracing rendering, our method can be applied to inpainting. We have tested our method and compared the results with recent inpainting methods such as group-based sparse representation (GSR) [92] and non-local inpainting (NLI) [4].

We selected three standard test images (House, Boat and Hill) and compared the recovery results with $20 \%$ and $30 \%$ input random pixels. For GSR, we reference the author's implementation and NLI uses our implementation based on their paper. As shown in Table 3.6 and Figure 3.10 our method with PAWNNM outperforms the other two recent inpainting methods. Both the NLI and GSR fail to recovery the missing pixels without blurring image details, while our PAWNNM preserved more highfrequency information compared with the original images. More comparison results can be found in Appendix B. 


\subsection{RESULTS}

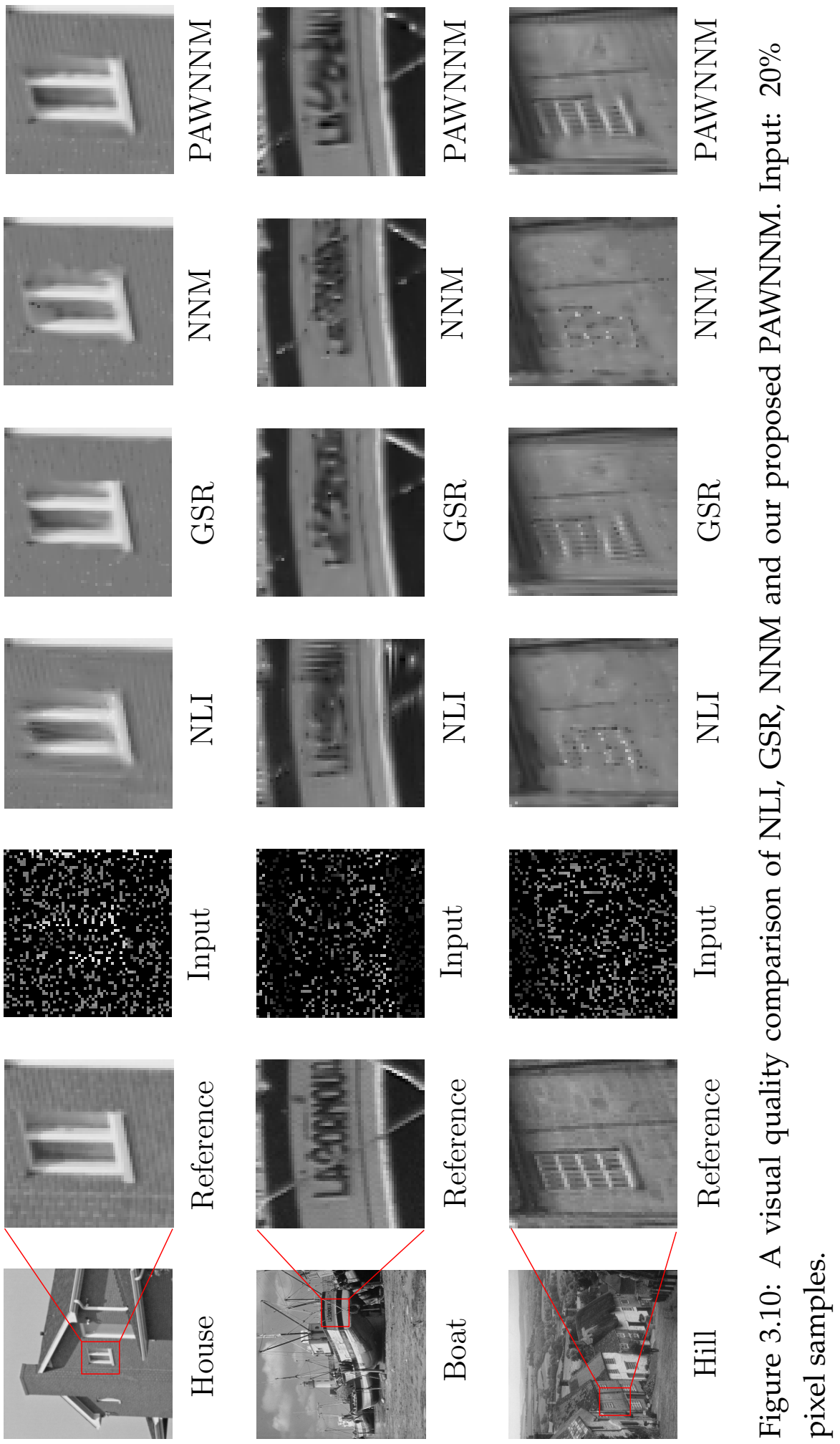



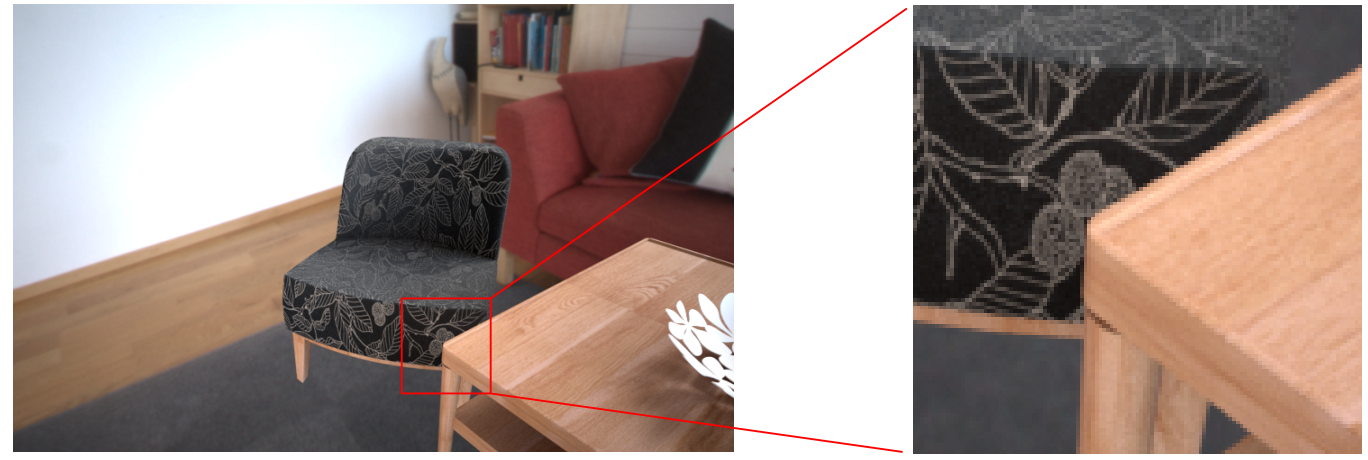

Reference

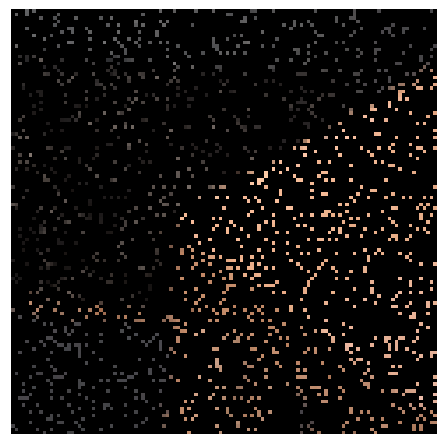

Input $10 \%$ pixels
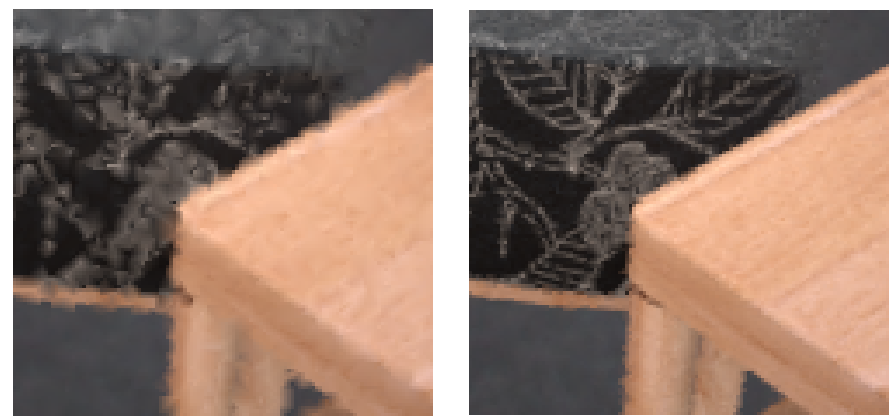

Recovery

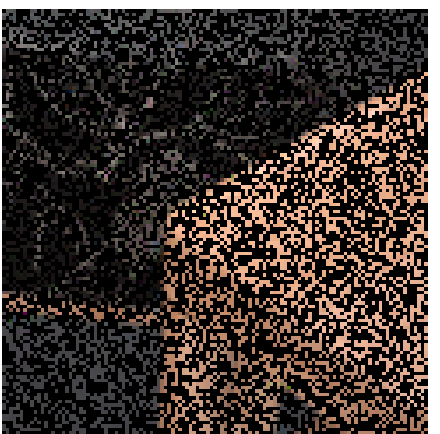

Input $40 \%$ pixels

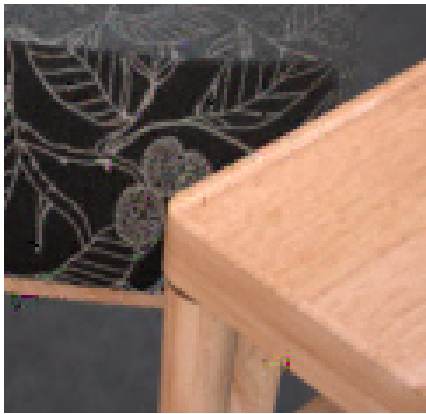

Figure 3.11: Example of multi-frame recovery (with PAWNNM) under different sampling rate. 
Table 3.6: PSNR/SSIM results of inpainting applications.

\begin{tabular}{lccccc}
\hline Image & Sampling Rate & NLI & GSR & NNM & PAWNNM \\
\hline \multirow{2}{*}{ House } & $30 \%$ & $33.83 / 0.893$ & $36.58 / 0.947$ & $36.06 / 0.941$ & $37.23 / 0.949$ \\
& $20 \%$ & $32.52 / 0.882$ & $34.36 / 0.903$ & $34.17 / 0.921$ & $35.47 / 0.927$ \\
\hline \multirow{2}{*}{ Boat } & $30 \%$ & $30.48 / 0.905$ & $31.36 / 0.911$ & $30.85 / 0.901$ & $32.74 / 0.928$ \\
& $20 \%$ & $28.23 / 0.876$ & $28.97 / 0.892$ & $28.39 / 0.884$ & $30.29 / 0.897$ \\
\hline \multirow{2}{*}{ Hill } & $30 \%$ & $31.31 / 0.889$ & $33.01 / 0.913$ & $32.02 / 0.906$ & $34.17 / 0.925$ \\
& $20 \%$ & $29.45 / 0.807$ & $31.44 / 0.891$ & $30.15 / 0.882$ & $32.56 / 0.908$ \\
\hline
\end{tabular}

\subsubsection{Multi-frame Recovery}

We have tested our method for recovering missing pixels in a video consisting of 147 frames, which is fully rendered in 24 hours. Portions of random pixel samples of each image are selected, rendered, and initialized as in Section 3.2.2. Pixel patches of size $7 \times 7$ are created and the similar patches in seven consecutive frames are selected and grouped. Ten similar patches are selected in each frame. Parameters in our test are selected by experiment to balance reconstruction time and visual quality. The selected similar patches construct a low-rank matrix $M_{m}$, and missing values of the matrix are estimated by our recovery method; boundary frames are overlapped and values are averaged.

The reconstructed result is shown in Figure 3.11. PSNR and SSIM measured between the reference and reconstructed images is shown in Table 3.7. Since we have reconstructed missing values in multiple frames concurrently while considering temporal coherence, potential temporal noise is minimized (multi-frame reconstruction shows lower standard deviation in Table 3.7). For frame-by-frame reconstruction, we used 70 similar patches per frame, but only 10 similar patches are used for multi-frame reconstruction. As shown in Table 3.7 our multi-frame reconstruction shows faster and better results than frame-by-frame reconstruction. The total re- 
Table 3.7: Result of multi-frame recovery (with PAWNNM): Incomplete video frames sampled at $10 \%, 20 \%$ and $40 \%$ are reconstructed independently (single-frame) and 7-frame concurrently (multi-frames). Time is measured in minutes.

\begin{tabular}{llllll}
\hline $\begin{array}{l}\text { Input } \\
\text { Samples }\end{array}$ & $\begin{array}{l}\text { Recovery } \\
\text { Method }\end{array}$ & $\begin{array}{l}\text { Average } \\
\text { PSNR/SSIM }\end{array}$ & $\begin{array}{l}\text { Standard } \\
\text { Deviation }\end{array}$ & $\begin{array}{l}\text { Recovery } \\
\text { Time }\end{array}$ & $\begin{array}{l}\text { Full Render } \\
\text { Time }\end{array}$ \\
\hline \multirow{2}{*}{$10 \%$} & single-frame & $27.42 / 0.867$ & 1.62 & 1029 & \\
\cline { 2 - 5 } & multi-frames & $29.38 / 0.872$ & 1.29 & 147 & \\
\cline { 2 - 5 } $20 \%$ & single-frame & $31.74 / 0.905$ & 1.25 & 1029 & \multirow{2}{*}{1440} \\
\cline { 2 - 5 } & multi frame & $34.23 / 0.931$ & 0.97 & 147 & \\
\cline { 2 - 5 } $40 \%$ & single-frame & $35.17 / 0.934$ & 1.08 & 1029 & \\
\cline { 2 - 5 } & multi frame & $39.41 / 0.956$ & 0.65 & 147 & \\
\hline
\end{tabular}

covery time is $10 \%$ of total rendering time.

\subsubsection{Sub-pixel Recovery}

For testing recovery of missing samples within a pixel, we assigned a uniform sub-pixel grid $G_{S}(i, j)$ having $16 \times 16$ elements to each pixel $(i, j)$. A fraction of the sub-pixel grid is randomly selected (10\% and $25 \%)$ and rendered using PBRT. Then, the grids of nearby pixels are grouped as a sub-pixel patch $P_{s} ; 3 \times 3$ nearby pixels are selected in our test to balance reconstruction time and quality. The low-rank matrix $M_{s}$ is constructed as in Section 3.2.3, and the missing sub-pixel samples are estimated by our matrix recovery step using PAWNNM. The reconstructed image of the San Miguel scene is shown in Figure 3.12. Our method efficiently recovers missing samples and shows promising results even in high-frequency regions. Our matrix completion contributes to provide efficient anti-aliasing in edge boundaries with fewer samples.

We compared our sub-pixel recovery results with three recent denoising filter techniques: ray histogram fusion (RHF) [2], learning based filter- 

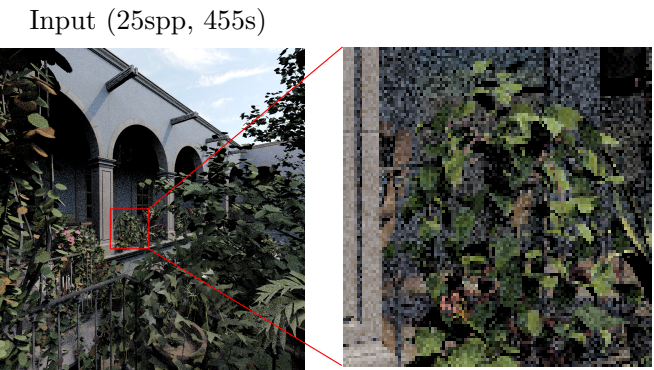

Input (64spp,1144s)

PAWNNM (25spp, 1195s)
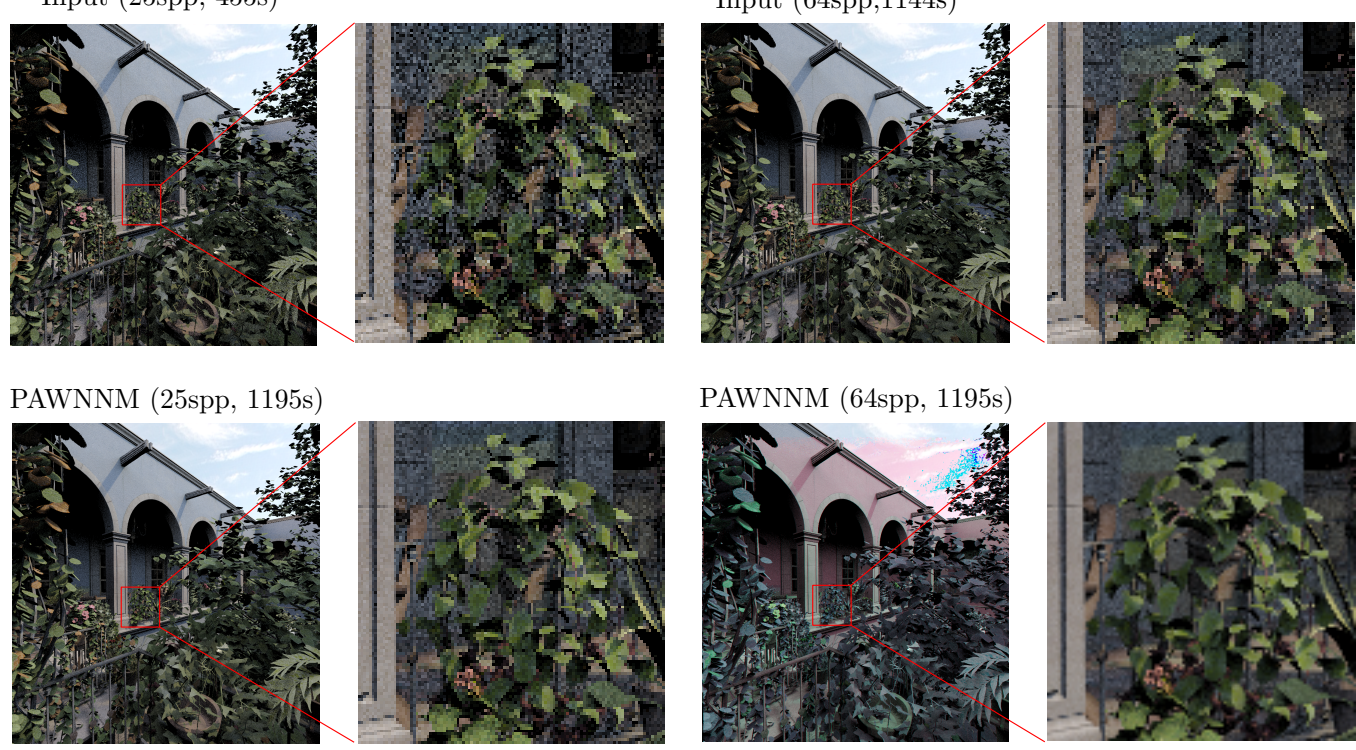

PAWNNM (64spp, 1195s)
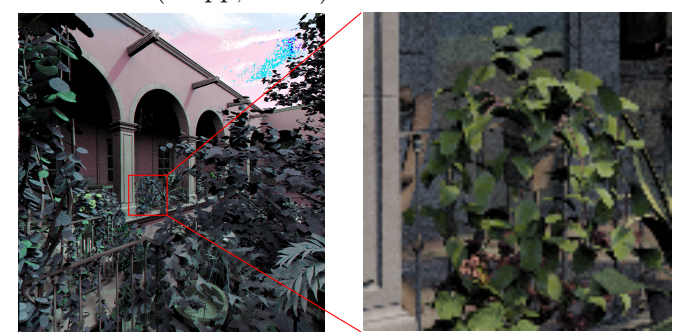

Reference (256spp,4570s)

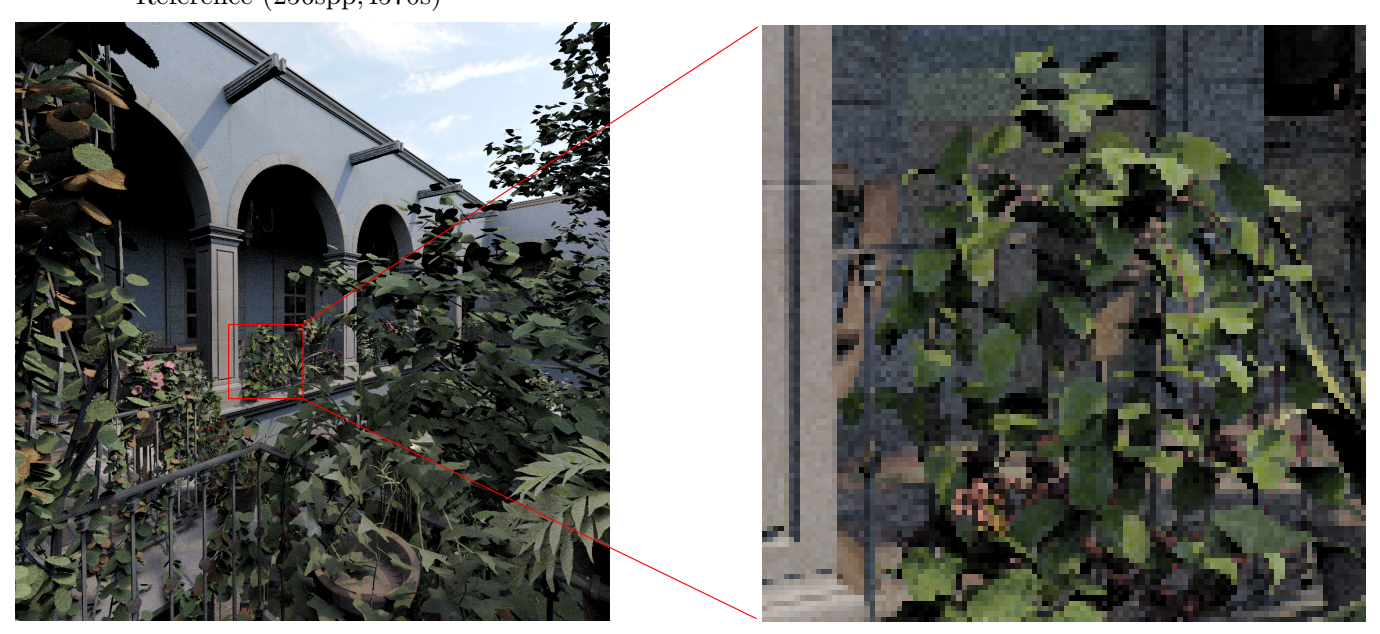

Figure 3.12: Examples of sub-pixel recovery under different sampling rates (10\% and $25 \%)$, From the top, input image rendered by 25 sub-pixels per pixel (spp) and $64 \mathrm{spp}$, our PAWNNM reconstructions, and the reference image rendered by $256 \mathrm{spp}$. The unit of rendering time (top and bottom), and reconstruction time is seconds. 
ing (LBF) [3], and Stein's unbiased risk estimator (SURE) [1] using incomplete input images with missing sub-pixel samples. As shown in Figure 3.13, our method achieves better results compared with RHF and SURE in all test scenes, and outperforms against LBF in scenes that have highfrequency detail, such as Teapot and Sponza. In our tests, LBF could not estimate appropriate filtering weights under a low sampling rate in these two scenes. We performed an equal time comparison with RHF and LBF (Figure 3.14): we fixed the total time including both rendering and recovery (or filtering) time. Within a fixed total time, RHF and LBF can have more input samples, since their filtering time is shorter than our recovery time (Table 3.8). Even with this advantage of more samples, RHF still shows worse results than our method. For the same total time, our method and LBF produce similar results. Since our current implementation is not optimal, our recovery time and the quality of the equal time comparison could be improved, as discussed in the following section.

Table 3.8: The time cost in equal time comparison test: RHF, LBF (rendering time/ filtering time) and ours PAWNNM (rendering time/ recovery time).

\begin{tabular}{llll}
\hline & RHF & LBF & PAWNNM \\
\hline Plants & $93 \mathrm{~s} / 90 \mathrm{~s}$ & $175 \mathrm{~s} / 15 \mathrm{~s}$ & $85 \mathrm{~s} / 100 \mathrm{~s}$ \\
\hline Sibenik & $168 \mathrm{~s} / 116 \mathrm{~s}$ & $260 \mathrm{~s} / 20 \mathrm{~s}$ & $107 \mathrm{~s} / 180 \mathrm{~s}$ \\
\hline
\end{tabular}

\subsection{Summary and Discussion}

In this chapter, we present an efficient reconstruction method for recovering missing values in an incomplete path tracing image using matrix completion. Our novel construction schemes efficiently create low-rank matrices for images having missing pixels, missing pixels in multiple frames, and missing sub-pixel samples within a pixel. Our CNN based pre-compl- 

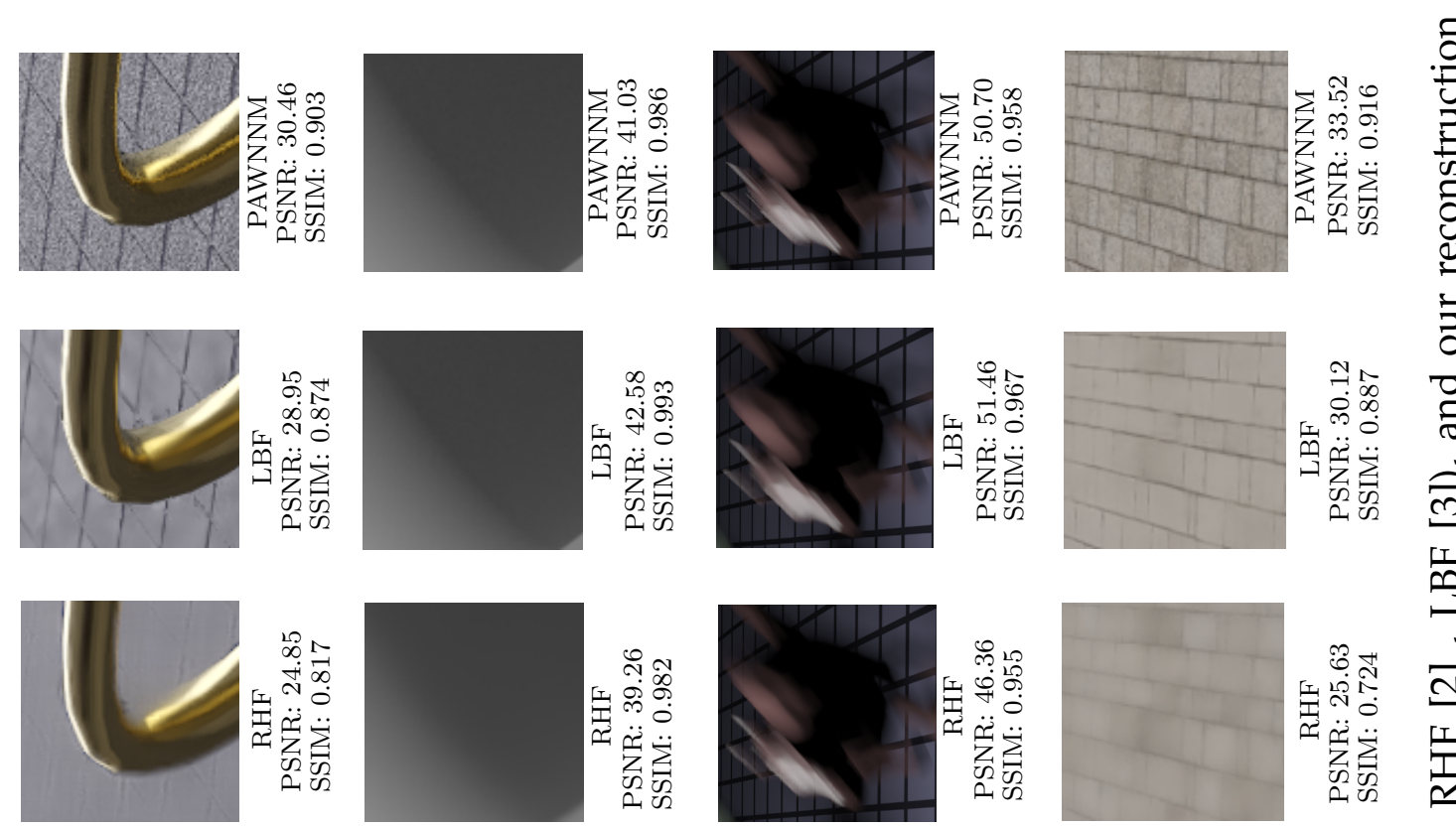

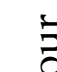
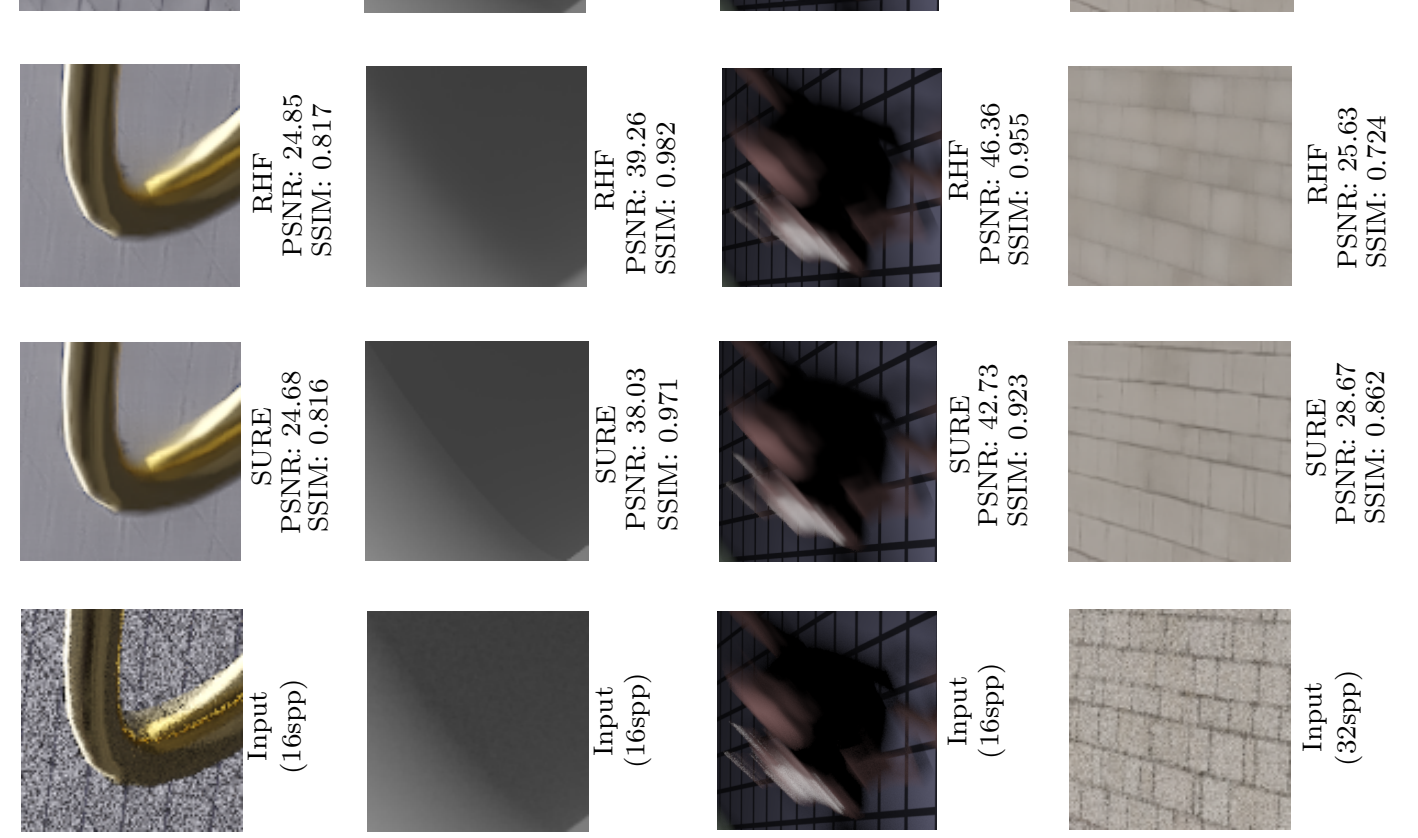

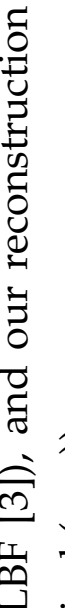
品 둴 至 눈 छ

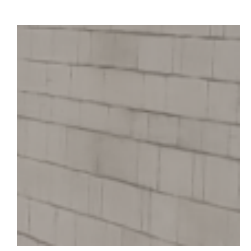

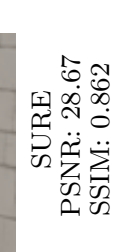
ए1 占

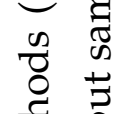
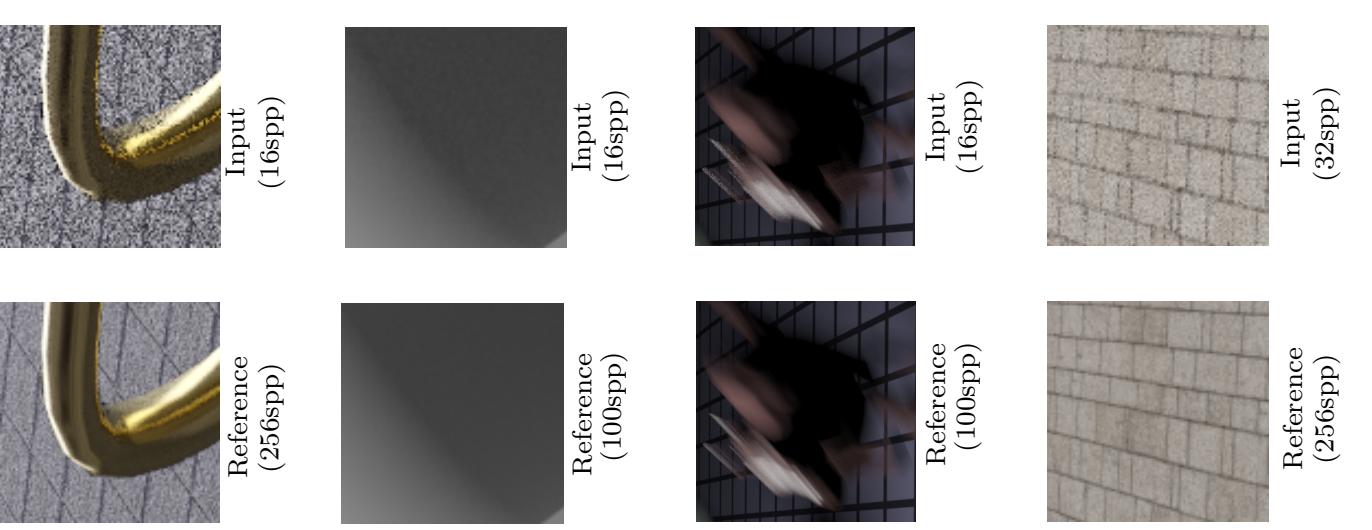

声 寻

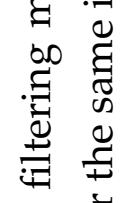
胥
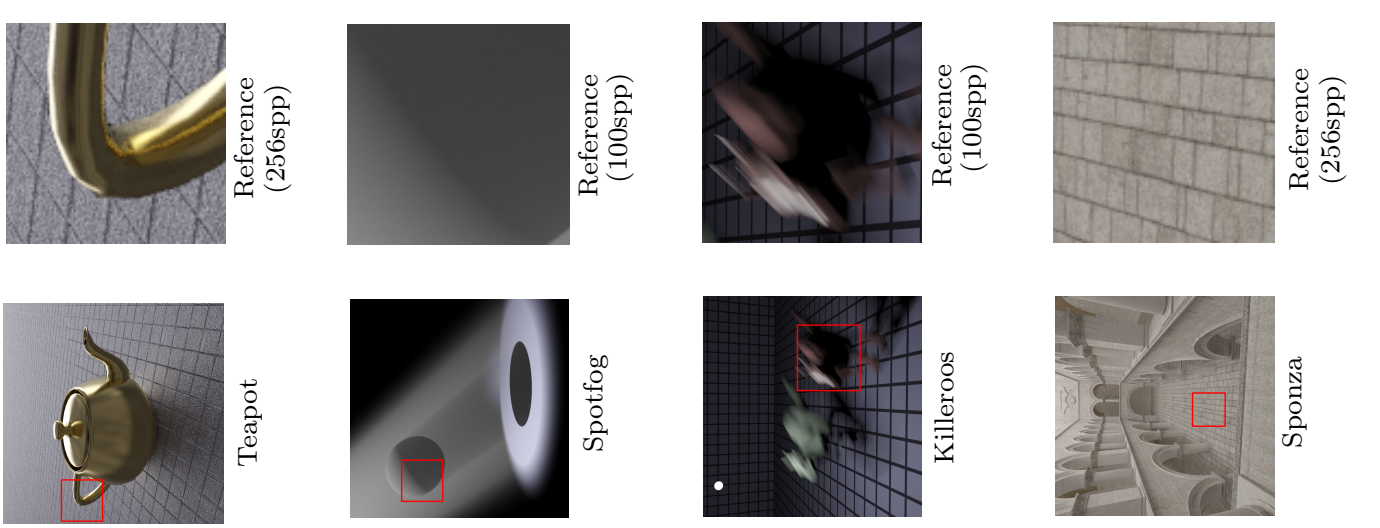

吾 

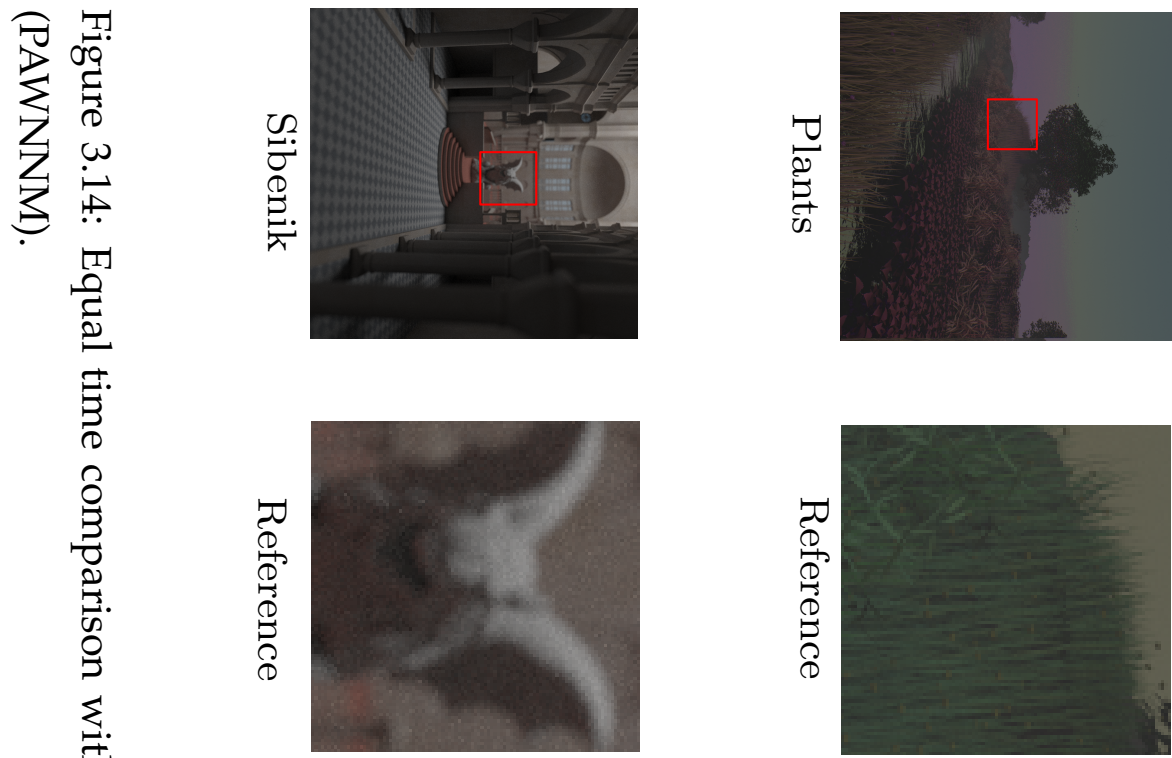

嶎

呬
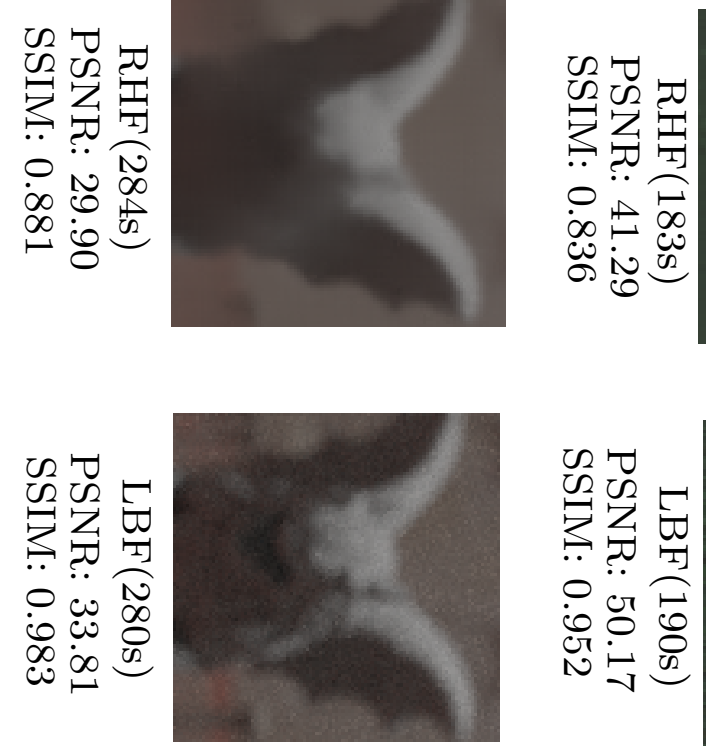

ơ
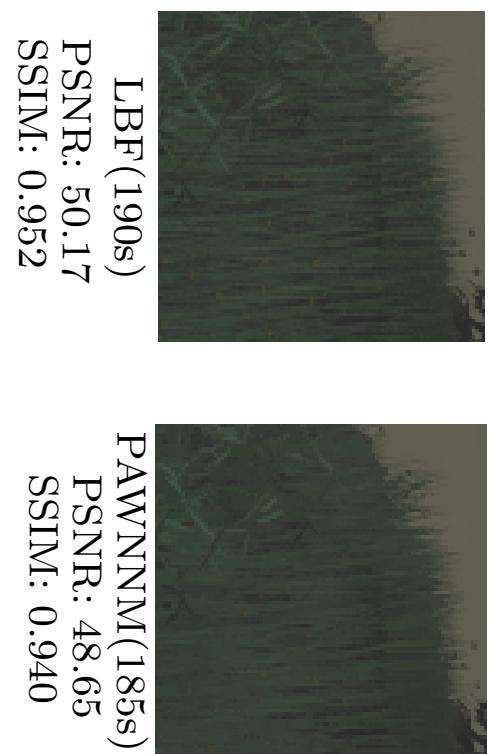
etion provides the fast initialization required to find similar patches in the incomplete image(s). Our multi-weighted NNM with progressive parameter adjustment strategy (PAWNNM) recovers missing values in low-rank matrices while recovering high-frequency details efficiently. Our method shows better reconstruction than recent compressed sensing based techniques and filtering methods at the same sampling rates.

Since artifacts in an intermediate image of path tracing can be caused by insufficient samples, we claim that matrix completion is an efficient choice as a screen space reconstruction for previsualisation. This is the first time matrix completion has been adapted for screen space reconstruction to recover missing values in sub-pixels and multiple frames for previsualization of path tracing. Our method provides an efficient image space solution to previsualize early stage intermediate results (e.g., $\leq 40 \%$ samples) with minimal blurry artifacts in high-frequency details, and to reduce temporal noise in a video. Furthermore, our PAWNNM shows promising results for inpainting providing better recovery compared with recent methods such as GSR [92] and NLI [4] in our experiments.

Although we show promising results, our method has some limitations. The performance of matrix completion relies on the size and number of low-rank matrices. Over several experiments, we determined appropriate patch sizes and an appropriate number of similar patches in an image or multi-frames, but careful analysis of scene information may provide better optimal choices. However, it is challenging to do this because, in general, detailed scene information cannot be known before a significant number of samples have been taken.

Our method is challenged when attempting to recover values under very low sampling rates $(<10 \%)$. Previous recovery methods, such as the compressive sensing (CS) based approaches, suffer from similar issues. Our method shows better and more reliable results than CS based approaches at very low sampling rates. 


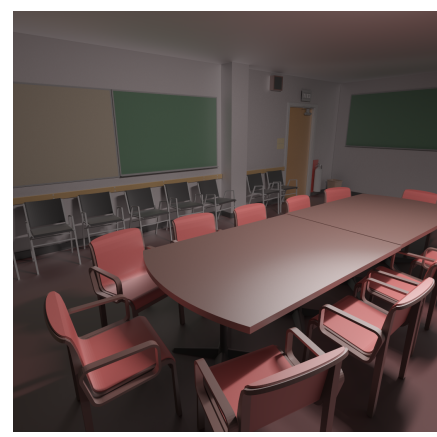

Conference

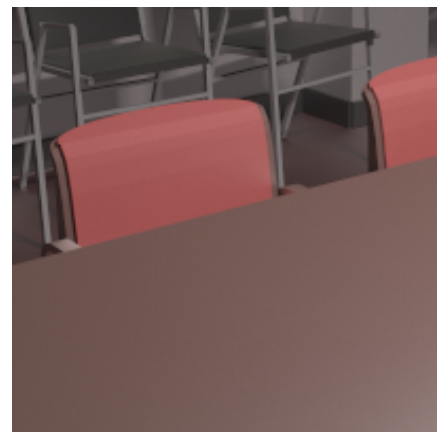

Pixel Recovery PSNR: 37.24 SSIM: 0.958

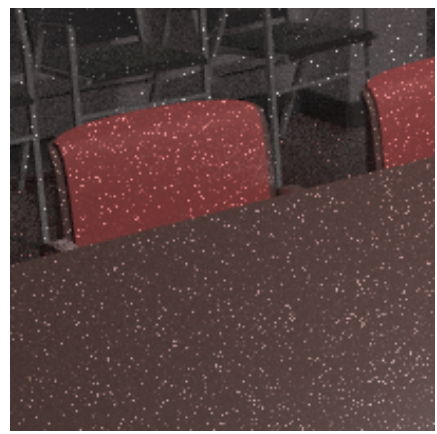

Sub-pixel Recovery PSNR: 32.69 SSIM: 0.873

Figure 3.15: A comparison between our pixel recovery and sub-pixel recovery under a same sampling rate $(20 \%)$.

We tested our method in three different recovery scenarios; pixel, multiframe, and sub-pixel. In pixel and multi-frame recovery, our reconstruction methods show reasonable relative reconstruction cost (i.e., reconstruction time $\leq 2 \%$ rendering time of input image). This performance is at least as good as CR or CIR and our recovery quality outperforms their results.

On the other hand, the current implementation of the sub-pixel recovery is not efficient compared with previous filtering methods in terms of the total time. Equal time comparisons (Figure 3.14) show that LBF performs better than our method. However, because the input images for the filtering methods will have more samples in the equal time case (Table 3.8), our method shows better recovery result under the same sampling rates (Figure 3.13). In terms of efficiency, there are two ways in which we could feasibly improve performance: (1) our current implementation is based on Matlab without careful code optimization, and (2) our reconstruction is fully independent across patches, and therefore each matrix recovery step could be computed in parallel on the GPU.

In our current implementation we have fixed the sub-pixel grid size at $16 \times 16$, since recovery time increases according to grid size, and we need to consider the relative reconstruction cost to balance the reconstruction 
and rendering time. Therefore, we have limitations to evaluate sufficient samples in complex scenes such as San Miguel (Figure 3.12).

We have tested the recovery results of pixel and sub-pixel samples under the same sampling rate (Figure 3.15). In general, computed values for pixel samples are more reliable, since each evaluated pixel has been averaged with relatively more samples, and therefore each pixel has a better chance to have less noise than sub-pixel samples. Since matrix completion relies on the computed values, the following recovery can provide better estimation for missing pixels.

In some challenging cases, filtering techniques may not output better quality image even with input having many more samples (e.g. 8000 samples per pixel). We performed equal time comparison between our pixel recovery and RHF to recover (or filter) an incomplete rendering of a complex scene (the Villa scene, Figure 3.16); in our experiment, LBF could not handle many samples due to its high memory requirement. The light source in this scene is located outside and therefore the scene requires a large number of samples for proper rendering. Under a fixed time budget, our pixel recovery method outperformed RHF. Therefore, for previsualisation of complex scene such as the Villa scene requiring many samples per pixel, our pixel recovery can be a better choice than filtering techniques. To this end, recall that our key target application, the movie post production pipeline, often requires rendering of very complex scenes with a large number of samples.

Since denoising filters can relieve the variance issue, a hybrid approach, combining filtering and reconstruction approaches, could be a future practical solution. 


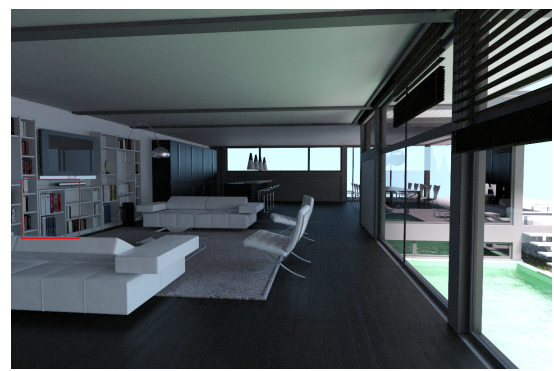

Villa

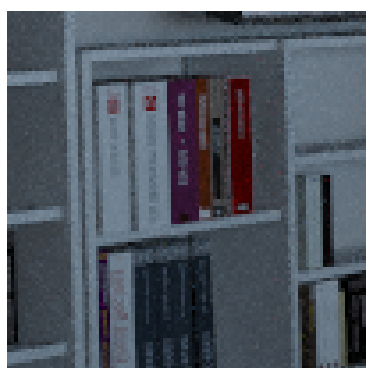

PAWNNM

PSNR: 36.57 SSIM: 0.945

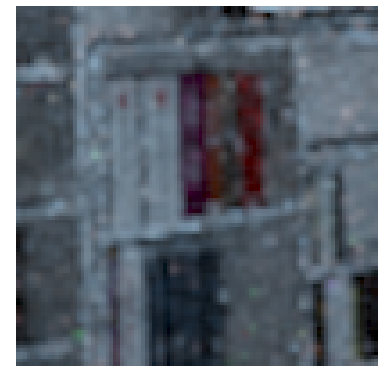

RHF

PSNR: 29.84 SSIM: 0.861

Figure 3.16: Under a fixed time budget (1.8 hours), our pixel recovery (in the middle) can generate better visual quality results than filter based method, RHF (on the right).

Overall, our method shows an efficient reconstruction of incomplete path tracing images within a reasonable time, which can provide efficient screen space previsualization acceptable for many applications including visual effects rendering for films. The main limitation is that the extra time is spent on grouping similar patches for pixel recovery, we will offer another solution in the next chapter. Additionally, the proposed multi-frames reconstruction can only explore limited coherence information within the selected local area across multi-frames. To utilize global information of animation data, we also provide a new approach to this issue in Chapter 4. 


\section{Chapter 4}

\section{Enhanced Completion for Path Tracing}

The method described in Chapter 3 delivered an accurate and efficient solution compared to other reference solutions. However, there are limitations in terms of pixel recovery and multi-frame recovery. First, the process of grouping similar patches to form a low-rank matrix in pixel recovery still requires extra time. Second, the coherence exploited in multiframe recovery is mainly within a local area across frames.

In this chapter, we present novel reconstruction methods that efficiently recover missing data in incompletely rendered images and multi-frames using enhanced matrix completion and tensor completion respectively. Both of the two techniques have been applied to problems in different areas, such as image processing [93] and machine learning [94], but has not been exploited in computer graphics area. Enhanced Matrix Completion $(\mathrm{EMaC})$ was proposed by Chen et al. [69]. The method is based on matrix completion and begins with converting the incomplete matrix into an enhanced form with Toeplitz or Hankel structure, then apply nuclear norm minimization (NNM) to compute the missing samples. Tensor completion explores the algebraic structure of high dimensional data. More recently, Kilmer et al. [18] proposed tensor singular value decomposition for ten- 
sor nuclear norm minimization, which demonstrates efficiency in recovery. Based on the two techniques, we apply different strategies to form a structure with low-rank property in pixel and multi-frame recovery.

For pixel recovery, we developed enhanced matrix reconstruction (EMR). Specifically, we form an enhanced pixel patch using a block Toeplitz structure [95]. Unlike our previous method in Chapter 3 that pre-completing the missing pixels and grouping similar patches together to obtain a low-rank matrix, the new enhanced pixel patch skips the computation of finding the similar patches. Therefore the whole recovery time could be reduced. We also adopt the multi-weighted nuclear norm minimization with progressive parameter adjustment strategy in Chapter 3, to efficiently recover high-frequency details.

For multi-frame recovery, we proposed to use tensor completion based reconstruction (TCR). The intuition is to treat the multi-frames as a tensor structure and use redundancy across nearby frames. We accordingly adopt tensor completion here rather than a frame by frame recovery scheme. Specifically, we utilize tensor singular value decomposition (t-SVD) for minimizing the tensor nuclear norm and apply our PAWNNM in the iterations. As a comparison with previous method in Chapter 3, the proposed method can speed up the recovery process and preserve better accurate details.

We begin this chapter by briefly revisiting the enhanced matrix completion and tensor completion (Section 4.1). We then introduce how to generate enhanced matrix structure and tensor structure for pixel and multiframe recovery respectively (Section 4.2). In section 4.3, the recovery algorithms for enhanced matrix completion and tensor completion are described. In addition, Section 4.4 discusses results on selected test data and conduct the comparison between the proposed methods and exiting methods. 


\subsection{Overview}

\subsubsection{Basic Notation}

This section introduces the essential notations used in this chapter. Specifically, vectors are represented as lowercase letters (like $x, y, z$ ); matrices are represented as uppercase letters (like $X, Y, Z$ ); and tensors are represented as calligraphy letters (like $\mathcal{X}, \mathcal{Y}, \mathcal{Z})$.

A three-way real tensor is a three-dimensional structure in $R^{n_{1} \times n_{2} \times \ldots n_{N}}$. A slice of a tensor is defined as a two-dimensional matrix by fixing two indices of the tensor. Specifically, $\mathcal{X}(i,:,:)$ stands for the $i$-th horizontal slice, where the first index is held constant for the tensor $\mathcal{X}$. Similarly, the $i$-th lateral and frontal slice are represented by $\mathcal{X}(:, i,:)$ and $\mathcal{X}(:,:, i)$ receptivity. Additionally, we use $\mathcal{X}^{i}$ as the notation of the the $i$-th frontal slice, $\mathcal{X}(:,:, i)$ of $\mathcal{X} . \hat{\mathcal{X}}=\operatorname{fft}(\mathcal{X},[], 3)$ stands for the Fourier transform along the third dimension of $\mathcal{X}$. While $\mathcal{X}=\operatorname{ifft}(\hat{\mathcal{X}},[], 3)$ stands for the inverse Fourier transform.

Especially, we denote the block diagonal matrix of a given tensor $\mathcal{X} \in$ $R^{n_{1} \times n_{2} \times n_{3}}$ as :

$$
B D(\mathcal{X})=\left[\begin{array}{lll}
\mathcal{X}^{(1)} & & \\
& \ddots & \\
& & \mathcal{X}^{\left(n_{3}\right)}
\end{array}\right]
$$

\subsubsection{Enhanced Matrix Completion}

The matrix completion problem has been demonstrated that exact completion can be handled with nuclear norm minimization, which is a convex relaxation of the rank minimization problem [17]:

$$
\begin{array}{ll}
\min _{X} & \|X\|_{*} \\
\text { s.t. } & X_{i j}=M_{i j}, \quad(i, j) \in \Omega
\end{array}
$$


where $M$ is the complete matrix, $X$ is an estimation to be solved by rank minimization constraint on observed samples, and $\Omega$ contains the positions of the observed samples. The nuclear norm $\|X\|_{*}$ is defined as $\|X\|_{*}=$ $\sum_{j=1}^{n} S_{j}(X)$ ( $n$ is the number of singular values of $X$ ), and $S_{j}(X)$ are singular values of matrix $X$.

In the work by Chen et al. [69], the authors proved that the effectiveness of the enhanced structure relies on the shift-invariance of harmonic structure, which promotes the application of Toeplitz structure to address compressed sensing problem in frequency domain. Different from their work, we explore the low rank property in image domain using the Toeplitz structure. Thus, given and enhanced matrix $X_{E}$ that was generated by block Toeplitz matrix as described above, the EMaC problem can be described as:

$$
\begin{array}{ll}
\min _{X} & \left\|X_{E}\right\|_{*} \\
\text { s.t. } & X_{i j}=M_{i j}, \quad(i, j) \in \Omega
\end{array}
$$

\subsection{Low Rank Matrix from an Image}

In order to solve matrix completion using NNM, we need to build a low rank matrix for $X$. We present a numerical method to build the low rank matrix using a block Toeplitz matrix (BTM) from a patch of an image having missing or damaged pixels to recover.

\subsubsection{Block Toeplitz Matrix (BTM)}

A Toeplitz matrix has been introduced by [96] in mathematics. The block Toeplitz structure is comprised of multiple Toeplitz matrices, providing an efficient numerical solution to create a low rank matrix. Chen et al. [69] adapt this structure to solve compressed sensing problems. To the best of our knowledge, this is the first time the BTM has been used for solving the 


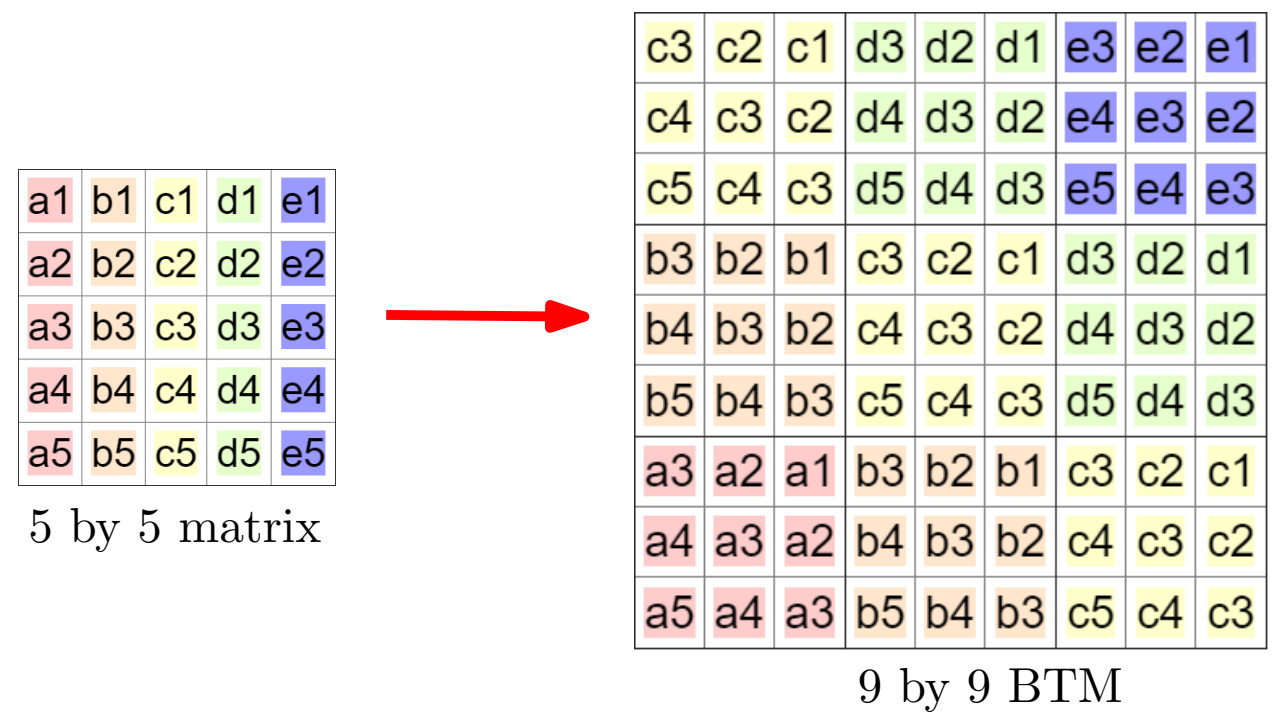

Figure 4.1: Given a 5 by 5 matrix, a corresponding BTM can be constructed from it (see Equation (4.4) and (4.5)).

matrix completion problem. A Toeplitz matrix is defined as:

$$
T=\left[\begin{array}{cccccc}
c_{0} & c_{-1} & c_{-2} & \cdots & \cdots & c_{1-n} \\
c_{1} & c_{0} & c_{-1} & \ddots & \ddots & \vdots \\
c_{2} & c_{1} & c_{0} & \ddots & \ddots & \vdots \\
\vdots & \ddots & \ddots & \ddots & \ddots & c_{-2} \\
\vdots & \ddots & \ddots & \ddots & c_{0} & c_{-1} \\
c_{n-1} & \cdots & \cdots & c_{2} & c_{1} & c_{0}
\end{array}\right]
$$

and the BTM, $X^{B}$, is defined using the blocks of the Toeplitz matrices:

$$
X^{B}=\left[\begin{array}{cccccc}
T_{0} & T_{-1} & T_{-2} & \cdots & \cdots & T_{1-n} \\
T_{1} & T_{0} & T_{-1} & \ddots & \ddots & \vdots \\
T_{2} & T_{1} & T_{0} & \ddots & \ddots & \vdots \\
\vdots & \ddots & \ddots & \ddots & \ddots & T_{-2} \\
\vdots & \ddots & \ddots & \ddots & T_{0} & T_{-1} \\
T_{n-1} & \cdots & \cdots & T_{2} & T_{1} & T_{0}
\end{array}\right]
$$




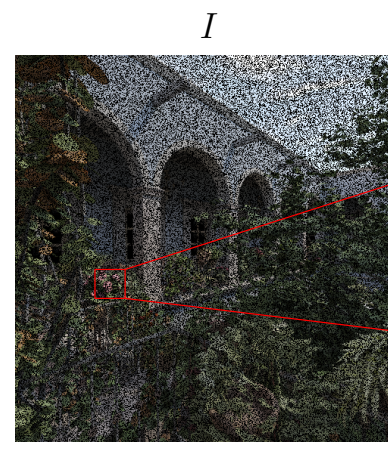

Input Image

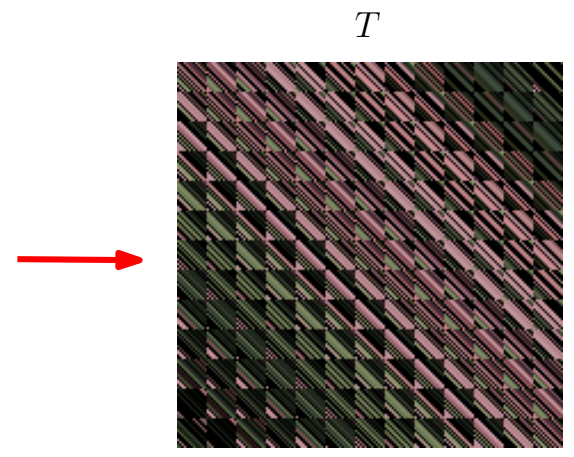

BT Matrix

Figure 4.2: Given a selected pixel patch $P_{i}$ from an incomplete path tracing image, a corresponding BTM can be constructed (see Equation (4.4) and $(4.5))$.

where $T_{i}$ of $X^{B}$ is a Toeplitz matrix. Figure 5.5 shows how to generate a BTM from a single 5 by 5 matrix.

\subsubsection{Create BTM from an Image}

Given an image patch $P_{i}$ with size $(2 n-1)$ by $(2 n-1)$ from the incomplete rendered image $I$, we form a block Toeplitz structure $X^{B}$ based on Equation (4.5). Specifically, each block $T_{i}$ is another Toeplitz matrix that is constructed from a column $C_{i}$ in patch $P$, the first row and column of $T_{i}$ are $\left(C_{i}^{0}, \ldots c_{i}^{1-n}\right)$ and $\left(C_{i}^{0}, \ldots c_{i}^{n-1}\right)$ respectively. The $2 n-1$ columns will generate $2 n-1$ Toeplitz matrices: $\left(T_{n-1}, \ldots T_{1-n}\right)$. Finally, the block Toeplitz matrix $X^{B}$ can be formed from the $2 n-1$ Toeplitz matrices, the first row and column of $X^{B}$ are $\left(T_{0}, \ldots T_{1-n}\right)$ and $\left(T_{0}, \ldots T_{n-1}\right)$ respectively. As shown in Figure 4.2, a selected patch from the partially rendered image can be transformed into block Toeplitz matrix. 


\subsection{Tensor Completion}

Given an incomplete tensor $\mathcal{X}$, the task of tensor completion is to reconstruct missing values using the known values, which can be described as the following convex optimization problem:

$$
\begin{array}{ll}
\min _{\mathcal{X}} & \|\mathcal{X}\|_{*} \\
\text { s.t. } & \mathcal{X}_{i j k}=\mathcal{M}_{i j k}, \quad(i, j, k) \in \Omega
\end{array}
$$

where $\mathcal{M}$ is the complete tensor data, and $\Omega$ contains the positions of the observed samples. $\|\mathcal{X}\|_{*}$ stands for the tensor nuclear norm of $\mathcal{X}$.

Previous methods, like the CP decomposition [97] and Tucker decomposition [98], which have been applied to solve the tensor completion problem, but both of the two techniques suffer from high computation or local minimal solution. More recently, Kilmer et al. [18] proposed tensorSVD to address the optimization problem (4.4), which is demonstrated as a more efficient and accurate solution for the tensor completion problem. Given a three-way tensor $\mathcal{X} \in R^{n_{1} \times n_{2} \times n_{3}}$, the t-SVD of $\mathcal{X}$ is defined as:

$$
\mathcal{X}=\mathcal{U} * \mathcal{S} * \mathcal{V}^{T}
$$

where $\mathcal{U}$ and $\mathcal{V}$ represent orthogonal tensors with size $n_{1} \times n_{1} \times n_{3}$ and $n_{2} \times n_{2} \times n_{3}$ respectively. While $\mathcal{S}$ stands for a diagonal tensor of size $n_{1} \times n_{2} \times n_{3}$, and ${ }^{*}$ denotes the tensor multiplication.

It has been proved that the tensor decomposition can be computed efficiently under Fourier domain by Kilmer et al. [18] and they use the Algorithm 2 to achieve the t-SVD product. Based on t-SVD, the tensor multi rank and tensor nuclear norm are defined as below:

Definition 1 (Tensor Multi-rank): The multi-rank of $\mathcal{X} \in R^{n_{1} \times n_{2} \times n_{3}}$ is a vector $r \in R^{n_{3} \times 1}$ with the $i^{t h}$ element equal to the rank of the $i^{t h}$ frontal slice of $\hat{\mathcal{X}}$.

Definition 2 (Tensor Nuclear Norm): The tensor nuclear norm of a tensor $\mathcal{X} \in R^{n_{1} \times n_{2} \times n_{3}}$ is defined as: $\|X\|_{*}=\|B D(\hat{\mathcal{X}})\|_{*}$. 


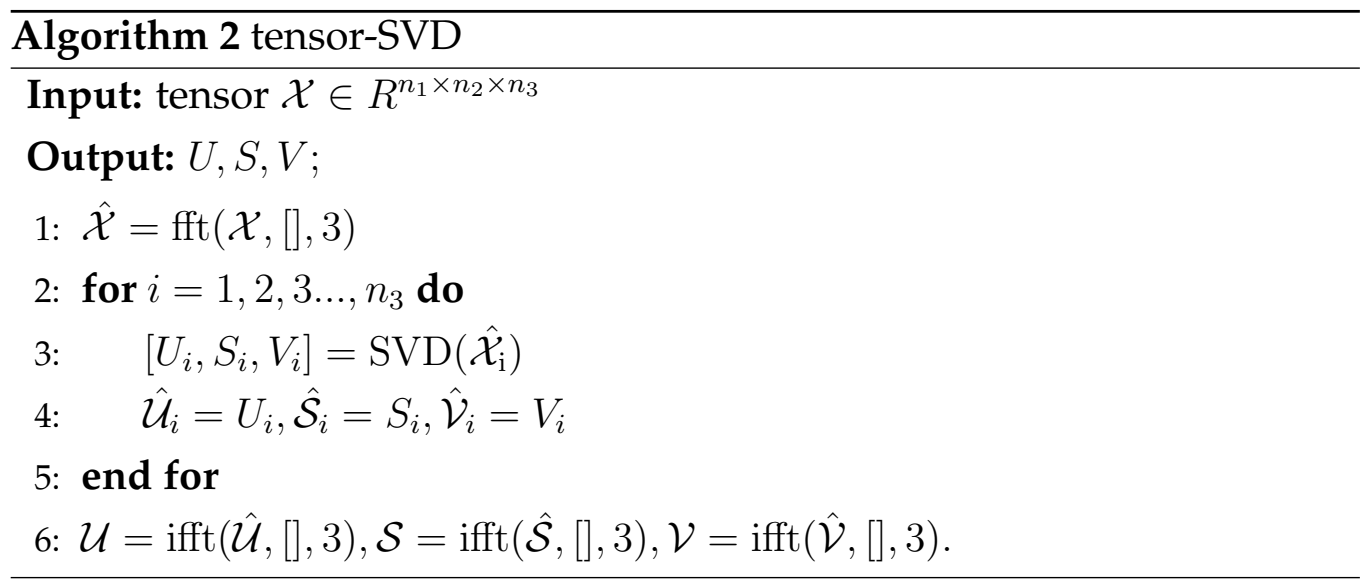

Thus, $\|B D(\hat{\mathcal{X}})\|_{*}$ can be computed through the multi-rank of $n_{3}$ subproblems: $\|B D(\hat{\mathcal{X}})\|_{*}^{*}=\sum_{i=1}^{n_{3}}\|\hat{\mathcal{X}}\|_{*}$

\subsubsection{Multi-frame Tensor}

Despite the single rendered image, multi-frame images in a video is another case that requires previewing by visual effects artists. Intuitively, a set of adjacent frames could be treated as a tensor product. By exploiting the redundant temporal information between frames, the missing samples could be efficiently recovered through the low-rank property of a tensor.

Given an incomplete rendered frame set $\left\{F_{1}, F_{2}, \ldots \ldots, F_{i}\right\}$, and each frame $F_{i}$ is partially rendered like our single image recovery case. Unlike the previous matrix completion method in Chapter 3, which find and group similar patches across adjacent frames to generate low-rank matrix, applying tensor completion can skip the grouping similar patches and directly exploit the low-rank property and coherence among continuous frames. As shown in Figure 4.3, a few adjacent partially rendered frames are grouped to form a tensor structure. 


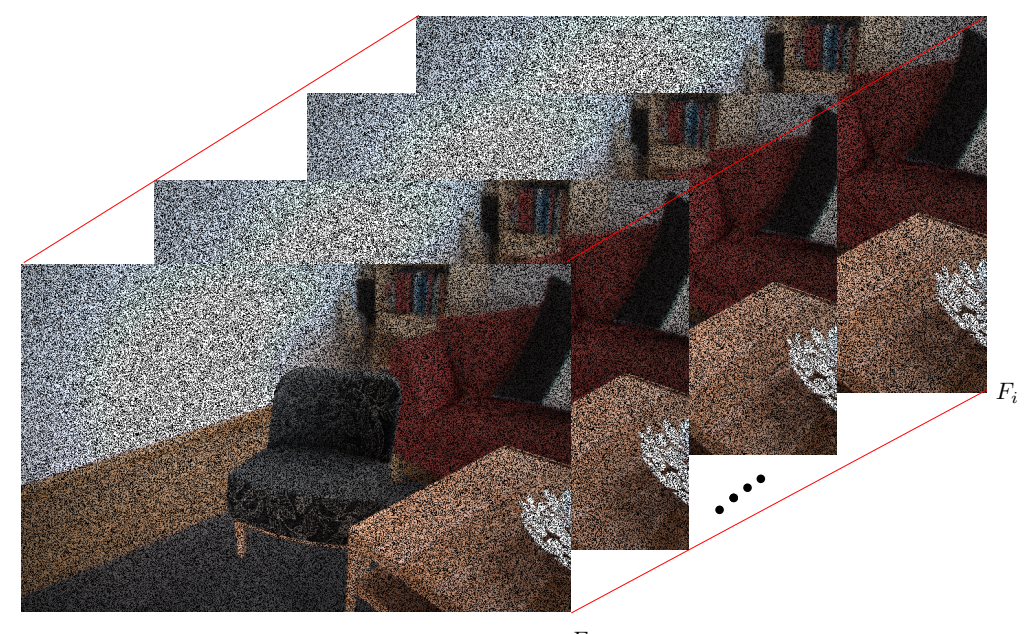

Figure 4.3: A tensor structure consists of a number of adjacent animation frames: $\left\{F_{1}, F_{2}, \ldots \ldots, F_{i}\right\}$.

\subsection{Enhanced Matrix and Tensor Recovery}

\subsubsection{Pixel Recovery via Enhanced Matrix Completion}

As we described at the beginning of this chapter, reconstruction for missing pixel samples involves two steps. First, generating an EM of a selected image patch of the rendering (Section 4.2). Then applying the enhanced matrix completion to recover the missing pixels, which will be addressed in this section.

Recall from Section 4.1, the enhanced matrix completion can be described as follow:

$$
\begin{array}{ll}
\min _{X} & \left\|X_{E}\right\|_{*} \\
\text { s.t. } & X_{E_{i j}}=M_{i j}, \quad(i, j) \in \Omega
\end{array}
$$

To efficiently solve the problem (4.8), we adopt the Alternating Direction Method of Multipliers (ADMM) [99]. It has been demonstrated that ADMM is high efficient for solving convex or non-convex problems in different application [99], the algorithm performs optimization in a low com- 
putational cost. Based on EMaC and ADMM, the augmented Lagrangian function of problem (4.8) could be defined as:

$$
L\left(X_{E}, Y, Z, \beta\right)=\left\|X_{E}\right\|_{*}-\left\langle Z, X_{E}-Y\right\rangle+\frac{\beta}{2}\left\|X_{E}-Y\right\|^{2}
$$

where $X_{E}$ is the estimate to be solved, $Y$ is an intermediate variable, $Z$ stands for the Lagrange multiplier, $\beta$ and $\langle$,$\rangle represent the penalty param-$ eter and the inner product respectively. The involved variables $X$ and $Y$ are computed separately in alternating order since the main concept of ADMM is to divide the minimization problem into two sub-problems that have closed-form solutions.

Specifically, the optimization problem (4.9) can be addressed as three sub-problems.

For computing $\boldsymbol{X}_{\boldsymbol{E}}$. We fix $Z_{k}$ and $Y_{k}$, and solve the minimization problem $L\left(X_{E}, Y_{k}, Z_{k}, \beta\right)$ as follows:

$$
\begin{aligned}
& X_{E_{k+1}}=\underset{X_{E}}{\arg \min } L\left(X_{E}, Y_{k}, Z_{k}, \beta\right) \\
& =\underset{X_{E}}{\arg \min }\left\|X_{E}\right\|_{*}+\beta / 2\left\|X_{E}-\left(Y_{k}-1 / \beta_{k}\right)\right\|^{2}
\end{aligned}
$$

We can obtain the closed form solution of (4.10) based on the weighted nuclear norm minimization (WNNM) [90]:

$$
X_{E_{k+1}}=W_{\lambda}\left(Z_{k}-\frac{1}{\beta} Y_{k}\right)
$$

where $W_{\lambda}$ is the weighted singular value shrink operator which is defined as follows:

$$
\begin{aligned}
& W_{\lambda}(X)=U D_{\lambda}(S) V^{T} \\
& D_{\lambda}(S)=\operatorname{diag}\left(\max \left\{S_{i}(X)-\lambda_{i}, 0\right\}\right) \\
& \lambda_{i}=\frac{\omega_{i}}{S_{i}(X)+\varepsilon}
\end{aligned}
$$

where $U, S, V$ are the SVD product of $X, S_{i}$ is the $i^{t h}$ singular value of $X$, $\omega_{i}$ is the weight for $i^{t h}$ singular value, and $\epsilon$ is a small regularizer to avoid dividing by zero. 
We followed the parameter adjustment (PA) strategy in Chapter 3, to efficiently solving the optimization problem. The PA strategy reduces the singular values with a constant parameter $C$, which is described as:

$$
\omega_{i+1}=c \omega_{i}
$$

As discussed and tested in Chapter 3, the PA strategy is based on the observation that recovered image quality keeps increasing within fixed iterations. Compared with a hard threshold, using a gradually reduced soft threshold helps to achieve an accurate result, as demonstrated by Mancera and Portilla [91].

For computing $\boldsymbol{Y}$. We fix $X_{k}, Z_{k}$ to calculate $Y_{k+1}$ as follows:

$$
\begin{aligned}
& Y_{k+1}=\arg \min L\left(X_{k}, Y, Z_{k}, \beta\right) \\
& =\arg \min \left\|Y-\left(X_{k}-\frac{1}{\beta} Z_{k}\right)\right\|^{2}
\end{aligned}
$$

which has a closed form solution, and we fix the values at the observed entries and obtain

$$
Y^{k+1}=\left\{\begin{array}{cc}
M_{i j} & \text { if }(i, j) \in \Omega \\
X_{k}-\frac{1}{\beta} Z_{k} & \text { otherwise }
\end{array}\right.
$$

For computing $Z$. We fix $X_{k}+1$ and $Y_{k}+1, Z_{k+1}$ which can be obtained from:

$$
Z_{k+1}=Z_{k}+\beta\left(X_{k+1}-Y_{k+1}\right)
$$

The whole procedure of the recovery is summarized in Algorithm 3.

\subsubsection{Multi-frame Recovery via Tensor Completion}

For multi-frames recovery, we treat a set of incomplete rendered frames as a tensor product $\mathcal{X}$. Based on the tensor nuclear norm and tensor-svd that introduced in Section 4.3, we describe the multi-frame recovery problem as:

$$
\begin{array}{ll}
\min _{\mathcal{X}} & \|\mathcal{X}\|_{*} \\
\text { s.t. } & \mathcal{X}_{i j k}=\mathcal{M}_{i j k}, \quad(i, j, k) \in \Omega
\end{array}
$$




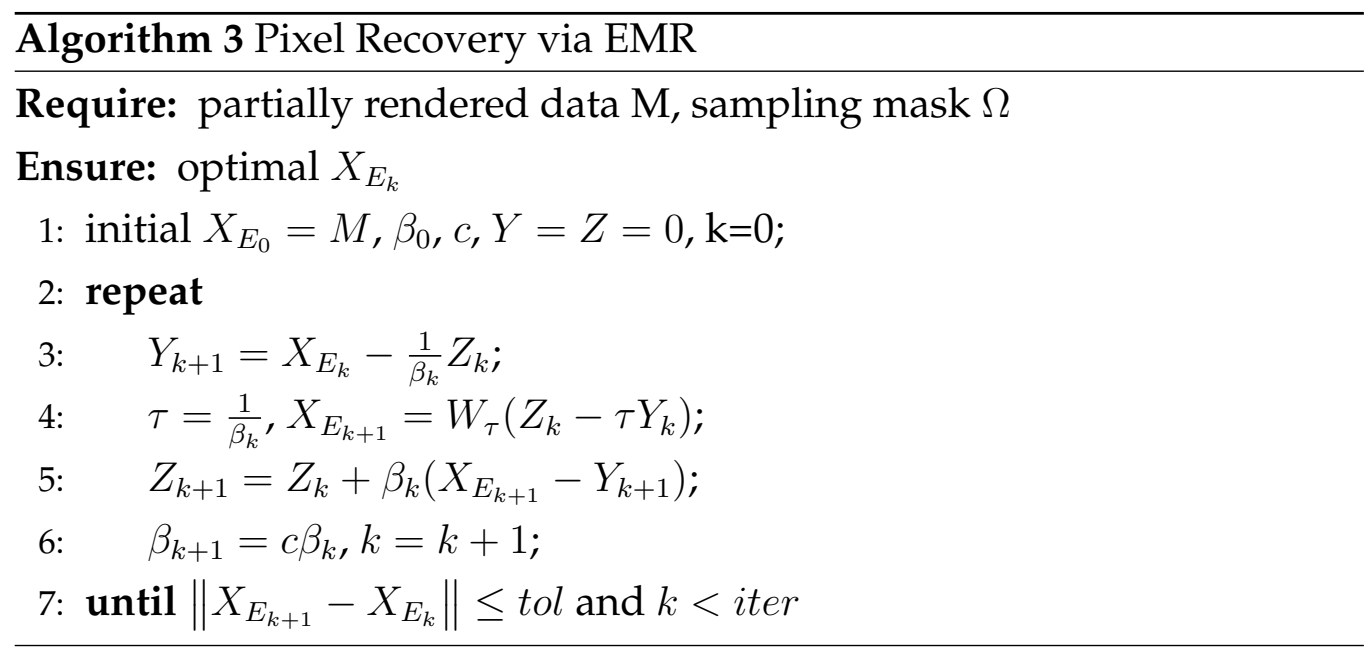

where $\mathcal{M}$ is complete tensor data, and $\Omega$ contains the positions of the observed samples. $\|\mathcal{X}\|_{*}$ stands for the tensor nuclear norm of $\mathcal{X}$.

Similar with the pixel recovery in previous section, we also adopt ADMM to solve Equation (4.17), and the objective function can be defined as:

$$
L(\mathcal{X}, \mathcal{Y}, \mathcal{Z}, \beta)=\|\mathcal{X}\|_{*}-\langle\mathcal{Z}, \mathcal{X}-\mathcal{Y}\rangle+\frac{\beta}{2}\|\mathcal{X}-\mathcal{Y}\|^{2}
$$

where $\mathcal{Z}_{k}$ and $\beta$ are the Lagrange multiplier and penalty parameter respectively, $\langle$,$\rangle stands for the standard inner product. \mathcal{Y}$ is an intermediate variable for computing $\mathcal{X}$.

The main difference between (4.18) and (4.8) is that the matrix term $X_{E}, Y, Z$ are replaced by tensor $\mathcal{X}, \mathcal{Y}, \mathcal{Z}$ accordingly. Thus, problem (4.18) can be iteratively solved by the three steps introduced in Section 4.3.1.

For computing $\mathcal{Y}$ and $\mathcal{Z}$, we can directly adapt the solution from (4.14), which is described as follows:

$$
\begin{gathered}
\mathcal{Y}^{k+1}=\left\{\begin{array}{cc}
\mathcal{M}_{i j k} & \text { if }(i, j, k) \in \Omega \\
\mathcal{X}_{k}-\frac{1}{\beta} \mathcal{Z}_{k} & \text { otherwise }
\end{array}\right. \\
\mathcal{Z}_{k+1}=\mathcal{Z}_{k}+\beta\left(\mathcal{X}_{k+1}-\mathcal{Y}_{k+1}\right)
\end{gathered}
$$

For computing $\mathcal{X}$, the minimization problem with tensor product is 
described as follows:

$$
\mathcal{X}_{k+1}=\underset{\mathcal{X}}{\arg \min }\|\mathcal{X}\|_{*}+\beta / 2\left\|\mathcal{X}-\left(\mathcal{Y}_{k}-1 / \beta \mathcal{Z}_{k}\right)\right\|^{2}
$$

Unlike like the pixel recovery case, here we utilize tensor-SVD with PAWNNM to solve $\mathcal{X}$.

$$
\begin{aligned}
& \mathcal{X}_{k+1}=\operatorname{ifft}\left(\hat{\mathcal{X}}_{\mathrm{k}+1}\right) \\
& \hat{\mathcal{X}}_{k+1}=\hat{W}_{\lambda}\left(\hat{Z}_{k}-\frac{1}{\beta} \hat{Y}_{k}\right)
\end{aligned}
$$

where ifft () stand for the inverse Fourier transform, $\hat{W}_{\lambda}$ stands for the weighted singular value shrink operator in Fourier space as follows:

$$
\hat{W}_{\lambda}(X)=W_{\lambda}(\hat{X})
$$

here, the definition of the weighted singular value shrink operator $W_{\lambda}$ is same with (4.11). The whole optimization process for multi-frame recovery is described in Algorithm 4. The input data are the partially rendered animation sequences, $\mathcal{M}$, and the corresponding sampling mask that the renderer has used to determine which samples to take. We iterate on the process based on the description in this section to generate a satisfying reconstructed result. 


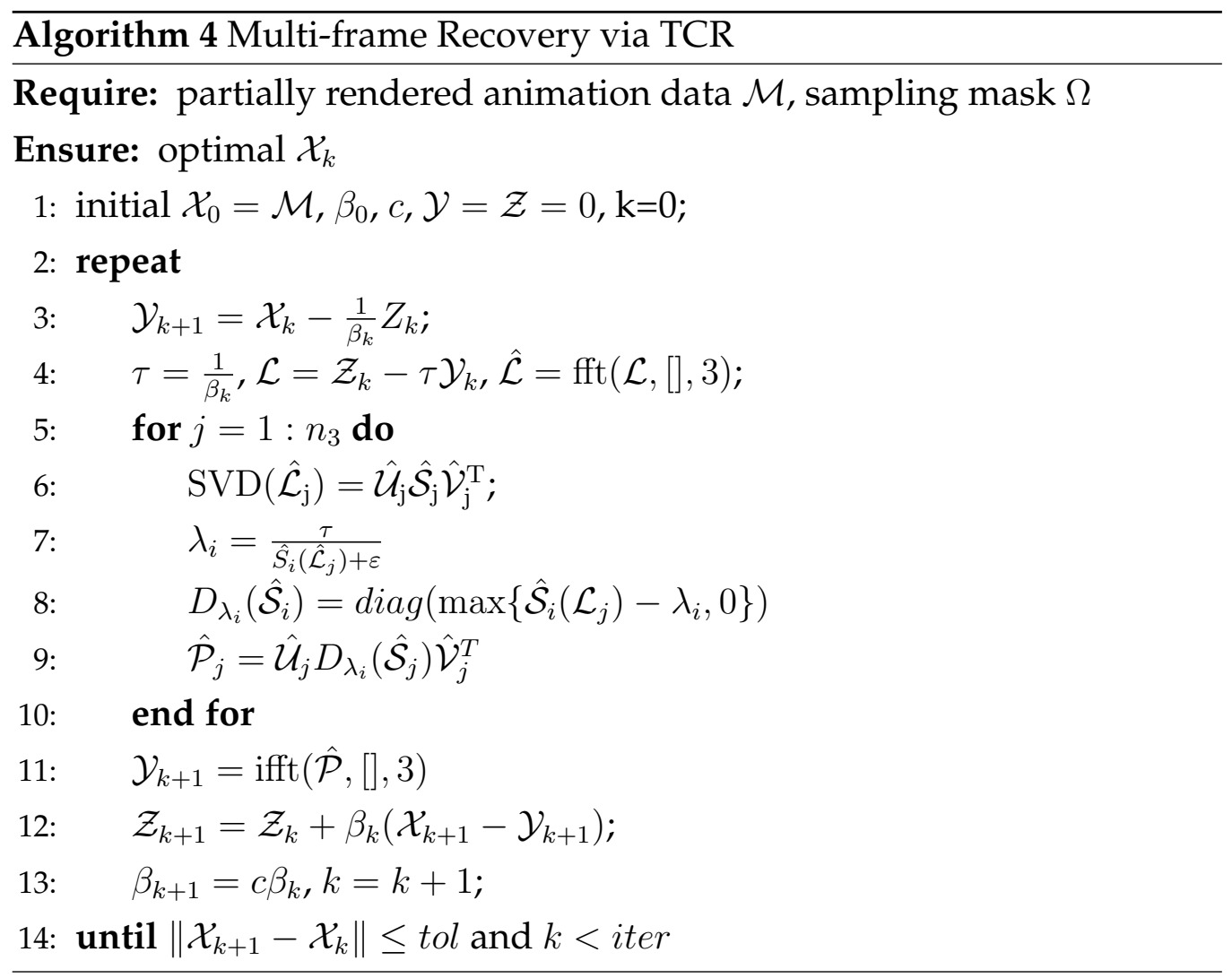

\subsection{Results}

We evaluated our method using various test scenes, including the Bedroom, Bamboo and Bottles scenes. Due to all these scenes contain highfrequency content, recovering missing samples without blurry artifacts would be challenging. Similar to the experiment setting in Chapter 3, the PBRT and LuxRender are applied to generate the partially rendered images or animated sequences. The tested images for pixel recovery are $1280 \times 960$ pixels, and animated sequences are $311 \times 500$ pixels. All experiments are conducted on a desktop with a quad-core 3.0 GHZ Intel Xeon CPU with 8G RAM. 


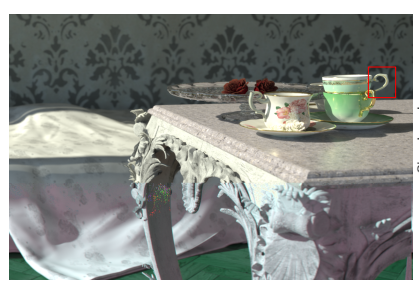

Bedroom

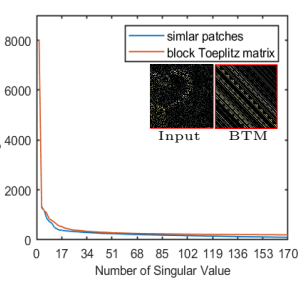

$20 \%$ Input pixels

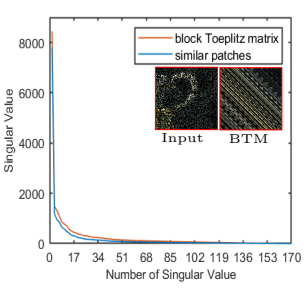

$40 \%$ Input pixels

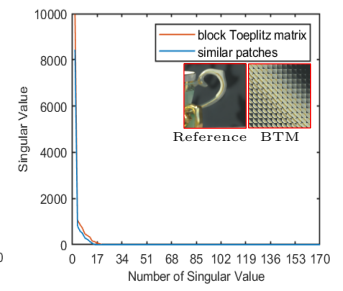

Reference

Figure 4.4: Given a selected patch, the block Toeplitz matrix is able to obtain a low rank property, similar to the patch grouping approaches; tested using a different percentage of randomly distributed input pixels (20\%, $40 \%$, and $100 \%$ as a reference).
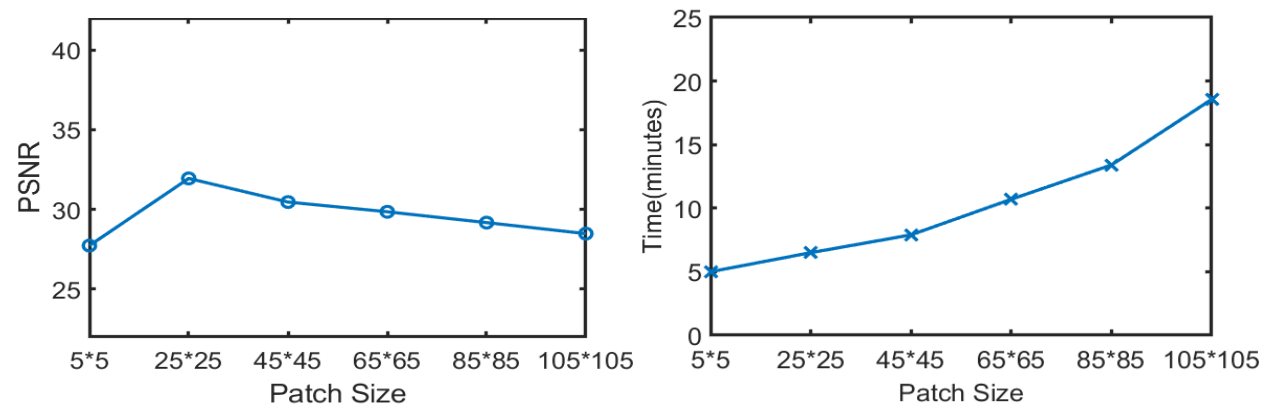

Figure 4.5: Recovery quality and time of an incomplete path tracing image (San Miguel scene with $1024 \times 1024$ resolution with $40 \%$ pixels) under different patch sizes using a BTM; we select $25 \times 25$ to balance quality and recovery time.

\subsubsection{Pixel Recovery}

We evaluate our method with missing pixels of an incomplete path tracing image and partially damaged photograph. In our tests, we use PBRT [100] to render images with missing pixels, and the quality is quantitatively measured using the PSNR and SSIM image similarity metrics. All experiments are conducted on a desktop with a quad-core 3.0 GHZ Intel Xeon CPU with 8G RAM. In our tests, we set the patch to $25 \times 25$, which we chose experimentally while testing recovery quality and time from eight different scenes under different patch sizes (Figure 4.5). The parameters 

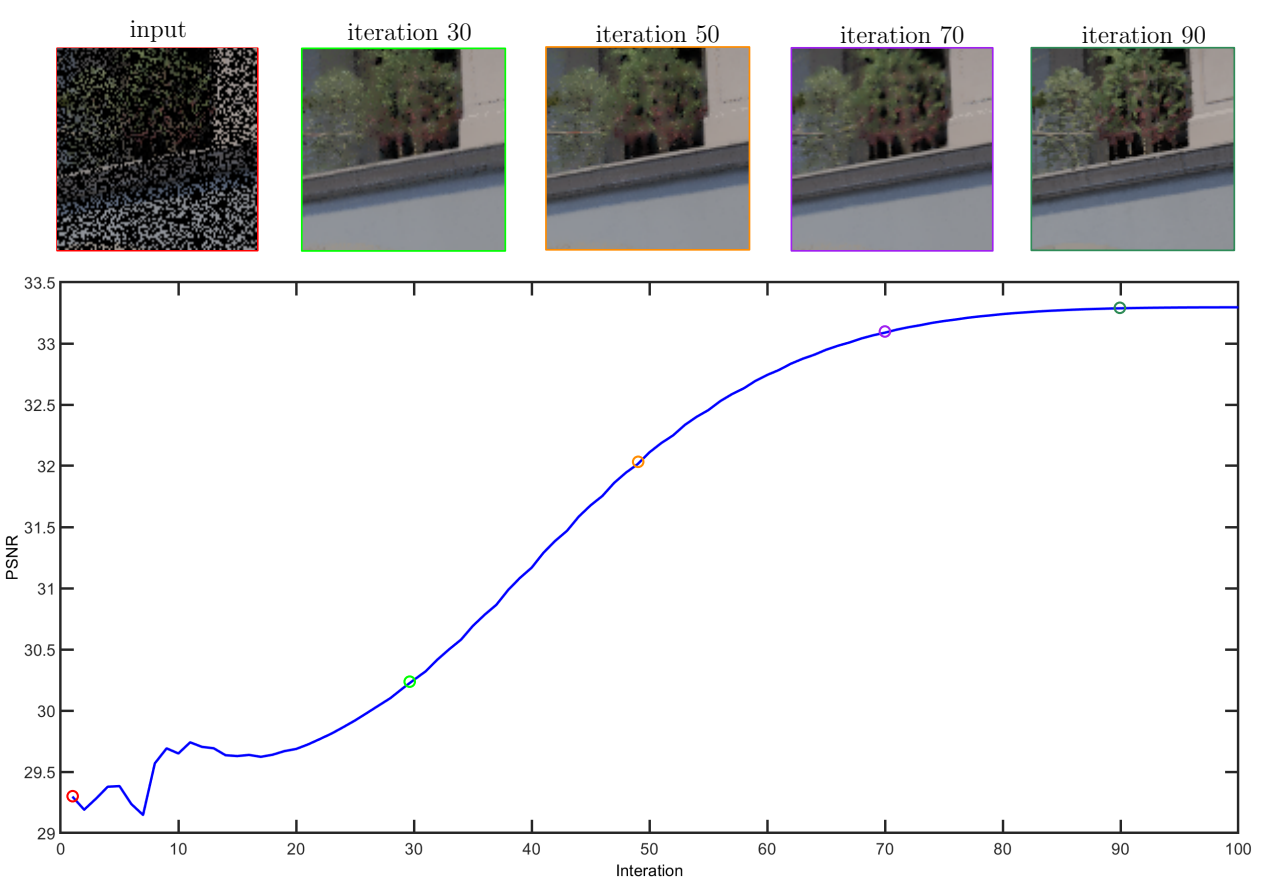

Figure 4.6: The PSNR of progressive recovery using iterative WNNM, going up to 100 iterations. This uses the Sanmiguel scene with $40 \%$ rendered pixels.
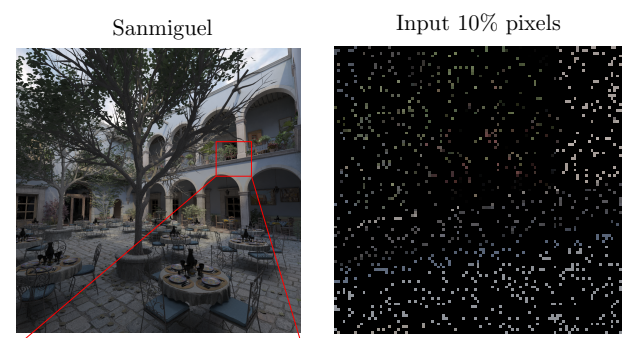

Input $20 \%$ pixels

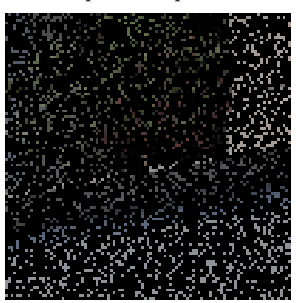

Input $40 \%$ pixels

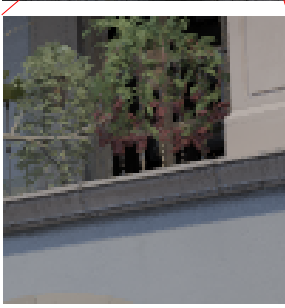

Reference

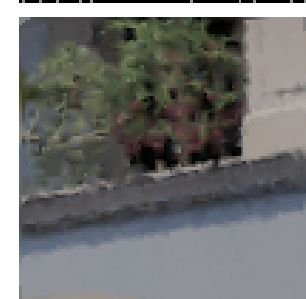

Recovery with $10 \%$ pixels

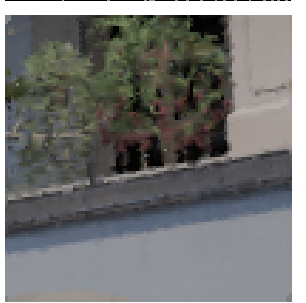

Recovery with $20 \%$ pixels PSNR: 31.86
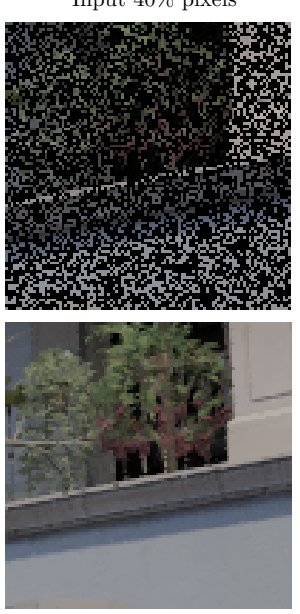

Recovery with $40 \%$ pixels
PSNR $: 33.46$

Figure 4.7: Examples of missing pixel recovery for sparsely rendered images with different sampling rates $(10 \%, 20 \%, 40 \%)$. 


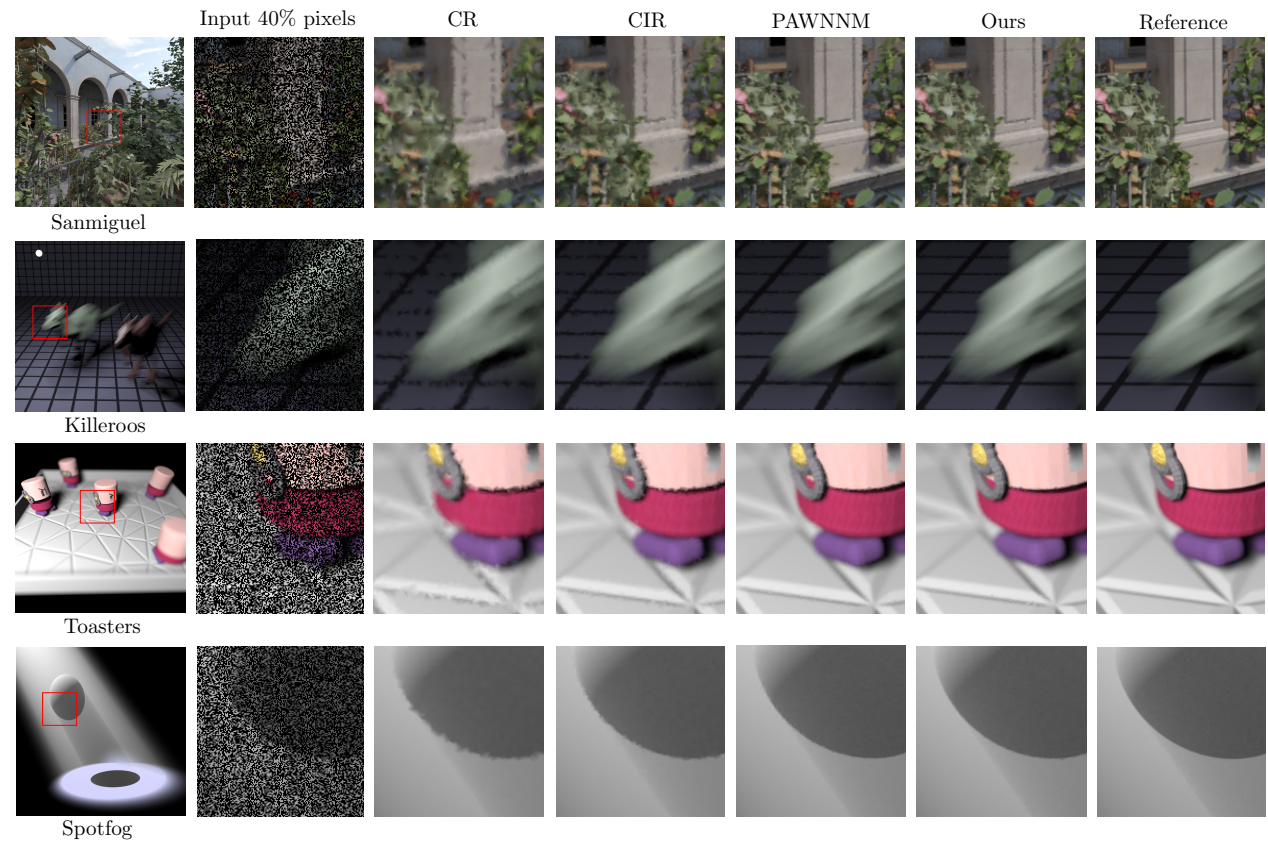

Figure 4.8: The recovery comparison with CR (Compressive Rendering), CIR (Compressive Image Reconstruction), PAWNNM, and our method.

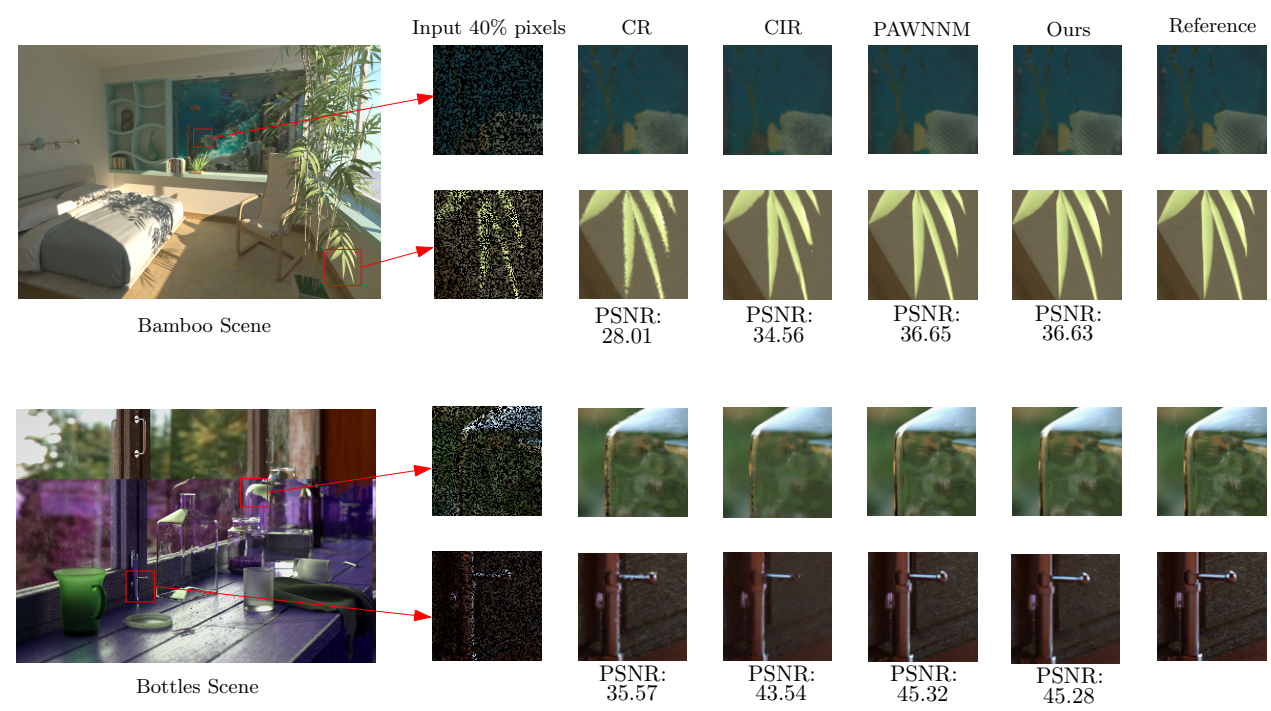

Figure 4.9: The recovery comparison of CR (Compressive Rendering), CIR (Compressive Image Reconstruction), PAWNNM, and our method. 

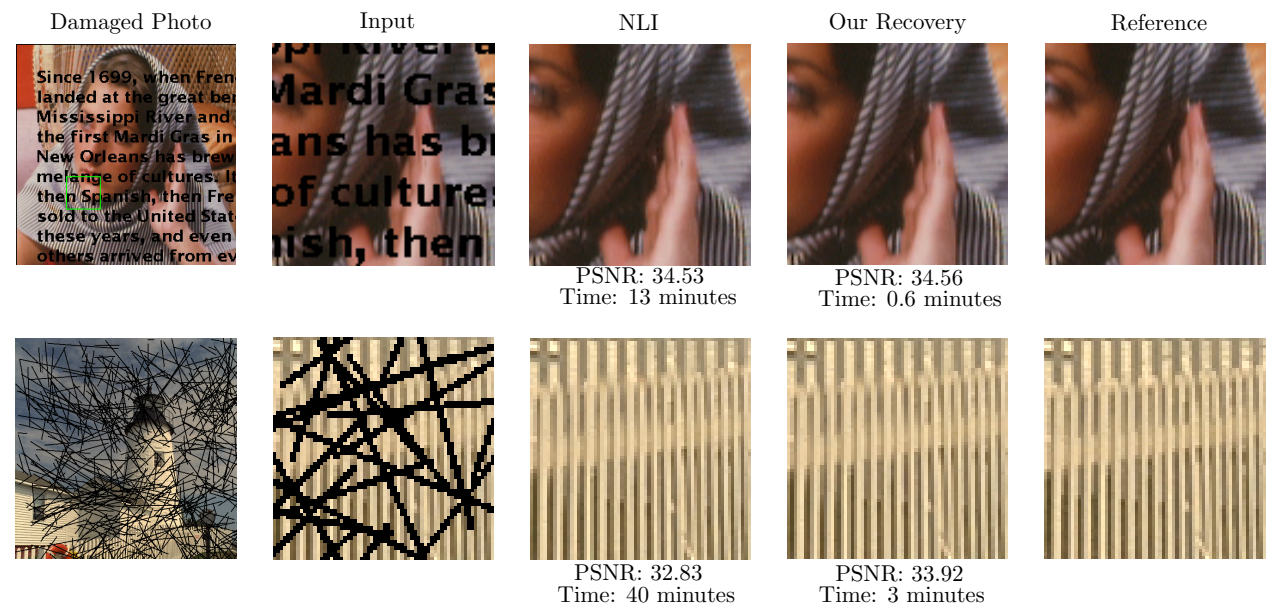

Figure 4.10: Examples of repairing damaged photographs. Compared with the previous method [4], our methods takes less time to recover the damaged pixels with slightly better quality.

in Algorithm (1) are also chosen experimentally, initialized as: $\beta=0.39$, $\omega=80.5$, and $c=0.9$. The number of iterations is set to 100 , where the recovery quality is progressively improved with more iterations. For example, using $40 \%$ sampling rate, we can observe convergence as it approaches 100 samples in Figure 4.6.

In Figure 4.4 we show that our BTM achieves a low rank matrix numerically without the need to use a complex initialization and patch finding algorithm as used in the previous methods [4, 101]. Figure 4.7 shows our recovery of images under $10 \%, 20 \%$ and $40 \%$ input pixels, where our method can generate plausible results for pre-visualization even with $10 \%$ input pixels.

We compare our method with state-of-the-arts approaches for path tracing pre-visulization, which include CR [9], CIR [23] using compressed sensing, and PAWNNM [101] based on matrix completion. We evaluated our method using test scenes that exhibit various properties, such as: a fine detailed structure, motion blur, soft shadow and depth of field. We show in Figure 4.8 that our method has better visual quality than CS based approaches, and similar visual quality as PAWNNM, but our method re- 
Table 4.1: PSNR/SSIM results of three test scenes under different input pixel sampling rates.

\begin{tabular}{lccccc}
\hline \multirow{2}{*}{ Scene } & Pixel portion & CR & CIR & PAWNNM & Ours \\
\hline \multirow{4}{*}{ Sanmiguel } & $60 \%$ & $29.43 / 0.912$ & $34.17 / 0.968$ & $35.95 / 0.975$ & $35.93 / 0.974$ \\
\cline { 2 - 6 } & $40 \%$ & $27.31 / 0.873$ & $31.93 / 0.947$ & $33.44 / 0.958$ & $33.46 / 0.961$ \\
\cline { 2 - 6 } & $20 \%$ & $25.66 / 0.852$ & $28.64 / 0.902$ & $30.58 / 0.916$ & $30.55 / 0.917$ \\
\hline \multirow{3}{*}{ Killeroos } & $60 \%$ & $32.40 / 0.949$ & $37.31 / 0.967$ & $38.68 / 0.975$ & $38.61 / 0.974$ \\
\cline { 2 - 6 } & $40 \%$ & $29.38 / 0.876$ & $33.46 / 0.951$ & $35.52 / 0.967$ & $35.59 / 0.969$ \\
\hline \multirow{3}{*}{ Toasters } & $20 \%$ & $26.71 / 0.822$ & $28.29 / 0.873$ & $31.96 / 0.926$ & $31.26 / 0.925$ \\
\cline { 2 - 6 } & $60 \%$ & $35.63 / 0.962$ & $39.42 / 0.978$ & $40.68 / 0.980$ & $40.63 / 0.979$ \\
\hline \multirow{3}{*}{ Spotfog } & $40 \%$ & $32.57 / 0.902$ & $36.58 / 0.961$ & $38.36 / 0.977$ & $38.34 / 0.975$ \\
\cline { 2 - 6 } & $20 \%$ & $28.92 / 0.875$ & $32.76 / 0.917$ & $35.64 / 0.963$ & $35.62 / 0.963$ \\
\cline { 2 - 6 } & $60 \%$ & $35.17 / 0.919$ & $43.23 / 0.975$ & $44.81 / 0.985$ & $44.79 / 0.984$ \\
\hline
\end{tabular}

quires less computation time. Rather than finding similar patches as done by PAWNNM [101], the BTM uses a numerical method using only the selected patch, which reduces the computation time. In our tests, the reconstruction time is reduced from 17 minutes (in [101]) to 8 minutes in a $1024 \times 1024$ image. We use the relative construction cost (reconstruction time/ partial rendering time) for evaluation in Table 4.2. Another two complex scenes that contain fine details are shown in Figure 4.9.

We also tested our method with inpainting applications, which often involves tasks such as photograph repair by removing scratches or overlayed text (Figure 4.10). Our method can effectively reconstruct the photographs which look similar to the original photographs. The previous method [4] took approximately 13 minutes to generate the repaired result, our method took only 36 seconds. 
Table 4.2: Recovery performance measured by relative recovery cost with respect to the total rendering time. The reference image is rendered in 32 hours.

\begin{tabular}{lccccc}
\hline Scene & Pixel Portion & CR & CIR & PAWNNM & EMR \\
\hline \multirow{3}{*}{ Samiguel } & $60 \%$ & $0.8 \%$ & $6 \%$ & $1.4 \%$ & $0.7 \%$ \\
\cline { 2 - 6 } & $40 \%$ & $0.8 \%$ & $5 \%$ & $1.4 \%$ & $0.7 \%$ \\
\cline { 2 - 6 } & $20 \%$ & $0.8 \%$ & $4 \%$ & $1.4 \%$ & $0.7 \%$ \\
\hline
\end{tabular}

\subsubsection{Multi-frame Recovery}

To evaluate our method for recovering the missing pixels from animation sequences, a video consisting of 150 frames is rendered for comparing with our previous methods PAWNNM in Chapter 3. In our test, we select ten frames as a tensor, and missing pixels values of the frames are recovered by the method described in Section 4.3.2; boundary frames of each tensor are overlapped and values are averaged.

Figure 4.11 shows the comparison with the patch based approach in Chapter 3. Our tensor completion based reconstruction can achieve better recovery quality than the patch based approach since we can consider the temporal coherence across frames with more global scene information rather than within a local region, and the temporal noise can be greatly reduced. We also demonstrate this by comparing the standard deviation of the recovery in Table 4.3. Benefiting from the tensor structure, the proposed method can escape the demanded of grouping similar patched from adjacent frames, our tensor-based multi-frame recovery also shows faster performance than the patch based approach (in Table 4.3). The total recovery time is only $6 \%$ of total rendering time. 


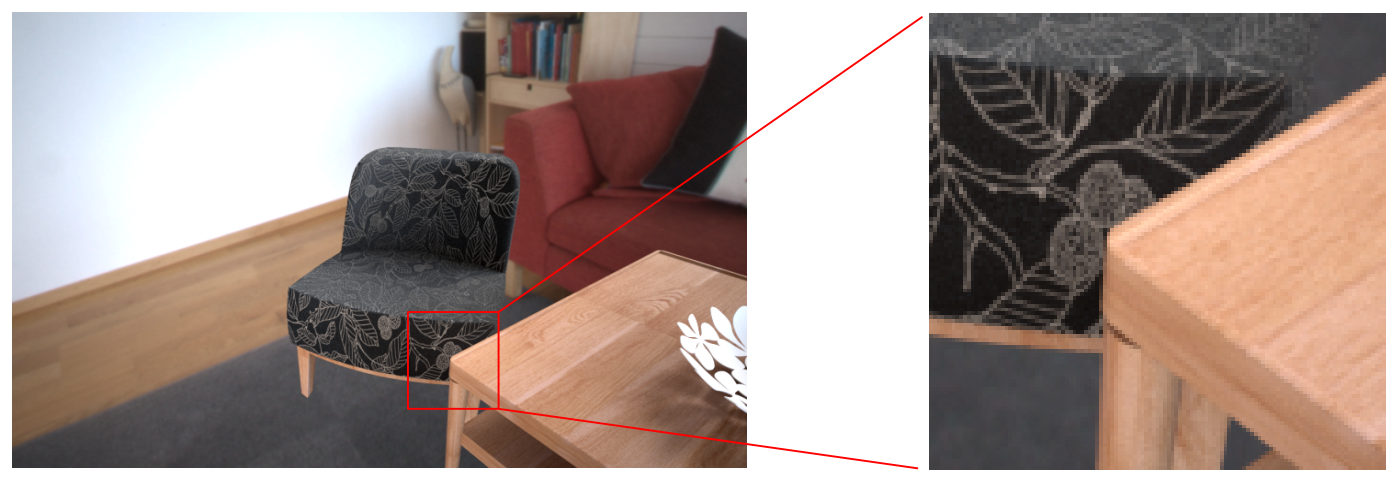

Reference

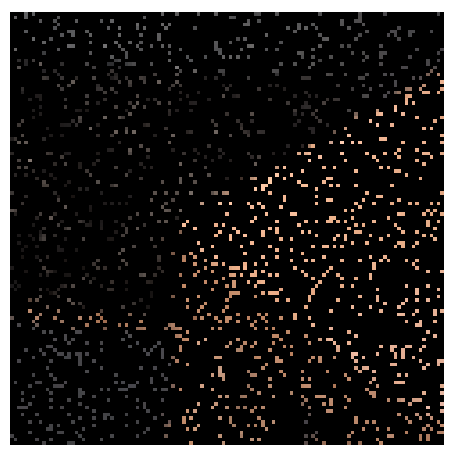

Input $10 \%$ pixels
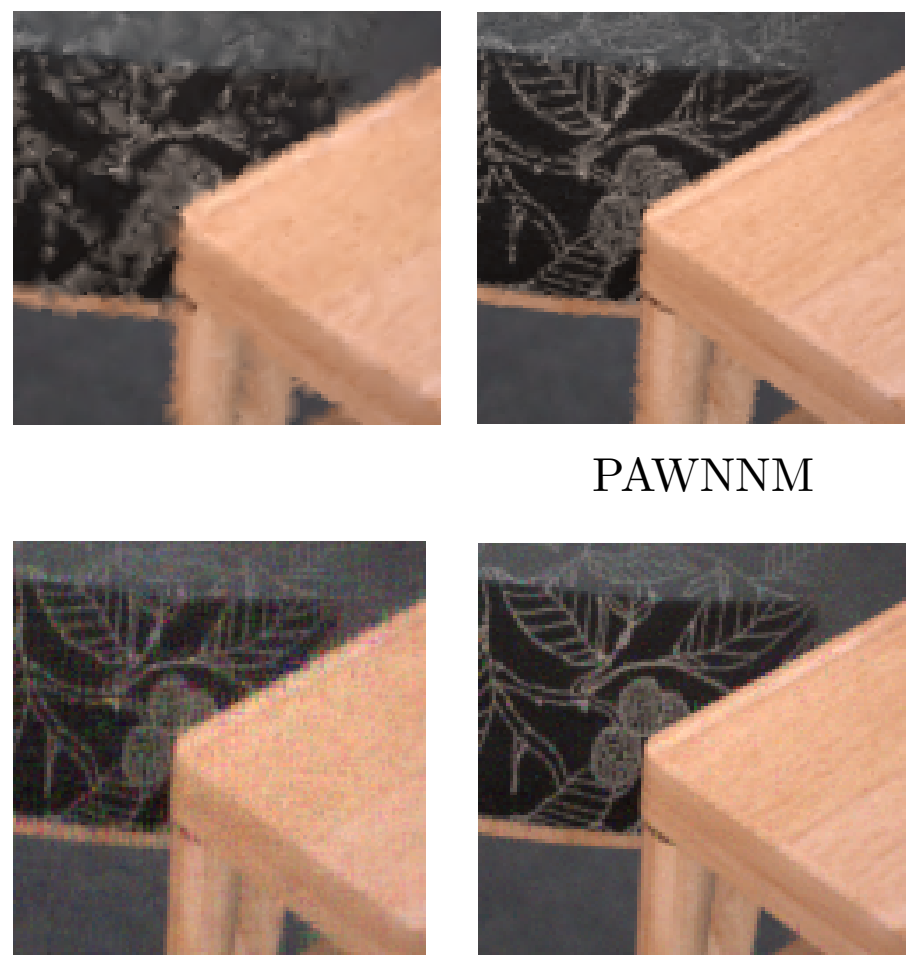
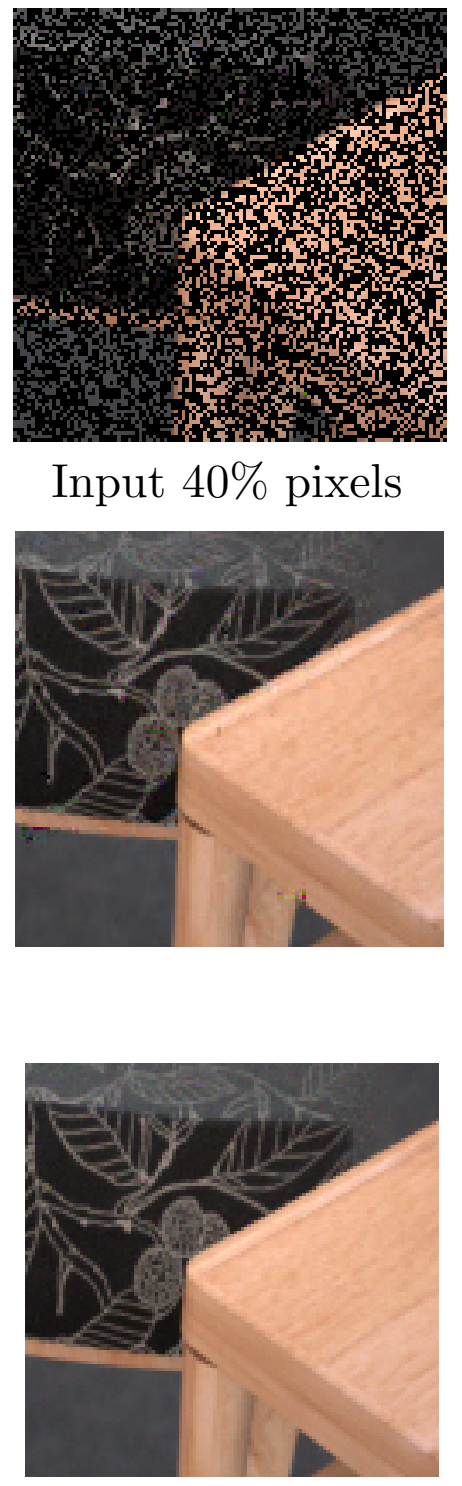

Input $40 \%$ pixels

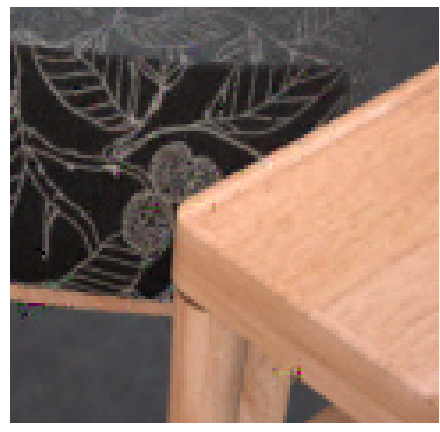

\section{PAWNNM}

\section{TCR}

Figure 4.11: Example of our proposed TCR and PAWNNM under different sampling rate. 
Table 4.3: Result of multi-frame recovery (with TCR): Incomplete video frames sampled at $10 \%, 20 \%$ and $40 \%$ are reconstructed independently (single-frame) and 10-frame concurrently (multi-frames). Time is measured in minutes.

\begin{tabular}{llllll}
\hline \multirow{2}{*}{$\begin{array}{l}\text { Input } \\
\text { Samples }\end{array}$} & $\begin{array}{l}\text { Recovery } \\
\text { Method }\end{array}$ & $\begin{array}{l}\text { Average } \\
\text { PSNR/SSIM }\end{array}$ & $\begin{array}{c}\text { Standard } \\
\text { Deviation }\end{array}$ & $\begin{array}{l}\text { Recovery } \\
\text { Time }\end{array}$ & $\begin{array}{l}\text { Full Render } \\
\text { Time }\end{array}$ \\
\hline \multirow{2}{*}{$10 \%$} & single-frame & $27.42 / 0.867$ & 1.62 & 1029 & \\
\cline { 2 - 5 } & multi-frames & $29.38 / 0.872$ & 1.03 & 86 & \\
\cline { 2 - 5 } $20 \%$ & single-frame & $31.74 / 0.905$ & 1.25 & 1029 & \multirow{2}{*}{1440} \\
\cline { 2 - 5 } & multi frame & $34.23 / 0.931$ & 0.80 & 86 & \\
\cline { 2 - 5 } $40 \%$ & single-frame & $35.17 / 0.934$ & 1.08 & 1029 & \\
\cline { 2 - 5 } & multi frame & $39.41 / 0.956$ & 0.42 & 86 & \\
\hline
\end{tabular}

\subsection{Summary and Discussion}

In this chapter, we have presented two efficient solutions for pixel recovery and multi-frame recovery. We first applied an enhanced matrix completion based on Toeplitz structure to reconstruct the missing pixels in a single rendered image. To exploit the low-rank property for the recovery of the missing pixel samples in an enhance matrix structure, we employed the ADMM algorithm with a parameter adjustment strategy that alternatively estimate the factorization matrices. Since our algorithm is SVD free, the computational time is faster than the approach presented in Chapter 3.

We then introduced tensor structure and apply tensor completion for recovering missing values of partially rendered animation sequences. We exploited the tensor singular value decomposition, which can characterize information and structural complexity of multi-frame rendering. By applying the PAWNNM to solve the tensor completion problem, our multiframe recovery shows significant performance gain compared to the proposed patch based reconstruction in the previous chapter. The primary 
contribution of the work presented in this chapter is the introduced enhanced matrix completion with Toeplitz structure, which helps to significantly reduce the overall recovery time compared to the pixel recovery in Chapter 3. Additionally, by adapting the tensor structure to multi-frame restoration, global coherence information can be explored across both the image domain and time domain. The proposed multi-frame reconstruction solution can generate a more accurate result within less time than the proposed technique in Chapter 3. Finally, by incorporating the progressive adjustment strategy with weighted nuclear norm minimization into standard enhanced matrix completion and tensor completion, our proposed strategy can achieve better recovery quality.

The proposed reconstruction methods have similar limitations with the methods in Chapter 3. One limitation is that the proposed reconstruction methods are challenged when attempting to recover pixel values under very low sampling rates $(<10 \%)$. Another limitation of the reconstruction methods is the requirement of rendering captured pixels with sufficient samples, which means an extra time is spent before the post process. Differently, a denoising based method only requires a few samples to generate the input. Thus, it seems more efficient than a reconstruction based manner. In next chapter, we will introduce a novel denoising filter approach to efficiently achieve noise-free path tracing. 
82 CHAPTER 4. ENHANCED COMPLETION FOR PATH TRACING 


\section{Chapter 5}

\section{Denoising Network for Path Tracing}

As introduced in Chapter 1, rendering photorealistic images of a virtual scene requires simulating all the possible light paths between the light sources and view camera. Path tracing [5] provides a general approach to approximating this problem. The method has been widely applied in feature film and visual effects production since it can meet the high-quality requirement of the industry. Unfortunately, this task involves generating a large number of samples and lengthy computation time for noise-free renderings.

To mitigate this problem, previous two chapters have investigated the reconstruction based approaches to provide efficient and accurate solutions for path tracing which provides the ability to recover the missing samples based on rendered ones, but the reconstruction methods require rendering the captured pixels or sub-pixels with sufficient samples, which means less efficiency under a low sample budget. We will now turn to the denoising based approach. A large number of works have been proposed to generate a noisy rendering with a low sample budget quickly and in the process the application of a denoising filter to output a noise-free result $[10,11,12,13,14,15]$. These methods usually apply a scene feature 
based filter, like non-local mean filter and bilateral filter, which can utilize additional scene feature buffers (e.g. world position, depth information) to guide the denoising process. Due to the additional scene information is closely related to scene details; these features based denoising filters achieve a good quality result. But the main challenge is how to set the filter weights for different features properly. Many approaches use error estimation metrics to select the best filter parameters. Since the error metrics are noisy under a low sample budget, the accuracy of the selected parameters are not the optimum ones. Therefore, the denoised results from these methods may suffer from over blurred areas, especially in the region with high-frequency details.

More recently, the convolutional neural network has been applied and achieved better performance than the feature based denoising filters. Kalantari et al. [80] introduced a multi-layer perceptron learning-based model. A recurrent denoising autoencoder is proposed to reconstruct rendered animation sequences [83]. Also, kernel predicting based convolutional network is proposed to train and evaluate with production data [81]. The CNN based denoising network can learn the complex relationship between noisy rendering and corresponding ground-truth by leveraging both the color values and the auxiliary scene features, while showing state-ofthe-art performance. Once the neural network is trained, it can be easily and efficiently applied without complex parameter selection. Such a framework can more efficiently handle the task of previsualization of path tracing.

Motivated by these deep learning based works, in this chapter, we propose to apply a multi-scale residual learning based denoising network. The basic task is to render a noisy result in a quick manner with only a few samples per pixel, and then apply the denoising network to output a noise-free result within several seconds, which is more efficient than the reconstruction methods. Unlike the previous $\mathrm{CNN}$ based architectures, which apply a single scale filter in the convolutional layer or perform 
multi-scale concept through down/up-scale feature maps, we apply different filters during the convolution step to preserve both long and short contextual information. Additionally, we propose a separated residual learning strategy to predict the noise and generate a virtual flash image as one of the input scene feature buffers.

\subsection{Residual Learning for Denoising Path Trac- ing}

Denoising in image processing mostly challenges spatially-inveriant Gaussian noise [78], which means evenly and globally distributed noise in an image. Figure 5.1 shows an example of path tracing image generated by limited samples per pixel. As shown in Figure 5.1, since the different local regions shows inhomogeneous noise in a image, conventional denoising in image processing is not efficient.

Although the noise level in path tracing is non-uniform and typically does not follow a Gaussian distribution over the whole image space, we observed that the residual noise within a local area is nicely approximated by a Gaussian distribution within the local area. As shown in Figure 5.1, the histograms of residual noise in different patches are near Gaussian distributions. Therefore, we show that this consistent representation of the residual is learned by the neural network more efficiently.

\subsection{Dual Channel Residual Learning}

Path tracing is a process to evaluate the light paths from camera to light sources by randomizing the direction of the ray at the surface intersection though the entire scene. During rendering, each sample ray within a pixel may not always successfully find a complete light path and contribute to calculating the final pixel value. Therefore, noise artifacts are caused by 


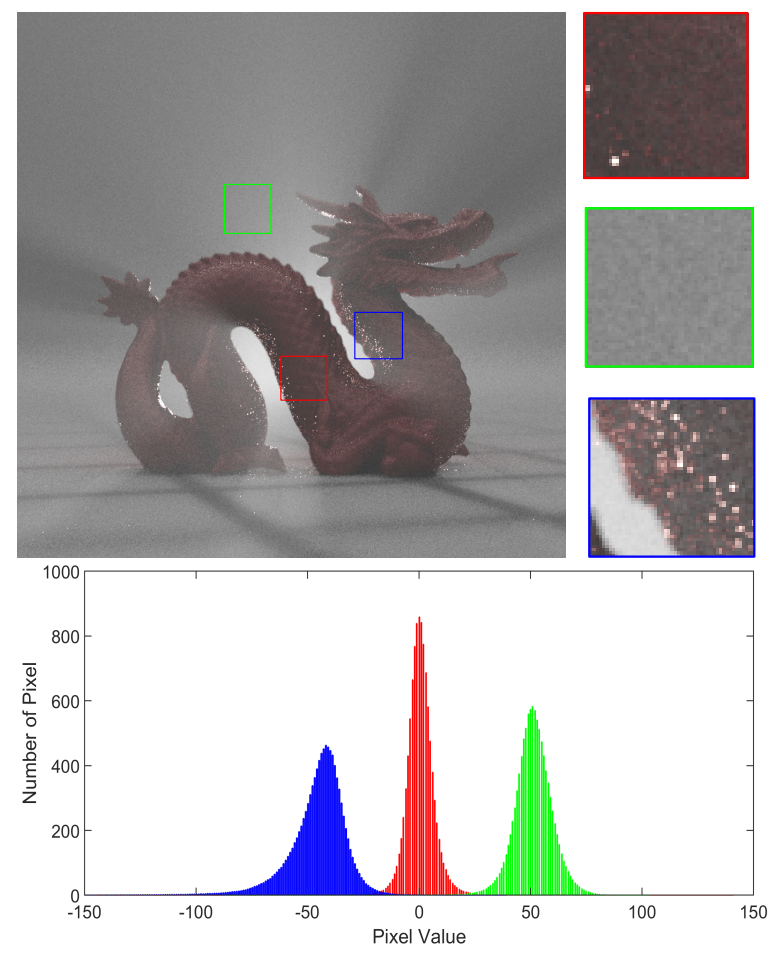

Figure 5.1: In a noisy path tracing (the top), the noise level is various in different image regions; the noise level in the region of blue window is higher than the region of red window. In contrast, the area in the green window contains less noise. The bottom figure is the histogram distributions of the residual from the three different patches, showing near Gaussian distributions.

insufficient numbers of complete light paths during the rendering phase. Pixel value integrated by the insufficient samples would be either over or under exposed than the reference image. In Figure 5.2, the scene is rendered with 8 samples per pixel (spp), some pixels in the noisy rendering are much brighter or darker than the corresponding pixels in the reference image. For example, the pixel in the green square obviously has a higher value than the reference rendering, while the pixel value in the yellow square looks lower. 


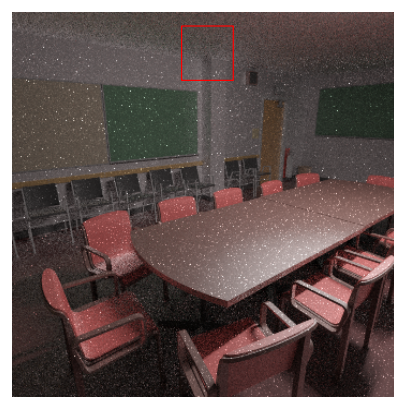

Classroom

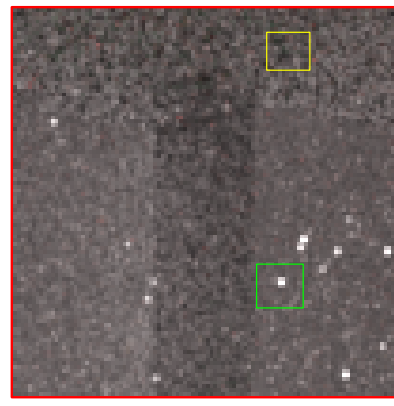

Input $8 \mathrm{spp}$

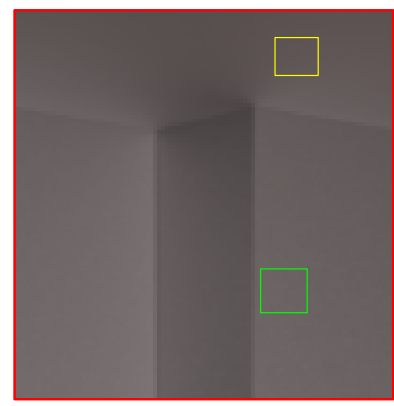

Reference

Figure 5.2: An example of noise artifacts in image rendered with only 8 samples per pixels (left image). The noise artifacts are caused by either extreme larger (green square) or smaller (yellow square) pixel value compared to the corresponding pixel in the reference rendering.

Given a noisy rendering $y$ and a corresponding reference image $G$, we define the over and under exposed signals in the noise rendering as bright channel residual $R_{b}$ and dark channel residual $R_{d}$ respectively:

$$
\begin{gathered}
R_{b}=\left\{\begin{array}{cc}
y_{i}-G_{i}, & y_{i} \geq G_{i} \\
0, & y_{i}<G_{i}
\end{array}\right. \\
R_{d}=\left\{\begin{array}{cc}
G_{i}-y_{i}, & G_{i} \geq y_{i} \\
0, & G_{i}<y_{i}
\end{array}\right.
\end{gathered}
$$

where $i$ is the pixel position in the rendering.

The objective of our method is to remove the noise artifacts from the image $y=\left\{y_{r}, y_{g}, y_{b}\right\}$ rendered with a few samples per pixel (e.g., $8 \mathrm{spp}$ ), while aiming to have the denoised output with visual quality as close as possible to the reference image $G$ that rendered with many samples (e.g., $5 \mathrm{k}$ to $64 \mathrm{k} \mathrm{spp}$ ). We apply a convolutional neural network to learn the mapping $g$ between the noisy input and the reference image. Given the inputs of network include a noisy rendering $y$ and a set of feature images $f$ (i.e. surface normal, depth information, virtual flash image), our learning pro- 
cess can be described as:

$$
\Theta^{*}=\underset{\Theta}{\arg \min } \ell(G ; g((y, f) ; \Theta))
$$

where $\ell$ is an error metric between the denoised result and the input noisy rendering. $\Theta$ is network parameters. $\Theta^{*}$ is the optimum ones we aim to estimate during the training of the network. We use the residual learning technique to learn a mapping of the residual:

$$
R(y, f) \approx G-y
$$

Specifically, we separate the residual $R$ into two channels: bright channel residual $R_{b}$ and dark channel residual $R_{d}$. By applying the separated networks to predict the two channels' residuals, we show that dual channel residual learning can boost the training process to learn efficient denoising filter for path tracing. Therefore, based on Eq.5.3, learning process would be:

$$
\begin{aligned}
\Theta^{*} & =\underset{\Theta}{\arg \min } \ell(G ; y+R(y ; \Theta)) \\
& =\underset{\Theta_{b, d}}{\arg \min } \ell\left(G ; y+R_{b}\left(y ; \Theta_{b}\right)+R_{d}\left(y ; \Theta_{d}\right)\right)
\end{aligned}
$$

where the network parameters $\Theta$ can be expressed into two parts: $\Theta_{b}$ and $\Theta_{d}$, which represent the parameters for $R_{b}$ and $R_{d}$ respectively.

\subsection{Multi-scale CNN}

We present a novel multi-scale CNN for dual channel residual $\left(R_{b}\right.$ and $R_{d}$ ) learning. In order to extract features from the input patches (the noise and scene feature images), multi-scale kernels are developed in each convolutional layer. Compared with the conventional CNN using fixed size kernels, our multi-scale CNN provides more robust path tracing denoising for complex scene with various contextual details. 


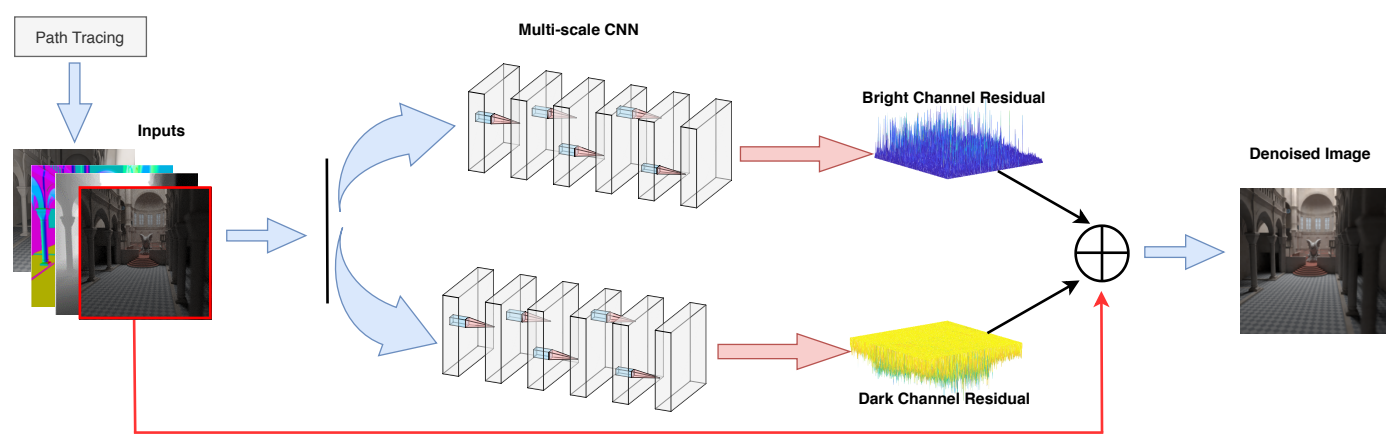

Figure 5.3: Overview of the proposed denoising pipeline. The input of the network consists of a noise rendering, normal vector, depth information and virtual flash image buffer. The input is fed to two residual networks for prediction of the bright and dark channel residual separately. The denoised image is achieved by combining the residual images with the noise rendering.

\subsubsection{Network Architecture}

Two separated CNNs were set up to predict the bright channel residual and the dark channel residual. Figure 5.3 illustrates the overall flow of our denoising pipeline. As shown in the overall architecture of our network (Figure 5.3), the bright and dark channel residual networks have the same structure, therefore we only explain details for the bright channel residual.

The inputs to the network are patches of a noisy rendering and corresponding scene features (normal vector, depth, virtual flash image). For a $N$-layer CNN, the first $N-2$ layers include a $n$ types of multi-scale filters for multi-scale convolution. The multi-scale convolutional neural network has been applied for object detection [102], medical image processing [103]. However, it has not been applied to solve path tracing denoising. We use the Maxout function [104] as the activation function. For layer $(N-1)$, a single linear convolution is applied, and then rectified linear units (ReLU)[105] is used as the activation function. The last layer contains a single scale convolution to reconstruct the denoised output. 


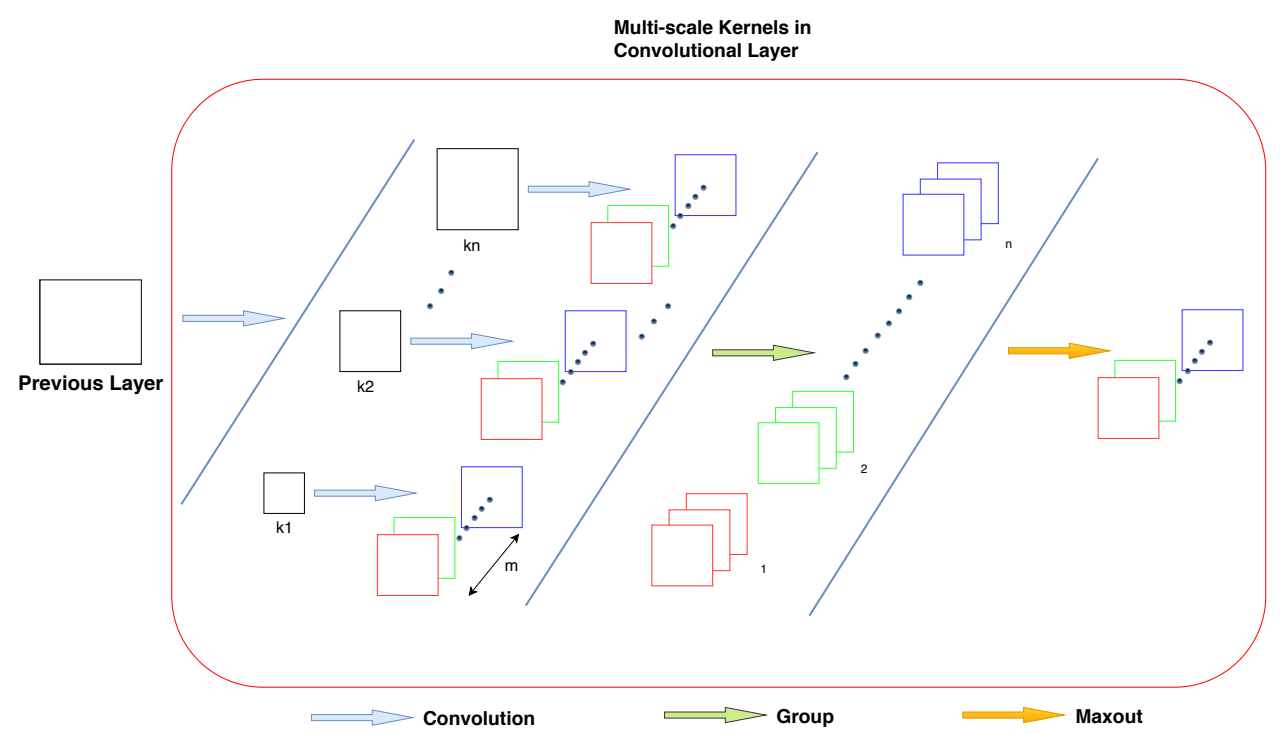

Figure 5.4: The multi-scale structure in the convolutional layer of our network. Outputs from the previous layer are convolved with different size filters to generate new features maps. Then, group the new feature maps using Maxout activation to generate the outputs feature maps.

We presents a novel concept that present a multi-scale filters inside each convolutional layer rather than using the multi-scale strategy for resizing input data $[102,103]$. Since the important details of the inputs could be lost through multi-scale resizing, our multi-scale filters operate inside the convolutional layers extracting the feature details from the different sized kernels. An optimal selection for denoising filter is to use a small scale filter for high frequency area, and a large scale filter for the smooth area.

As illustrated in Figure 5.4, supposing there are $n$ types of filter $k_{i}$ in the multi-scale convolutional layer, and each type of filter contains $m$ filters. Thus, each type of filter generates $m$ feature maps, and there will be $n \times m$ feature maps in total. Then, all feature maps will be separated into $m$ groups. 


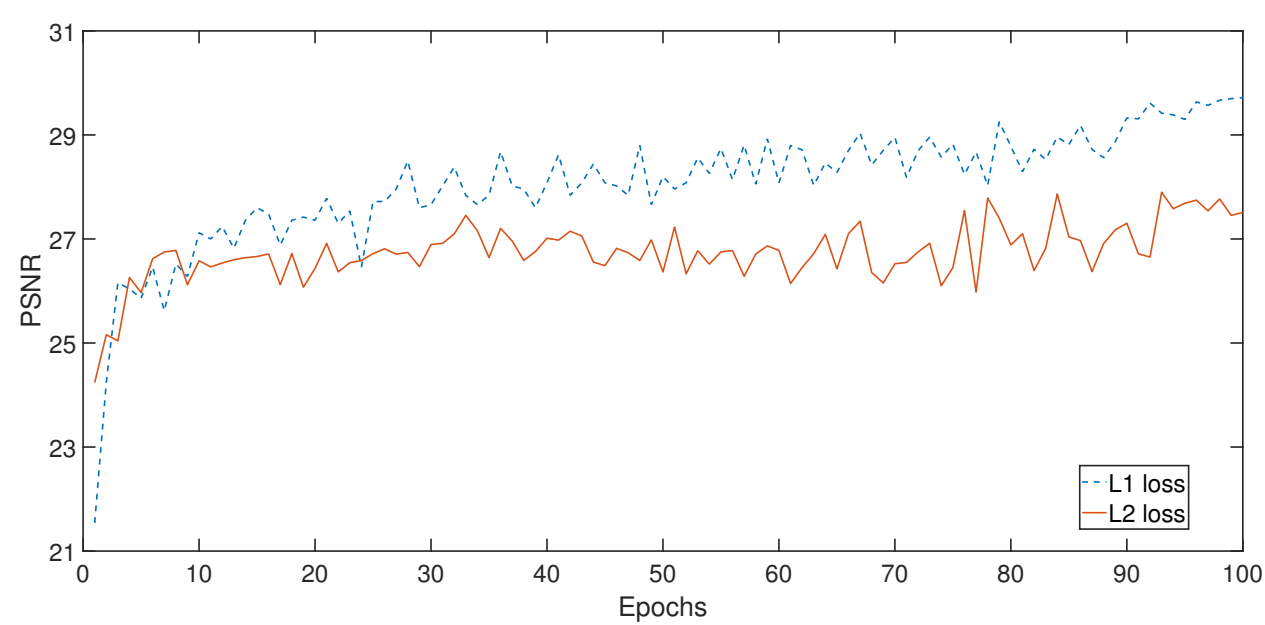

Figure 5.5: Evaluation of the learning performance with different loss functions. The network is trained with $L_{1}$ loss and $L_{2}$ loss separately to compute the average PSNR (peak signal-to-noise ratio) on 7 tested images. With a comparison, $L_{1}$ loss is more efficient than $L_{2}$ loss.

\subsubsection{Loss Function}

To estimate the error between the outputs from the CNN and target reference images, a loss function is calculated while training the network. In image processing, $L_{2}$ loss is generally applied, to computes the mean squared error between the predicted and the target reference image. However, we apply $L_{1}$ loss as our loss function:

$$
L_{1}=\frac{1}{N} \sum_{i}^{N}\left|D_{i}-R_{i}\right|
$$

here, $D_{i}$ and $R_{i}$ represent the $i$ th pixel in the denoised and reference image respectively.

$L_{1}$ loss can better mitigate the splotchy artifacts in the denoised image comapre with $L_{2}$ loss [106]. Figure 5.5 shows the average PSNR estimated using $L_{1}$ and $L_{2}$ loss function. After 10 epochs, the network trained by the $L_{1}$ loss shows a better result than the one using $L_{2}$ loss. The bright and dark channel residual networks are trained independently with $L_{1}$ loss in 
our networks. Figure 5.7 shows the training error of the proposed denoising network.

\subsubsection{Training Data}

The training data set in our experiment consists of $140 \mathrm{~K}$ patches for each input including noise colour, normal, depth, and virtual flash images. All data has been generated from 30 scenes that contain various effects such as motion blur, global illumination, and depth of field. To increase the diversity of the training dataset, each scene is rendered with 4, 8, 16, 32, 64 spp. The reference images are rendered with a huge number of spp (e.g. $5 \mathrm{k}$ to 64k). Additionally, each scene is rendered four times at each sampling rate to avoid overfitting to specific noise patterns. After generating the training data, the images are cropped into $65 \times 65$ patches. During training, rotation and flip operations are randomly applied to each training patch pair.

The input is generated using PBRT [107]. Besides these commonly used scene features (noise colour, normal, depth), we utilized a virtual flash image as an additional input.

\subsubsection{Virtual Flash Image}

We utilize a virtual flash image as the additional scene feature in our approach in order to provide various important details in the scene; benefits of using a flash image has been demonstrated in few previous denoising methods $[108,109,110]$. An example of our virtual flash image is shown in the top row in Figure 5.6. To generate the virtual flash image, a virtual point light is set up at the camera position and then the rendering image is generated without original light sources capturing the high-frequency scene details. Figure 5.6. (bottom row) shows the pixel histogram distributions of the reference rendering, virtual flash image, and noisy rendering 


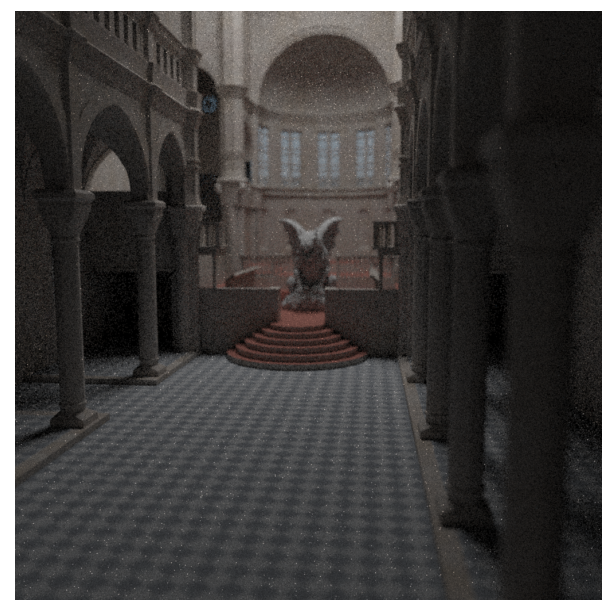

Noisy Image

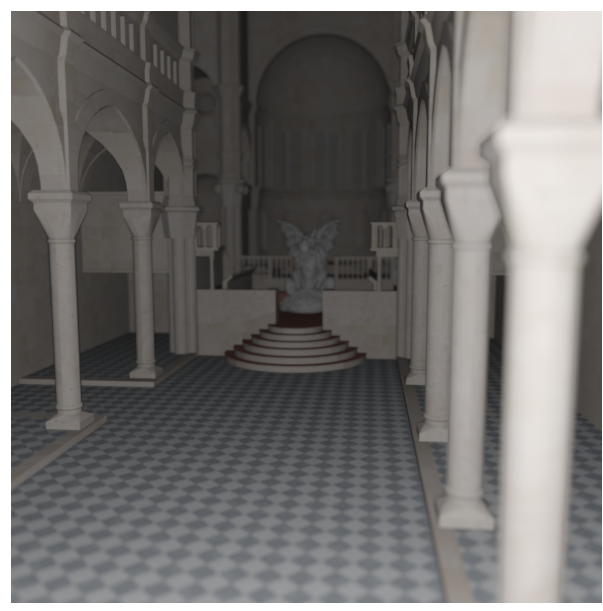

Virtual Flash Image

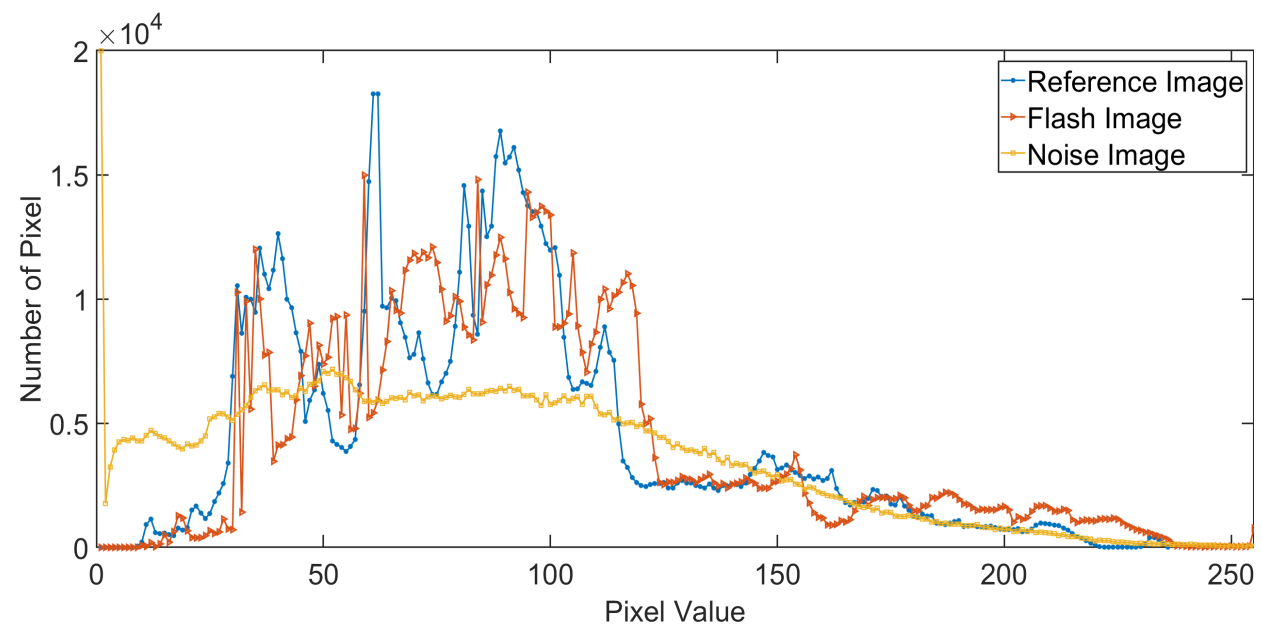

Figure 5.6: Top row (from the left): a noisy rendering produced with limited samples, and the corresponding virtual flash rendering. Bottom row: histograms of the reference, noise, and virtual flash image. 
Table 5.1: Denoising results of multi-scale CNN in different kernel size.

\begin{tabular}{cc}
\hline Multi-scale Kernel Sizes & PSNR \\
\hline$<3,5,7>$ & 27.68 \\
$<3,5,9>$ & 29.24 \\
$<5,7,9>$ & 28.12 \\
$<5,9,13>$ & 27.83 \\
$<7,9,13>$ & 24.56 \\
\hline
\end{tabular}

of the scene. The virtual flash image could better approximate the distribution pattern of the ground truth rendering. Also, the virtual flash image can depict scene features where the shadow area is under the original light sources. Additionally, rendering the virtual flash image is quick, since we only consider the direct illumination without additional ray samples. The virtual flash feature image contributes to preserve high-frequency information in our denoised results.

\subsubsection{Training Details}

For the bright and dark channel residual networks, we apply six convolutional layers with 64 filters in each layer expect the final layer, which contains three kernels. From the first layer to the fourth layer, three different size kernels are used: $3 \times 3,5 \times 5$ and $9 \times 9$ respectively. We denote the setting of these kernel sizes as $<3,5,9>$. The kernel size for the fifth layer and the last layer is set to $5 \times 5$ and $3 \times 3$ respectively. The kernel size of the multi-scale convolutional layer have been experimentally selected. As shown in Table 5.1, we modify the multi-scale kernel size into other options (e.g. $<5,7,9>$ ) and compute the average PSNR on seven tested scenes. Our selection of $<3,5,9>$ achieves best performance. For 


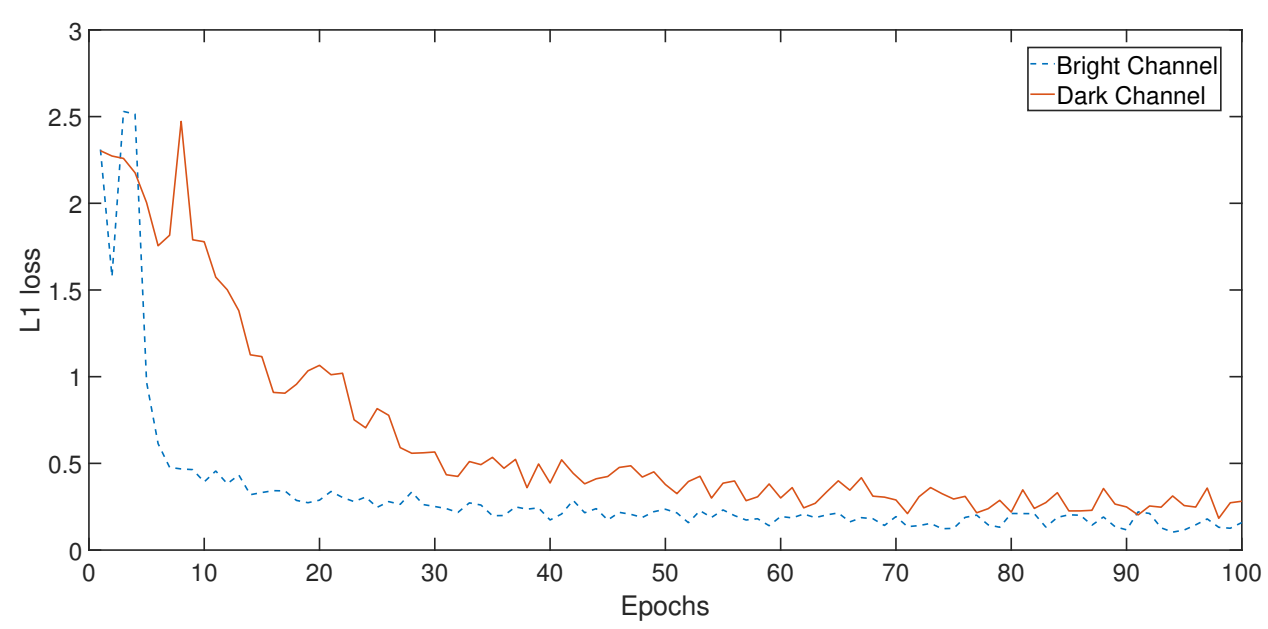

Figure 5.7: Training error of the proposed bright and dark channel network trained on $L_{1}$ loss function.

efficient network training, mini-batch training is used, which uses a subset of the training data during computing the back-propagation of each iteration. The size of the mini-batch is set to $64 \mathrm{spp}$ for our training. As common setting with other deep learning methods, we chose Adam solver [111] as optimizer in TensorFlow [112] with a learning rate of 0.0001, and trained on NVIDIA GTX1080 graphics card. We trained 100 epochs for two networks, the training time is around 3 days.

\subsection{Results}

\subsubsection{Denoising Performance}

This paper presents a series of contributions to enhance denoising quality for the path tracing image using CNN. These include dual channel residual learning, multi-scale CNN, and an additional scene features using the virtual flash image. We tested our result in several CNNs with different combinations to clarify the performance gain from each contribution. The first step is to show the performance difference between single and dual 


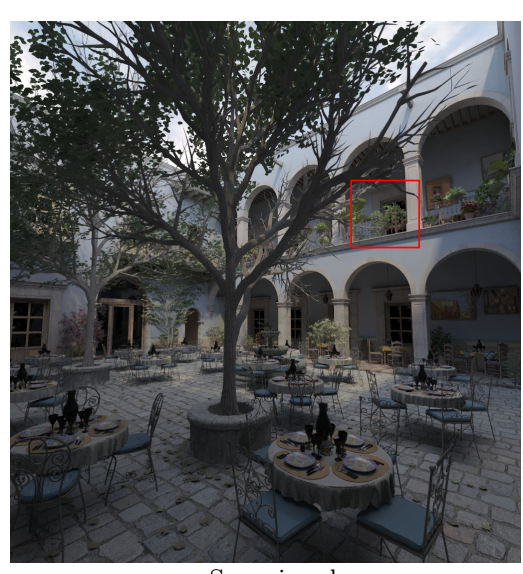

Sanmiguel
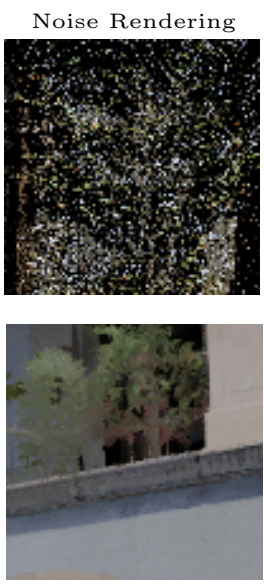

DRM
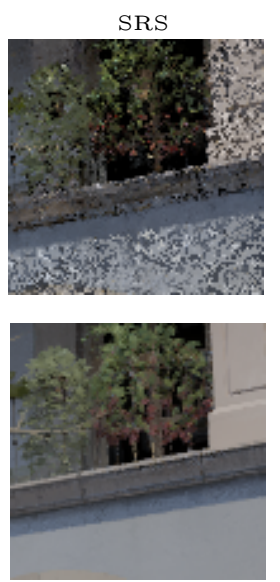

DRMF
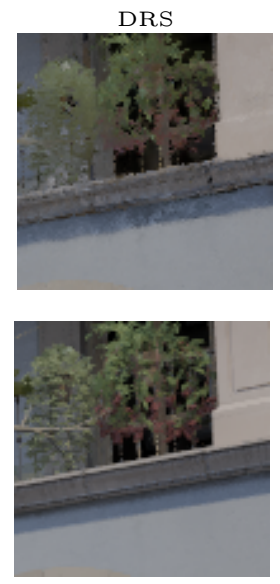

Reference

Figure 5.8: We introduce dual channel residual learning with multi-scale convolutional neural networks for denoising path tracing renderings. We trained networks for single channel residual learning (SRS) and dual channel residual learning (DRS) using a single fixed scale CNN, a dual channel residual using a multi-scale CNN with (DRMF) and without the virtual flash image (DRM). The noisy input is rendered with 32 spp (samples per pixel), while the reference image is rendered with 64k spp. Our proposed DRMF closely resembles the reference.

channel residual learning. We trained networks for a single channel residual learning (SRS) and a dual channel residual learning (DRS) using a single fixed scale CNN. Then, we trained a network to learn the dual channel residual using a multi-scale CNN with (DRMF) and without the virtual flash image (DRM).

Figure 5.8 shows the results of each setup. Although SRS shows good denoising for the changeling scene generated by highly limited samples, it shows various artefacts including over/under exposed noisy signals. Our dual channel residual learning (DRS) shows outstanding performance to denoise over/under exposed noisy signals. Then, our multi-scale CNN (DRM) further improve denoising in the low frequency area while preserving the high frequency details. Finally, our virtual flash image fea- 
ture (DRMF) contributes to enhance overall denoising performance while adding extra scene details limited by the noisy rendering input. Our DRMF shows outstanding performance to denoise path tracing image even for the very challenge case as shown in Figure 5.8 (a noisy complex scene rendered by a highly limited number of samples). The final result of our denoising for the noisy rendering with limited samples (32 spp) shows comparable visual quality with the reference rendering having $64 \mathrm{~K} s p p$.

\subsubsection{Performance Comparison}

We compared our DRMF with state-of-the-art denoising methods for path tracing. Our method was compared with two recent learning-based methods using the neural networks, LBF [80] and KPCN [81]. Then, compare our DRMF with colour based denoising, RHF [113], and scene feature based denoising, NFOR [114]. All methods are based on the original implementation with default settings described by the papers. Two most commonly used error metrics PSNR and SSIM are used to evaluate the denoising result of the noisy image (rendered by 8 to $32 \mathrm{spp}$ ) with respect to the reference image (rendered by $5 \mathrm{k}$ to $64 \mathrm{~K} \mathrm{spp}$ ).

Denoising comparison with CNN based methods: Figure 5.9 shows comparison results with the CNN based approaches, LBF and KPCN. We tested across four challenging scenes such as Kitchen, Livingroom1, Living room 2 and Bathroom (noise image with $8 \mathrm{spp}$, reference image with 64k spp). Our method (DRMF) shows better result compared with LBF and KPCN. In particular, our method outperform in the selected regions; both LBF and KPCN shows artefacts such as over blurring the high frequency areas (taps of the Bathroom scene) or under smoothing for low frequency areas (table of the Livingroom 2). Table 5.2 shows the error metrics (PSNR and SSIM) of the entire image to compare denoising results of our methods (SRS, DRS, DRM, and DRMF) with others.

Denoising comparison with colour/feature based methods: Figure 


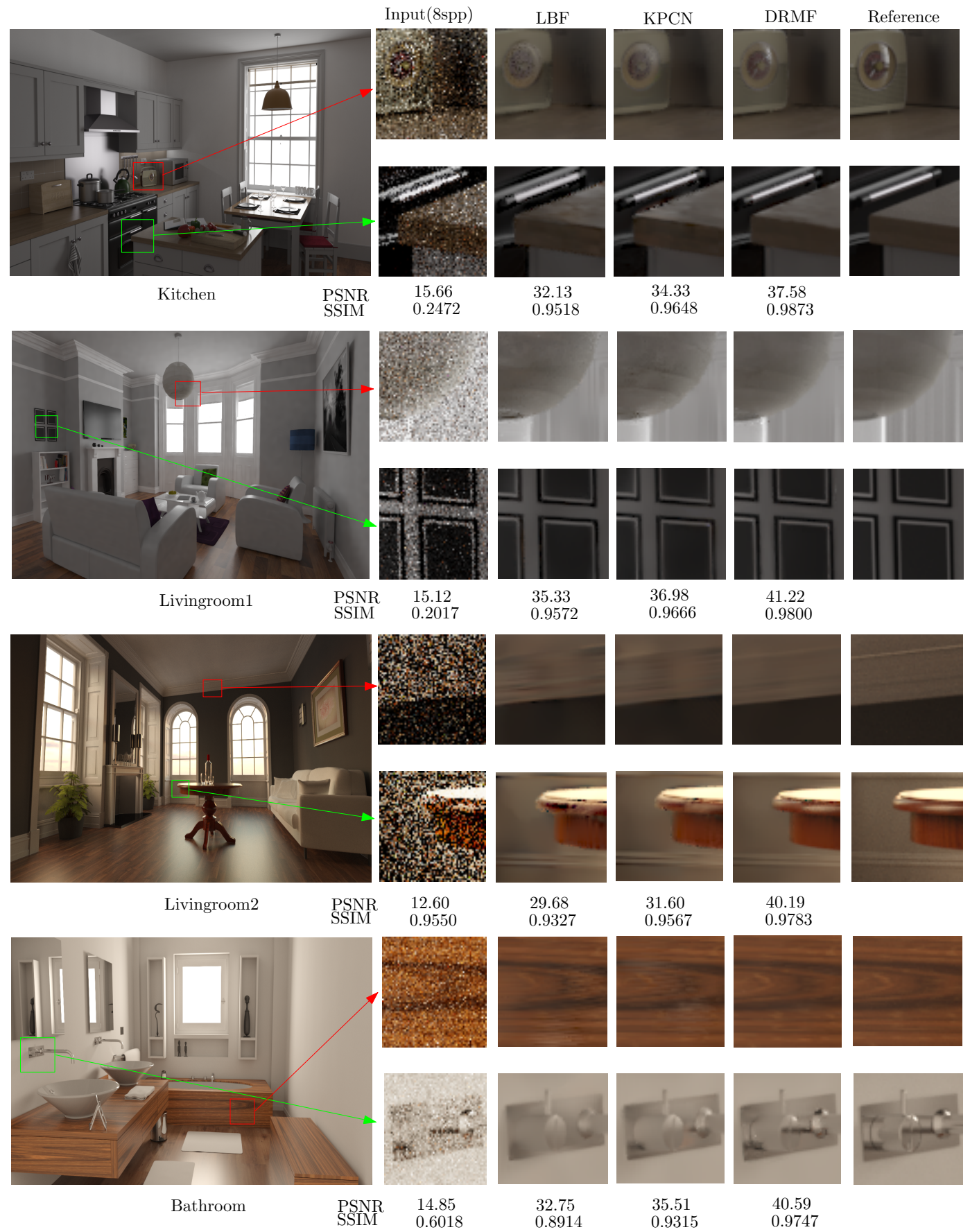

Figure 5.9: Comparison of LBF, KPCN and our proposed denoising network (DRMF). 


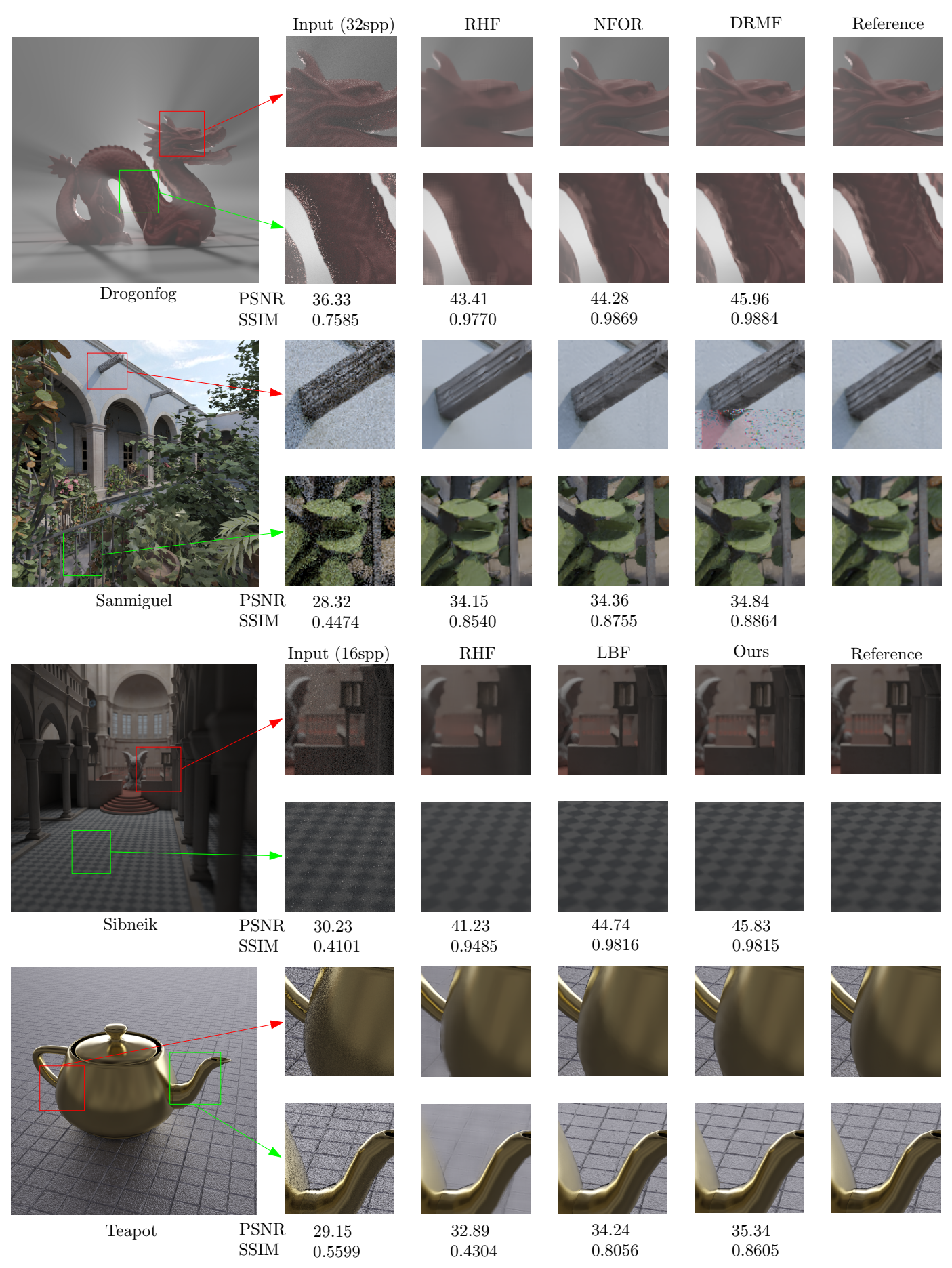

Figure 5.10: A visual quality comparison of RHF, NFOR and our proposed denoising network (DRMF). 
Table 5.2: PSNR/SSIM results of our methods (SRS, DRS, DRM, and DRMF), LBF and KPCN.

\begin{tabular}{ccccccc}
\hline & SRS & DRS & DRM & DRMF & LBF & KPCN \\
\hline Kitchen & $29.86 / 0.9316$ & $30.59 / 0.9458$ & $31.79 / 0.9502$ & $\mathbf{3 7 . 5 8 / 0 . 9 8 7 3}$ & $32.13 / 0.9518$ & $34.33 / 0.9648$ \\
\hline Livingroom1 & $30.38 / 0.9326$ & $34.84 / 0.9511$ & $35.17 / 0.9525$ & $\mathbf{4 1 . 2 2 / 0 . 9 8 0 0}$ & $35.33 / 0.9572$ & $36.98 / 0.9666$ \\
\hline Livingroom2 & $28.04 / 0.9035$ & $28.39 / 0.9248$ & $29.17 / 0.9325$ & $\mathbf{4 0 . 1 9 / 0 . 9 7 8 3}$ & $\mathbf{2 9 . 6 8 / 0 . 9 3 2 7}$ & $31.60 / 0.9567$ \\
\hline Bathroom & $26.59 / 0.8426$ & $28,84 / 0.8639$ & $31.24 / 0.8857$ & $\mathbf{4 0 . 5 9 / 0 . 9 7 4 7}$ & $32.75 / 0.8914$ & $35.51 / 0.9315$ \\
\hline
\end{tabular}

Table 5.3: PSNR/SSIM results of our methods (SRS, DRS, DRM, and DRMF), RHF and NFOR.

\begin{tabular}{ccccccc}
\hline & SRS & DRS & DRM & DRMF & RHF & NFOR \\
\hline Dragonfog & $40.15 / 0.9463$ & $41.86 / 0.9636$ & $43.07 / 0.9731$ & $\mathbf{4 5 . 9 6 / 0 . 9 8 8 4}$ & $43.41 / 0.9770$ & $44.28 / 0.9869$ \\
\hline Sanmiguel & $32.96 / 0.8357$ & $33.12 / 0.8401$ & $33.24 / 0.8479$ & $\mathbf{3 4 . 8 4 / 0 . 8 8 6 4}$ & $34.15 / 0.8540$ & $34.36 / 0.8755$ \\
\hline Sibneik & $39.82 / 0.9211$ & $40.46 / 0.9376$ & $41.07 / 0.9411$ & $\mathbf{4 5 . 8 3 / 0 . 9 8 1 5}$ & $41.23 / 0.9485$ & $44.74 / 0.9816$ \\
\hline Teapot & $32.57 / 0.7051$ & $33.09 / 0.7218$ & $33.64 / 0.7455$ & $\mathbf{3 5 . 4 3 / 0 . 8 6 0 5}$ & $32.89 / 0.4304$ & $34.24 / 0.8056$ \\
\hline
\end{tabular}

5.10. shows comparisions with the colour (RHF) and scene feature (NFOR) based approaches. We tested across four challenging noisy scene including Dragonfog, Sanmiguel (rendered by $32 \mathrm{spp}$ ) and Sibneik, Teapot (rendered by $16 \mathrm{spp}$ ). Our method (DRMF) shows better result compared with RHF and NFOR in all scenes. In the selected closeups, RHF tends to generate blurry artifices, and NFOR missed few local details although it provides better geometry and texture edge compared with RHF. Our DRMF outperform the previous methods in both preserving the high frequency details and smoothing the noisy artefacts. The error metrics (PSNR and SSIM) of the entire image to compare denoising results of our methods (SRS, DRS, DRM, and DRMF) with RHF and NFOR are in Table 5.3.

\subsubsection{Efficiency comparison}

To denoise an image of $1024^{*} 1024$ pixels, our denoising network (DRMF) requires around 16 seconds to produce the final result. The running time is linearly related with the image resolution. The processing timing for RHF 
is approximately 2 minutes, 20 seconds for LBF, 12 seconds for KPCN, and 5 minutes for NFOR. It is worth noting that the reference rendering of the test scenes requires hundreds of hours rendering time using $5 \mathrm{k}$ to $64 \mathrm{~K}$ samples per pixel. However our denoising filter only needed significantly shorter time (16 second) to denoise images generated by highly limited samples (8 spp to $32 \mathrm{spp}$ ) and achieved almost similar visual quality compared with the reference rendering.

\subsection{Summary and Discussion}

In this chapter, we have presented a pilot study of using deep convolutional networks for denoising path tracing. Specifically, we introduced a multi-scale filter into convolution layer, which can better preserve short and long contextual information within the rendering. By adopting the residual learning strategy, we implemented a separated residual learning manner, and the result shows better denoising quality. To exploit and utilize the auxiliary scene features of the rendering, a virtual flash scene feature is generated for providing detailed information about the scene. Our results demonstrated that a CNN can represent the underlying, fundamental relationship between the noisy and reference rendering. Although it uses a relatively straightforward architecture, our solution is fast and it shows better or similar performance compared with other state-of-the-art denoising techniques for path tracing.

Based on the experiments, the proposed denoising network can generate promissing results for various scene effects, but it is still hard to denoise some challenging scenes like the Villa scene. As discussed in Chapter 3, the light source in this scene is located outside and a few samples per pixel could not successfully compute the connection between the indoor surface point and the outside light source, therefore it fails to provide enough information for the denoising network. In Figure 5.11, under a fixed time budget (1.8 hours), the pixel recovery method in previous 
chapter outperformed the proposed denoising network.

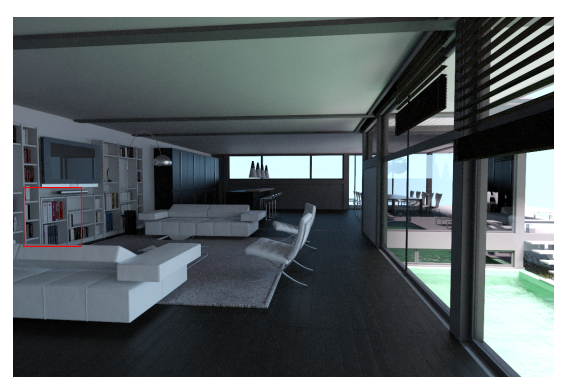

Villa

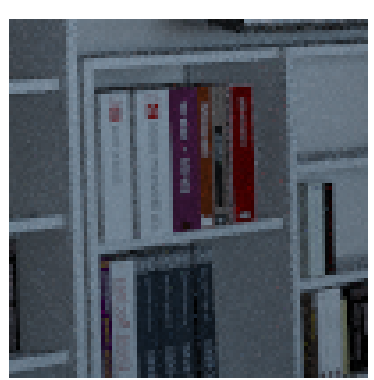

PAWNNM

PSNR: 36.57

SSIM: 0.945

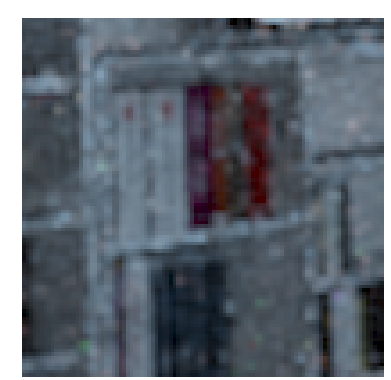

MRDN

PSNR: 29.35 SSIM: 0.871

Figure 5.11: Under a fixed time budget, pixel recovery (in the middle) can generate better visual quality results than the proposed denoising network(on the right).

Looking ahead, one potential task is a complete exploration of the error metrics, which are quite different from each other. Also, combining the perceptual loss into the current loss function in our network, which may reveal better performance during the training. Additionally, different setting of the hyperparameters have been explored for an optimal performance of the network, but a complete investigation for the parameter selection can achieve better solutions. Additional data is always helpful for better network training but our procedure is limited by the huge computation time to render reference images (e.g., days to complete a single image with 64K spp). Recently proposed Noise2Noise learning [115] can relax this limitation by allowing network parameters to be trained using noisy image pairs instead of noise and clean data pairs. Finally, the proposed network only handles the single image denoising, but it would also be helpful for dealt with animation frames, especially in feature film production. 


\section{Chapter 6}

\section{Conclusions and Future Work}

This thesis seeks to address the problem of the increasing time budget in path tracing and tackle this problem using reconstruction and denoising based approaches. We began by laying out the theoretical background of our research in Chapter 2 by first introducing the concept of path tracing. We also pointed out the limitation of path tracing, which is its slow convergence. Given this limitation, we then reviewed related work in reconstruction and denoising based methods.

Given the fact that path tracing usually takes a long time, especially with complex scene settings. Visualizing intermediate results of path tracing is challenging since the synthesized image with limited samples or improper sampling usually suffers from distracting noise. In Chapter 3, we presented a novel reconstruction method that efficiently recovers missing data in incompletely rendered images using matrix completion. Specifically, we present novel methods to construct low-rank matrices for incomplete images including missing pixel, missing sub-pixel, and multi-frame scenarios. A convolutional neural network provides fast pre-completion for initializing missing values, and subsequent weighted nuclear norm minimization with a parameter adjustment strategy efficiently recovers missing values even in high-frequency details. The result shows better visual quality than recent methods including compressed sensing based 
reconstruction. Our method shows promising recovery results even in challenging scenes including high-frequency details.

The second work of this thesis is built upon Chapter 3. In Chapter 4 , we extend the previous method by applying a block Toeplitz structure while forming an enhanced low-rank matrix of pixel recovery, and tensor structure for multi-frame restoration. In this manner, the reconstruction time can be further reduced for both tasks. Besides that, by exploiting temporal coherence of multi-frame with a tensor structure, better recovery quality can be achieved compared to our previous approach.

For the last work of the thesis, we looked into denoising based technique for path tracing. In Chapter 5, we introduce a convolutional neural network based filter for denoising rendered images. The deep neural network has demonstrated success in filtering noise of path tracing rendering under a low sample budget, and the recovery speed is 30 times faster than the proposed reconstruction method in Chapter 4. Unlike a plain CNN that applies fixed kernel size in each layer, we propose a multi-scale residual network with various auxiliary scene features to leverage a new efficient denoising filter for path tracing. Our experimental results show better or similar performance in terms of denoising quality and, speed compare to the state-of-the-art path tracing denoiser.

In the context of efficient solutions for previsualization of path tracing, this thesis covers both reconstruction and denoising based approaches. The methods we presented in this thesis made several novel contributions to intermediate rendering visualization or production-ready application. We believe this research enhanced our understanding of two postprocessing paths for efficient path tracing and will give insights into future rendering research. Contributions of this thesis are provided as follows, along with limitations and the potential research areas that this work enables. 


\subsection{Summary of Contributions}

A number of specific research objectives were stated in the introduction. Here we restate these objectives and relate them to the achievements in the thesis:

\section{Develop Reconstruction based Method for Previsualization}

The first contribution of the thesis is a new efficient and accurate reconstruction based method to recover the missing samples of path tracing. Chapter 3 formalizes the recovery of missing data in incompletely rendered images as a matrix completion problem. Specifically, we adopt the low-rank matrix completion scheme for previsualization of path tracing. Although matrix completion and separation have been applied to many lights rendering [22] and inpainting [4] recently, they have not yet been applied to recover sub-pixel samples of rendering or pixel samples in multiple frames. To form a low-rank matrix, we show how to formulate pixel, sub-pixel, and multi-frame sample problems as matrix completion. Our approach contributes to reducing the spatial and temporal noise in reconstructed renderings. Additionally, we have developed novel nuclear norm minimization methods using a multi-weighted approach with a progressive adjustment strategy. Our method has been evaluated against state-ofthe-art methods and shows better results in terms of visual quality including CS based reconstruction. In particular, our method outperforms with limited samples.

Our second contribution is based on Chapter 3, we present novel methods in Chapter 4 to construct enhanced low-rank matrices and low-rank tensor for dealing with missing pixel and multi-frame scenarios respectively. Specifically, we adopted the latest enhanced matrix completion in pixel recovery and tensor completion for multi-frame case. Then we apply weighted nuclear norm minimization with a parameter adjustment strategy, which can more efficiently recover missing values even in high- 
frequency details.

\section{Develop Denoising based Method for Previsualization}

Another contribution of this thesis is the novel denoising based filter technique in Chapter 5. Motivated by the recent deep learning-based works, we propose to apply a multi-scale residual learning based denoising network. Unlike the previous architectures, which apply a single scale filter in the convolutional layer or perform multi-scale concept through down/up scale the feature maps, we use different filters during the convolution step to preserve both long and short contextual information. Additionally, we propose a separated residual learning strategy to predict the noise and generate a virtual flash image as one of the input scene feature buffers. Our method shows on par or better denoising quality compare to the stateof-the-art methods.

\subsection{Limitations}

Although our proposed works in this thesis show promising results compared with other state-of-the-art techniques, our methods do have some limitations.

\section{Limitations of Our Reconstruction based Methods}

Since the performance of matrix completion and tensor completion relies on proper parameters, such as the size and number of low-rank matrices, the 3-D dimensions of a tensor structure and related hyper-parameters of the algorithm. Over several experiments, we determined appropriate parameters for different applications, but careful analysis of scene information may provide better optimal choices. However, it is challenging to do this because, in general, detailed scene information cannot be known be- 
fore a significant number of samples have been taken. Our reconstruction based method is challenged when attempting to recover values under very low sampling rates $(<10 \%)$. Previous recovery methods, such as the CS based approaches, suffer from similar issues. Our method shows better and more reliable results than CS based approaches at very low sampling rates.

On the other hand, the current implementation of the sub-pixel recovery is not efficient compared with previous filtering methods in terms of the total processing time. The reconstruction of unknown ray samples mainly rely on uniform sampling pattern, which is not the optimal choice to deal with various scenes.

\section{Limitations of Our Denoising based Method}

Our neural network based denoising filter can generate noise-free results efficiently and requires few parameters to manually set, which can be easily integrated into production application. Our learning based approach also have several inherent limitations. Firstly, our denoised result may lack the scene details which are not captured in the input scene features, and it will be missing in our training set. Also, due to these details didn't appear in the training dataset, the network cannot approximate them with mere use of the color information. But the problem can be relieved through adding similar examples to the training set. Similarly, examples with various distributed effects of the test set should be included during training, otherwise the network will fail to remove the corresponding noise.

\subsection{Future Work}

The promising results from this thesis suggest that further development is a worthy cause. These methods are not production ready due to the scenes used in all experiments are not from the visual effects industry, and 
should naturally undergo further research, development, and testing with real production data set. The most interesting avenue for future work in reconstruction based method would be to explore GPU based implementation for better efficiency, since our reconstruction based methods are fully independent across patches or frames, and therefore each recovery step could be computed in parallel on the GPU. Also, integrating neural network techniques in Nvidia's latest ray racing based hardware (e.g., RTX $2080 \mathrm{Ti}$ ) for previsualization of path tracing is worth exploring in future.

Finally, to further make improvement on the performance of our denoising network, several network design choices could be investigated. The first straightforward direction is to explore different network structure and concepts such as autoencoders, generative adversarial networks and U-net, which have demonstrated great success for natural image denoising. All these novel network architectures can potentially improve the performance. Secondly, integrating the perceptual loss into the standard error metrics, and more potential loos functions can be considered. Moreover, it would be helpful in production to extend the proposed denoising network in animation frames noise removal, since the results from Chapter 5 only target for single image denoising. 


\section{Appendix A}

\section{Pre-completion with TV and CNN}

A visual quality comparison of pre-completion in Section 3.2.1 between $\mathrm{TV}$ and our CNN for seven different scenes, all the inputs are only $10 \%$ pixels of the corresponding scene. 
Bamboo Scene

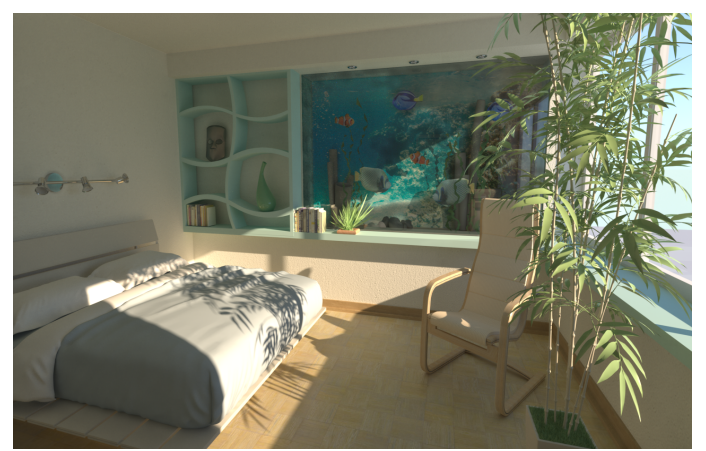

Reference

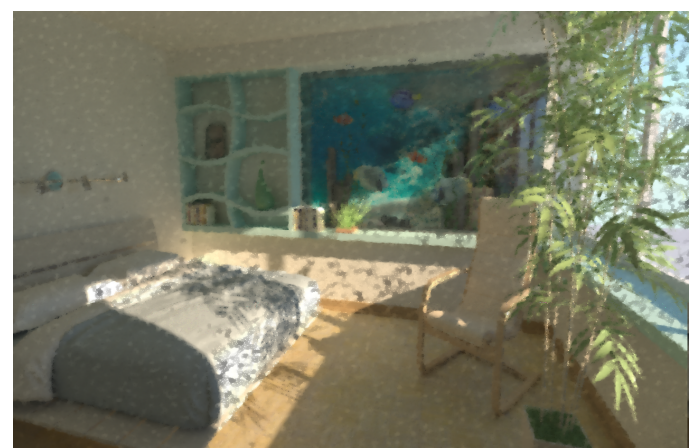

Pre-completion by TV

$$
(\mathrm{psnr}=23.01)
$$

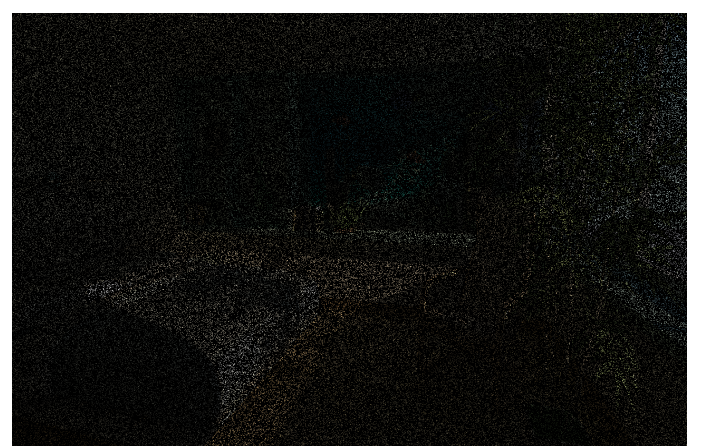

Input $10 \%$ pixels

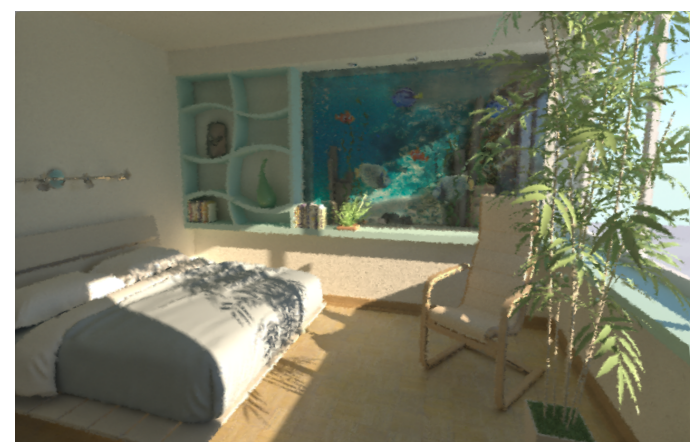

Pre-completion by CNN $(\mathrm{psnr}=26.03)$

Bedroom Scene

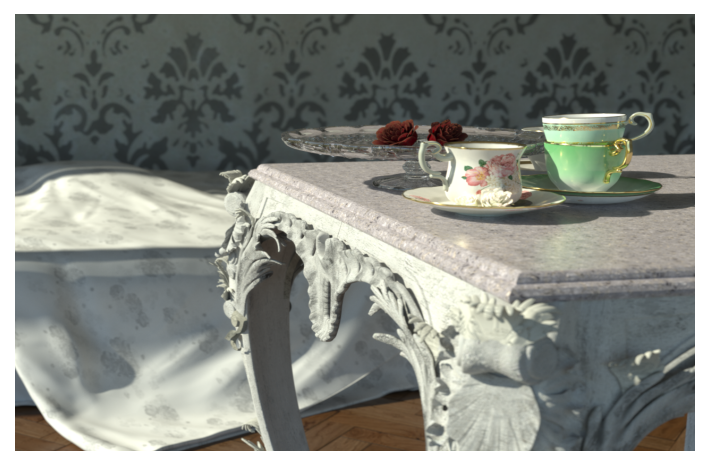

Reference

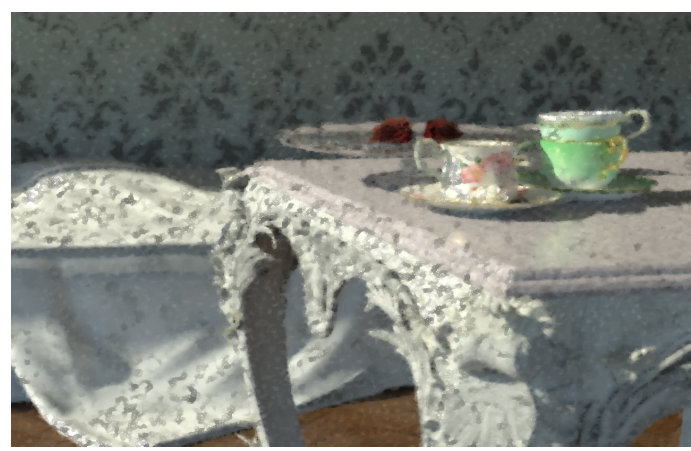

Pre-completion by TV

$(\mathrm{psnr}=23.91)$

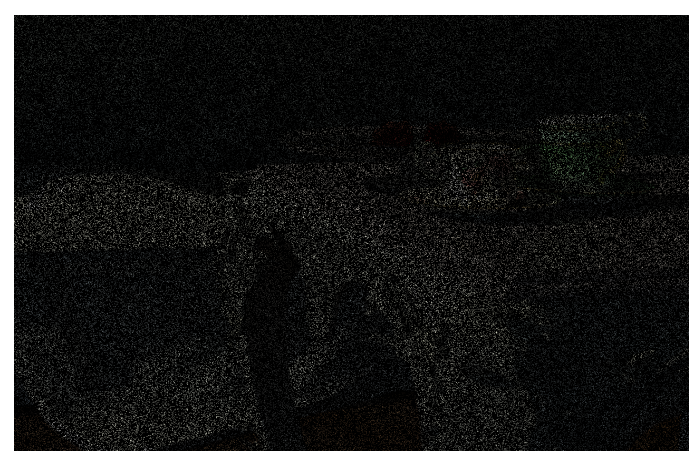

Input $10 \%$ pixels

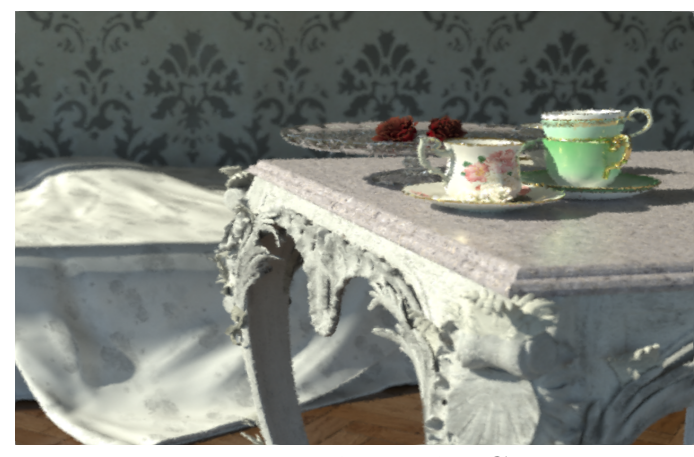

Pre-completion by CNN

$($ psnr $=31.82)$ 
Bottles Scene

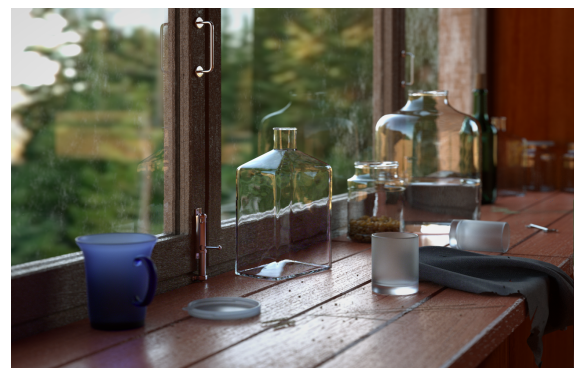

Reference

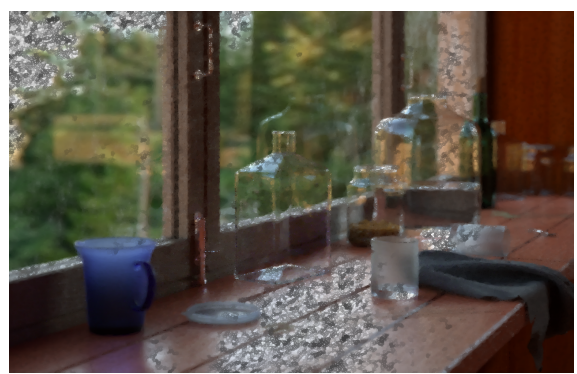

Pre-completion by TV

(psnr=19.73)

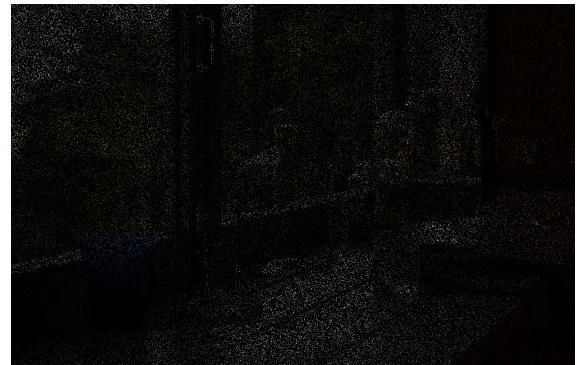

Input $10 \%$ pixels

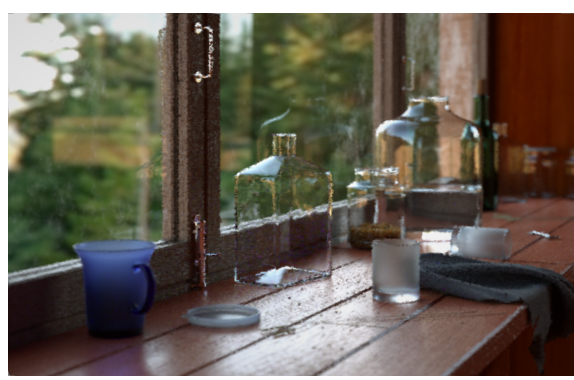

Pre-completion by CNN

(psnr $=30.17$ )

Conference Scene

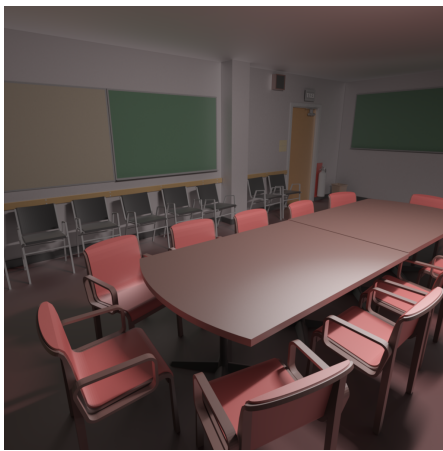

Refernece

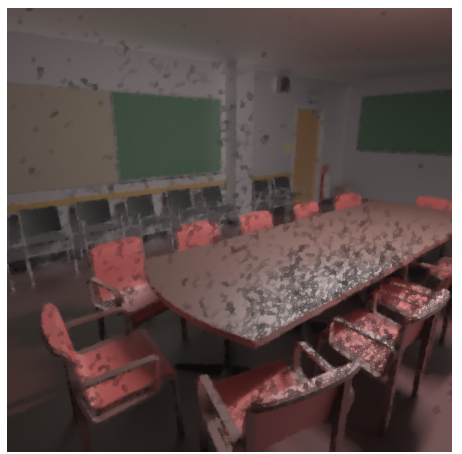

Pre-completion by TV (psnr=25.54)

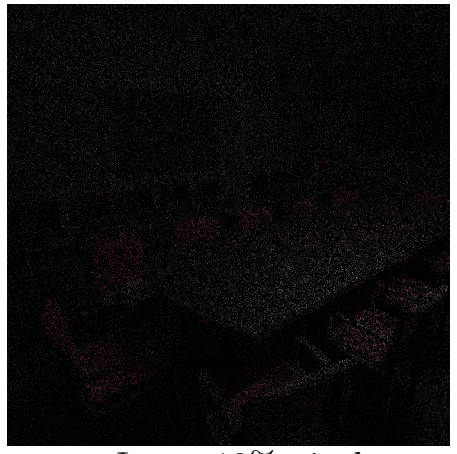

Input $10 \%$ pixels

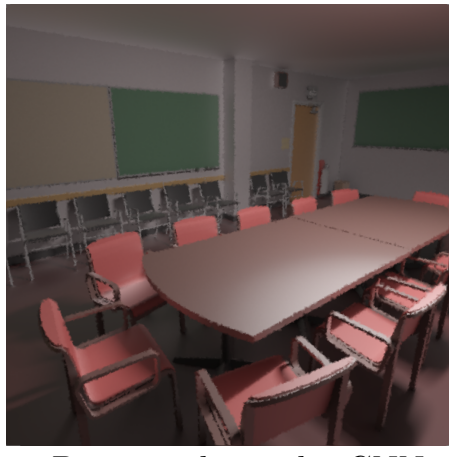

Pre-completion by CNN $($ psnr $=33.67)$ 
Killines Scene

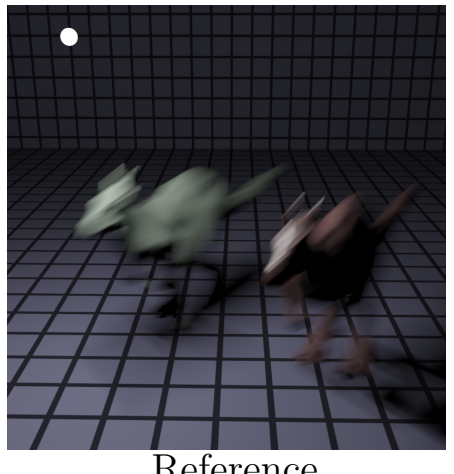

Reference

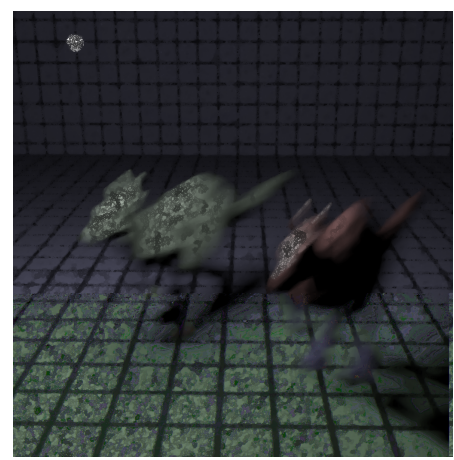

Pre-completion by TV $(\mathrm{psnr}=23.34)$

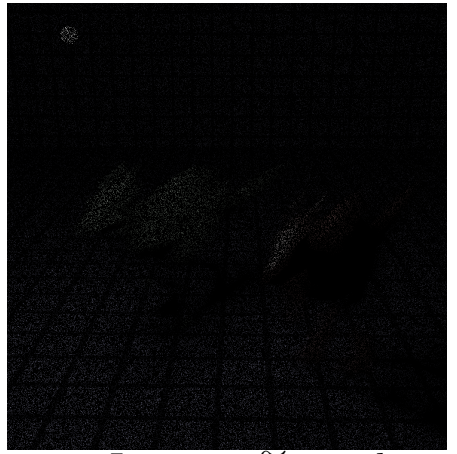

Input $10 \%$ pixels

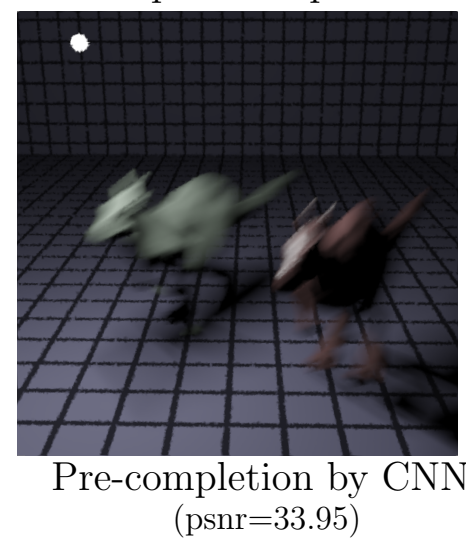

Sanmiguel Scene

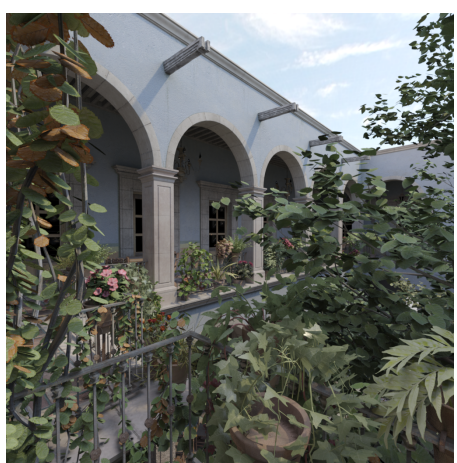

Reference

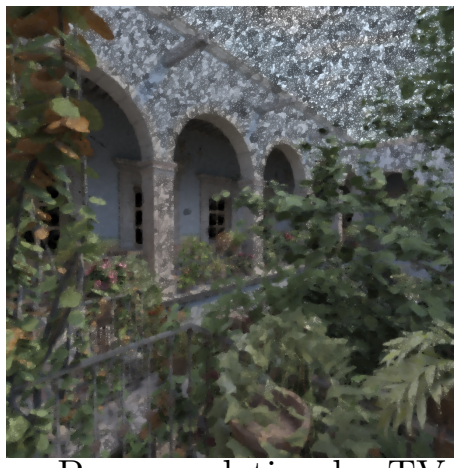

Pre-completion
(psnr $=15.78)$

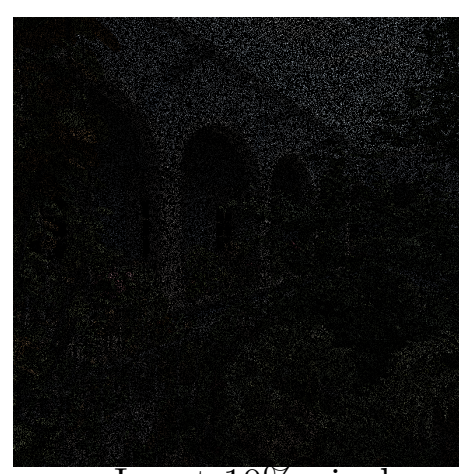

Input $10 \%$ pixels

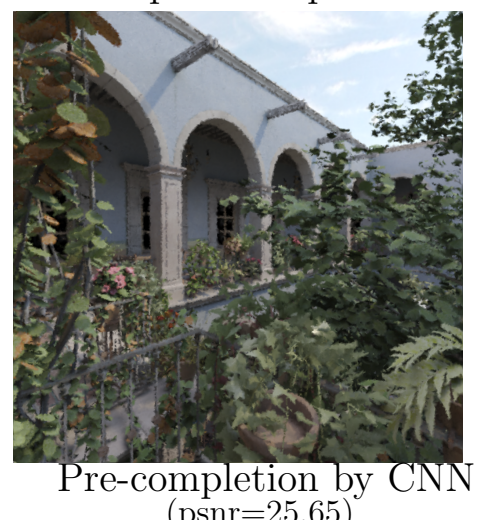

(psnr=25.65) 


\section{Toasters Scene}

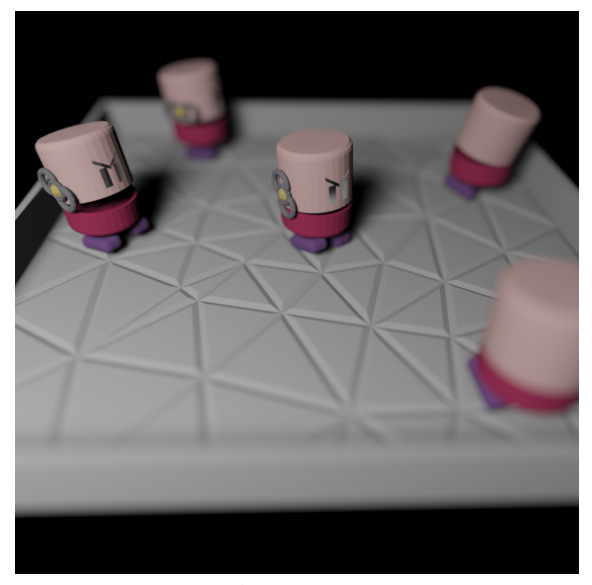

Reference

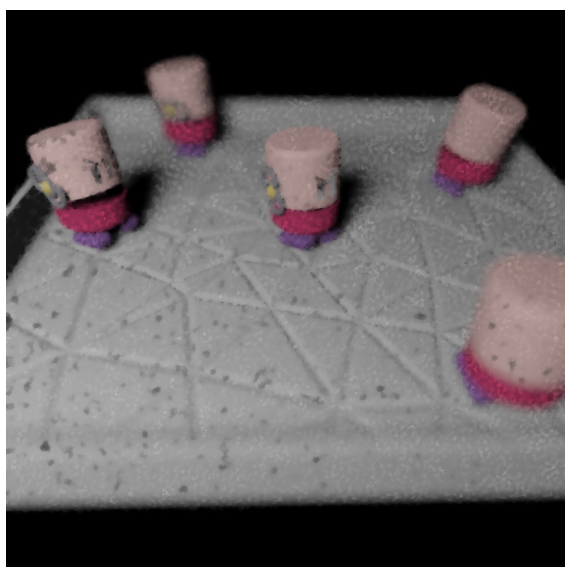

Pre-completion by TV $(\mathrm{psnr}=30.59)$

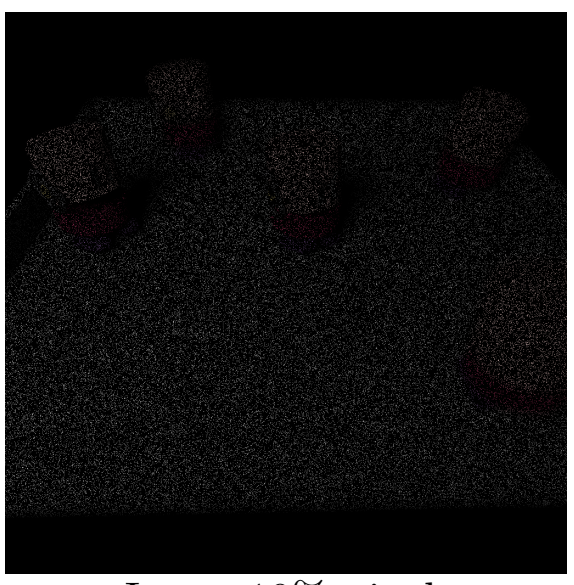

Input $10 \%$ pixels

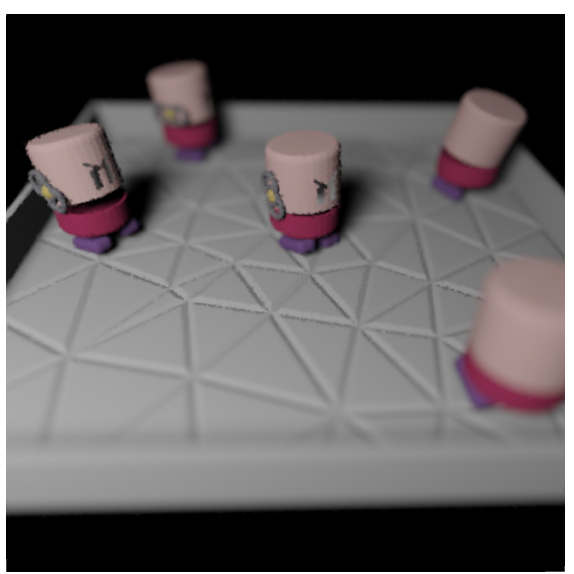

Pre-completion by CNN $($ psnr $=39.74)$ 


\section{Appendix B}

\section{PAWNNM for Inpainting}

A visual quality comparison of NLI, GSR, and the proposed PAWNNM in Chapter 3. Five standard test images were selected and compared the recovery results with $20 \%$ and $30 \%$ sampling. 

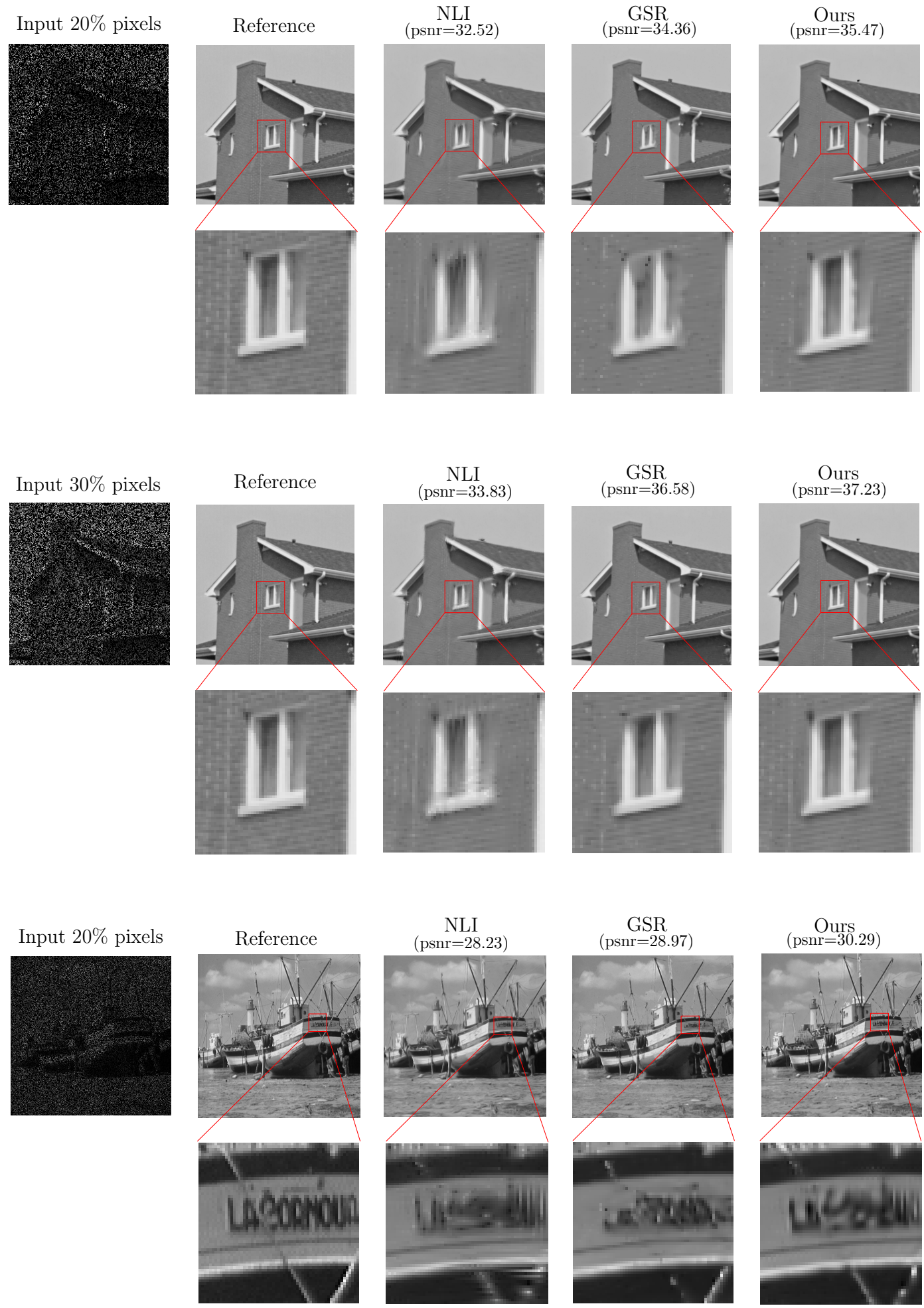

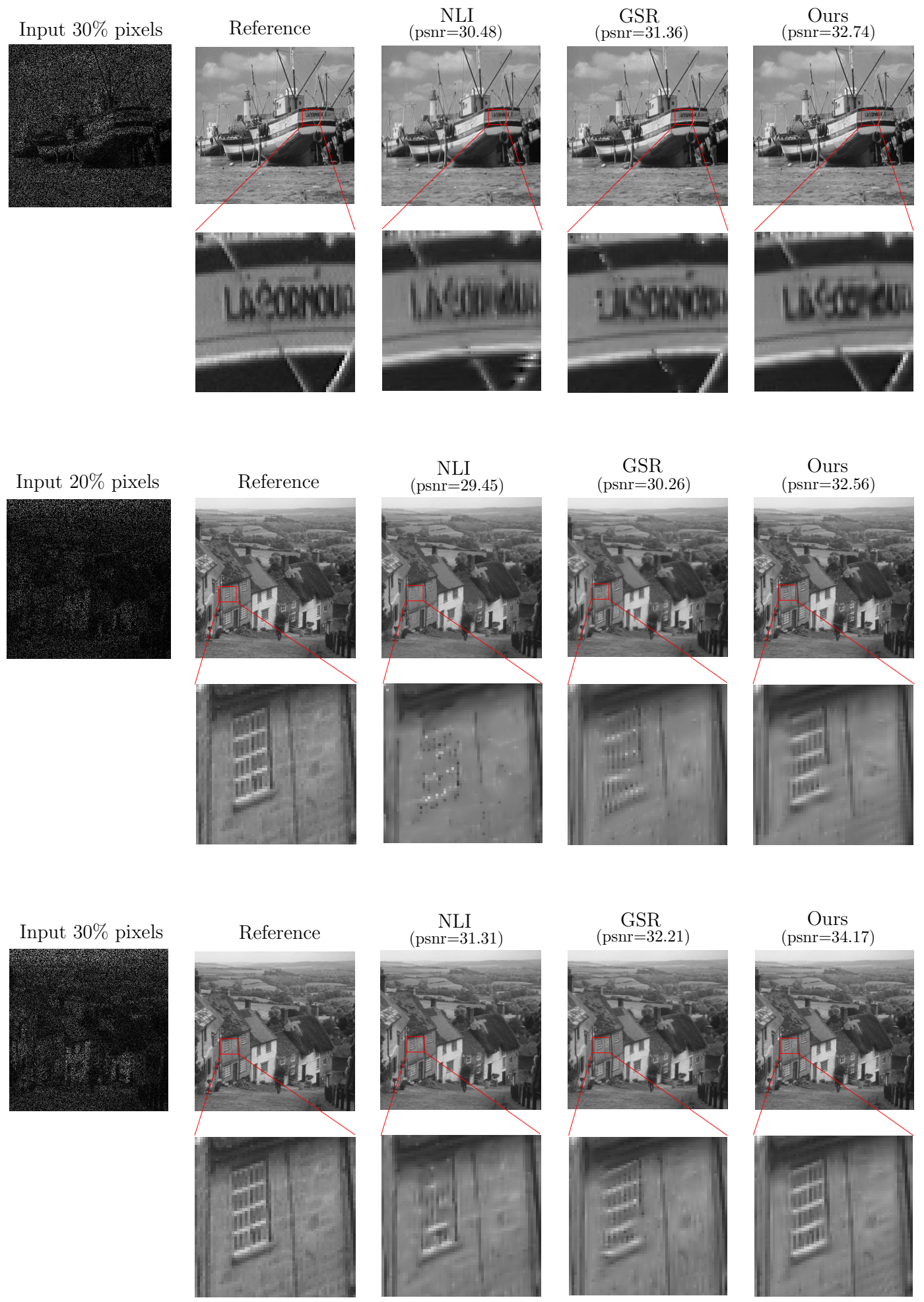

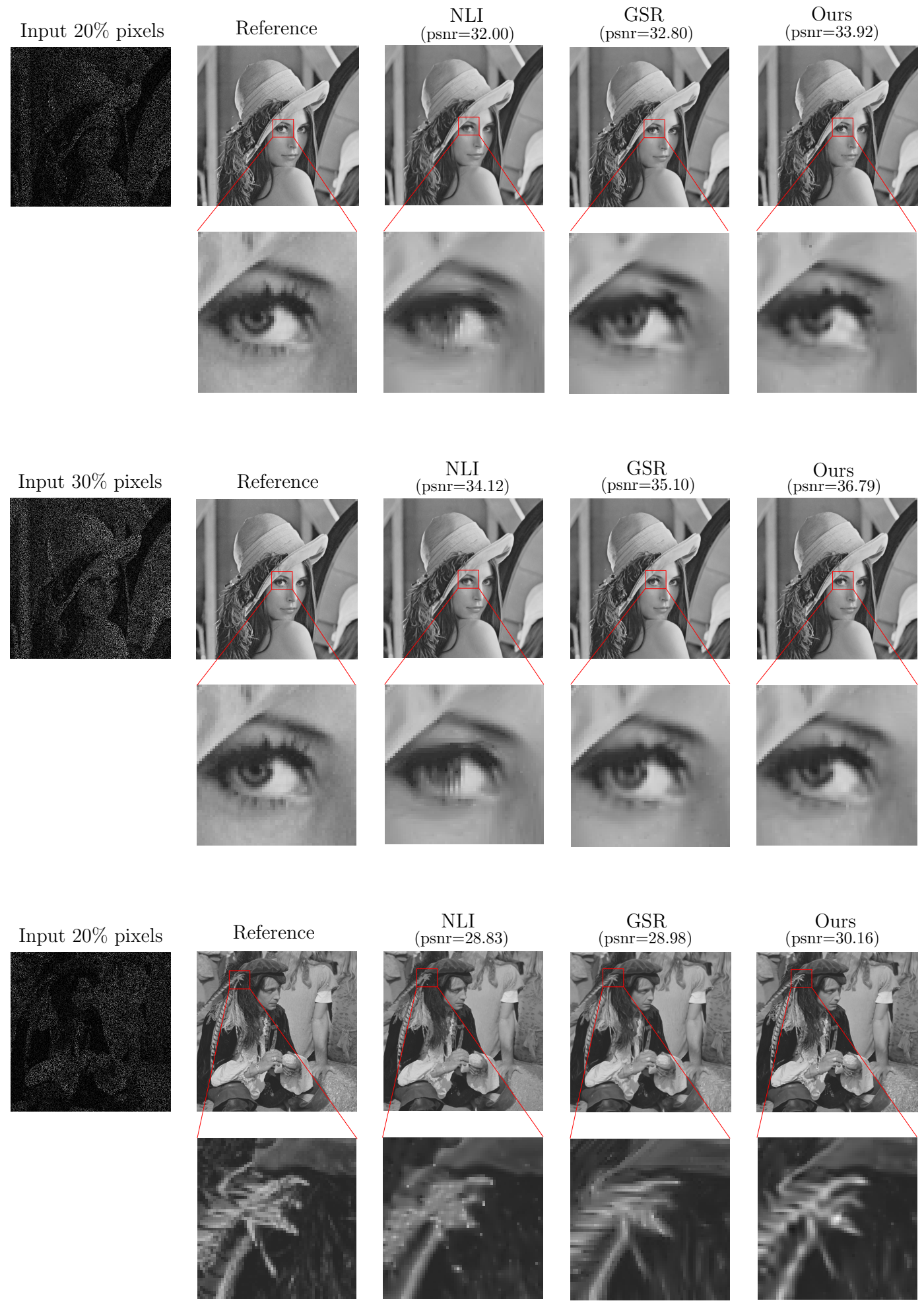

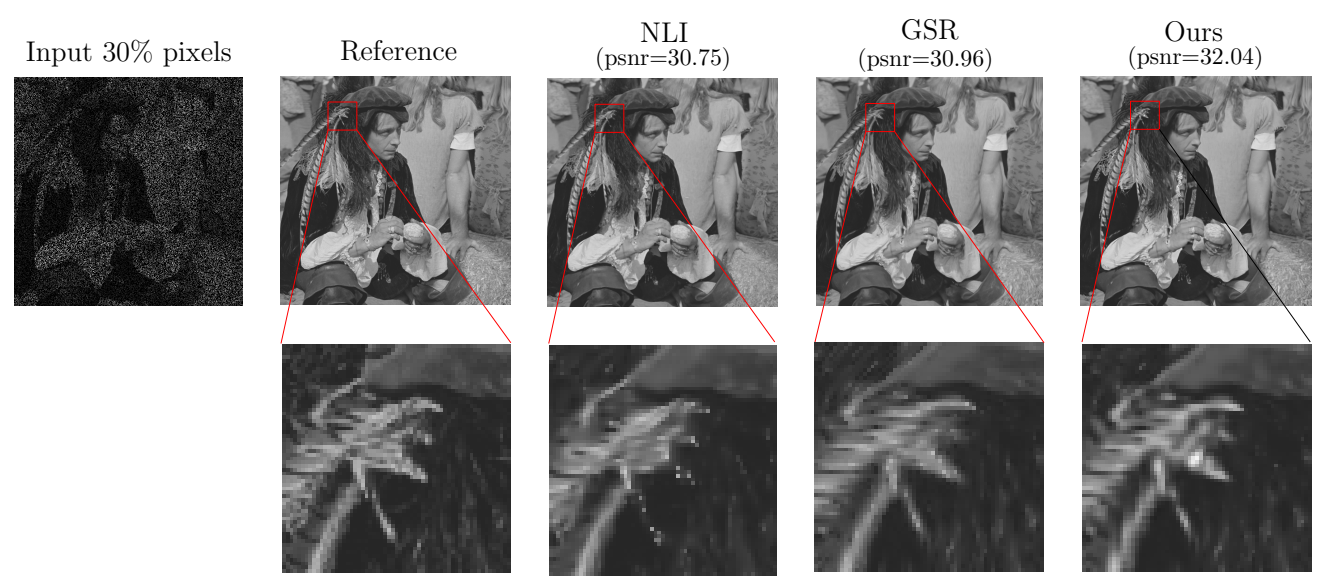


\section{Bibliography}

[1] T.-M. Li, Y.-T. Wu, and Y.-Y. Chuang, "Sure-based optimization for adaptive sampling and reconstruction," ACM Trans. Graph., vol. 31, pp. 194:1-194:9, Nov. 2012.

[2] M. Delbracio, P. Musé, A. Buades, J. Chauvier, N. Phelps, and J.-M. Morel, "Boosting monte carlo rendering by ray histogram fusion," ACM Trans. Graph., vol. 33, pp. 8:1-8:15, Feb. 2014.

[3] N. K. Kalantari, S. Bako, and P. Sen, "A machine learning approach for filtering Monte Carlo noise," ACM Trans. Graph., vol. 34, pp. 122:1-122:12, July 2015.

[4] W. Li, L. Zhao, Z. Lin, D. Xu, and D. Lu, “Non-local image inpainting using low-rank matrix completion," Comput. Graph. Forum, vol. 34, pp. 111-122, Sept. 2015.

[5] J. T. Kajiya, "The rendering equation," in Proceedings of the 13th Annual Conference on Computer Graphics and Interactive Techniques, SIGGRAPH '86, (New York, NY, USA), pp. 143-150, ACM, 1986.

[6] M. Aharon, M. Elad, and A. Bruckstein, "Svdd: An algorithm for designing overcomplete dictionaries for sparse representation," Trans. Sig. Proc., vol. 54, pp. 4311-4322, Nov. 2006. 
[7] H. Takeda, S. Farsiu, and P. Milanfar, "Kernel regression for image processing and reconstruction," IEEE Transactions on Image Processing, vol. 16, pp. 349-366, Feb 2007.

[8] J. Mairal, M. Elad, and G. Sapiro, "Sparse representation for color image restoration," IEEE Transactions on Image Processing, vol. 17, pp. 53-69, Jan 2008.

[9] P. Sen and S. Darabi, "Compressive rendering: A rendering application of compressed sensing," IEEE Transactions on Visualization and Computer Graphics, vol. 17, pp. 487 - 499, April 2011.

[10] H. Dammertz, D. Sewtz, J. Hanika, and H. Lensch, "Edge-avoiding à-trous wavelet transform for fast global illumination filtering," in Proceedings of the Conference on High Performance Graphics, pp. 67-75, Eurographics Association, 2010.

[11] B. Moon, N. Carr, and S.-E. Yoon, "Adaptive rendering based on weighted local regression," ACM Transactions on Graphics (TOG), vol. 33, no. 5, pp. 170:1-170:14, 2014.

[12] F. Rousselle, C. Knaus, and M. Zwicker, "Adaptive sampling and reconstruction using greedy error minimization," in ACM Transactions on Graphics (TOG), vol. 30, pp. 159:1-159:12, ACM, 2011.

[13] F. Rousselle, C. Knaus, and M. Zwicker, "Adaptive rendering with non-local means filtering," ACM Transactions on Graphics (TOG), vol. 31, no. 6, pp. 195:1-195:11, 2012.

[14] F. Rousselle, M. Manzi, and M. Zwicker, "Robust denoising using feature and color information," in Computer Graphics Forum, vol. 32, pp. 121-130, Wiley Online Library, 2013.

[15] P. Sen and S. Darabi, "On filtering the noise from the random parameters in monte carlo rendering.," ACM Trans. Graph., vol. 31, no. 3, pp. 18:1-18:15, 2012. 
[16] E. J. Candès, X. Li, Y. Ma, and J. Wright, "Robust principal component analysis?," Journal of the ACM (JACM), vol. 58, no. 3, pp. 11:111:37, 2011.

[17] E. J. Candès and B. Recht, "Exact matrix completion via convex optimization," Foundations of Computational mathematics, vol. 9, no. 6, pp. 717-772, 2009.

[18] M. E. Kilmer, K. Braman, N. Hao, and R. C. Hoover, "Third-order tensors as operators on matrices: A theoretical and computational framework with applications in imaging," SIAM Journal on Matrix Analysis and Applications, vol. 34, no. 1, pp. 148-172, 2013.

[19] J. Liu, P. Musialski, P. Wonka, and J. Ye, "Tensor completion for estimating missing values in visual data," IEEE Transactions on Pattern Analysis and Machine Intelligence, vol. 35, no. 1, pp. 208-220, 2013.

[20] M. Lustig, D. Donoho, and J. M. Pauly, "Sparse MRI: The application of compressed sensing for rapid MR imaging," Magnetic resonance in medicine, vol. 58, no. 6, pp. 1182-1195, 2007.

[21] M. Wakin, J. N. Laska, M. F. Duarte, D. Baron, S. Sarvotham, D. Takhar, K. F. Kelly, and R. G. Baraniuk, "Compressive imaging for video representation and coding," in Picture Coding Symposium, vol. 1, p. 13, 2006.

[22] Y. Huo, R. Wang, S. Jin, X. Liu, and H. Bao, "A matrix sampling-andrecovery approach for many-lights rendering," ACM Trans. Graph., vol. 34, pp. 210:1-210:12, Oct. 2015.

[23] E. Miandji, J. Kronander, and J. Unger, "Compressive image reconstruction in reduced union of subspaces," vol. 34, (Chichester, UK), pp. 33-44, The Eurographs Association \&\#38; John Wiley \&\#38; Sons, Ltd., May 2015. 
[24] M. Bertalmio, G. Sapiro, V. Caselles, and C. Ballester, "Image inpainting," in Proceedings of the 27th Annual Conference on Computer Graphics and Interactive Techniques, SIGGRAPH '00, pp. 417-424, 2000.

[25] A. Bugeau, M. Bertalmío, V. Caselles, and G. Sapiro, "A comprehensive framework for image inpainting," IEEE Transactions on Image Processing, vol. 19, no. 10, pp. 2634-2645, 2010.

[26] T. F. Chan and J. Shen, "Non-texture inpainting by curvature-driven diffusions (cdd)," J. Vis. Comun. Image Represent., vol. 12, pp. 436449, Dec. 2001.

[27] D. Tschumperle and R. Deriche, "Vector-valued image regularization with pdes: A common framework for different applications," IEEE transactions on pattern analysis and machine intelligence, vol. 27, no. 4, pp. 506-517, 2005.

[28] J. Sun, L. Yuan, J. Jia, and H.-Y. Shum, "Image completion with structure propagation," in ACM Transactions on Graphics (ToG), vol. 24, pp. 861-868, ACM, 2005.

[29] M. M. O. B. B. Richard and M. Y.-S. Chang, "Fast digital image inpainting," in Appeared in the Proceedings of the International Conference on Visualization, Imaging and Image Processing (VIIP 2001), Marbella, Spain, pp. 106-107, 2001.

[30] A. Telea, "An image inpainting technique based on the fast marching method," Journal of graphics tools, vol. 9, no. 1, pp. 23-34, 2004.

[31] B. Yan, Y. Gao, K. Sun, and B. Yang, "Efficient seam carving for object removal," in Image Processing (ICIP), 2013 20th IEEE International Conference on, pp. 1331-1335, IEEE, 2013. 
[32] A. A. Efros and T. K. Leung, "Texture synthesis by non-parametric sampling," in Proceedings of the International Conference on Computer Vision-Volume 2 - Volume 2, ICCV '99, (Washington, DC, USA), pp. 1033-1038, IEEE Computer Society, 1999.

[33] D. J. Heeger and J. R. Bergen, "Pyramid-based texture analysis/synthesis," in Proceedings of the 22nd annual conference on Computer graphics and interactive techniques, pp. 229-238, ACM, 1995.

[34] J. S. De Bonet, "Multiresolution sampling procedure for analysis and synthesis of texture images," in Proceedings of the 24th annual conference on Computer graphics and interactive techniques, pp. 361-368, ACM Press/Addison-Wesley Publishing Co., 1997.

[35] H. Igehy and L. Pereira, "Image replacement through texture synthesis," in Image Processing, 1997. Proceedings., International Conference on, vol. 3, pp. 186-189, IEEE, 1997.

[36] A. Criminisi, P. Pérez, and K. Toyama, "Region filling and object removal by exemplar-based image inpainting," IEEE Transactions on image processing, vol. 13, no. 9, pp. 1200-1212, 2004.

[37] I. Drori, D. Cohen-Or, and H. Yeshurun, "Fragment-based image completion," in ACM Transactions on graphics (TOG), vol. 22, pp. 303312, ACM, 2003.

[38] C. Guillemot, M. Turkan, O. Le Meur, and M. Ebdelli, "Image inpainting using lle-ldnr and linear subspace mappings," in 2013 IEEE International Conference on Acoustics, Speech and Signal Processing, pp. 1558-1562, IEEE, 2013.

[39] J. Hays and A. A. Efros, "Scene completion using millions of photographs," in ACM Transactions on Graphics (TOG), vol. 26, pp. 87-94, ACM, 2007. 
[40] O. Le Meur and C. Guillemot, "Super-resolution-based inpainting," in European Conference on Computer Vision, pp. 554-567, Springer, 2012.

[41] Z. Xu and J. Sun, "Image inpainting by patch propagation using patch sparsity," IEEE transactions on image processing, vol. 19, no. 5, pp. 1153-1165, 2010.

[42] J.-F. Aujol, S. Ladjal, and S. Masnou, "Exemplar-based inpainting from a variational point of view," SIAM Journal on Mathematical Analysis, vol. 42, no. 3, pp. 1246-1285, 2010.

[43] M. Bertalmio, L. Vese, G. Sapiro, and S. Osher, "Simultaneous structure and texture image inpainting," IEEE transactions on image processing, vol. 12, no. 8, pp. 882-889, 2003.

[44] A. A. Efros and W. T. Freeman, "Image quilting for texture synthesis and transfer," in Proceedings of the 28th annual conference on Computer graphics and interactive techniques, pp. 341-346, ACM, 2001.

[45] L. Atzori and F. G. De Natale, "Error concealment in video transmission over packet networks by a sketch-based approach," Signal processing: image communication, vol. 15, no. 1-2, pp. 57-76, 1999.

[46] A. Rares, M. J. Reinders, and J. Biemond, "Edge-based image restoration," IEEE Transactions on Image Processing, vol. 14, no. 10, pp. 1454-1468, 2005.

[47] E. J. Candès, "The restricted isometry property and its implications for compressed sensing," Comptes Rendus Mathematique, vol. 346, no. 9, pp. 589-592, 2008.

[48] K. Marwah, G. Wetzstein, Y. Bando, and R. Raskar, “Compressive light field photography using overcomplete dictionaries and optimized projections," ACM Transactions on Graphics (TOG), vol. 32, no. 4, pp. 46:1-46:12, 2013. 
[49] L. Zelnik-Manor, K. Rosenblum, and Y. C. Eldar, "Dictionary optimization for block-sparse representations," IEEE Transactions on Signal Processing, vol. 60, no. 5, pp. 2386-2395, 2012.

[50] J.-F. Cai, E. J. Candès, and Z. Shen, "A singular value thresholding algorithm for matrix completion," SIAM Journal on Optimization, vol. 20, no. 4, pp. 1956-1982, 2010.

[51] M. E. Lee and R. A. Redner, "A note on the use of nonlinear filtering in computer graphics," IEEE Computer Graphics and Applications, vol. 10, no. 3, pp. 23-29, 1990.

[52] H. W. Jensen and N. J. Christensen, "Optimizing path tracing using noise reduction filters," 1995.

[53] P. Choudhury and J. Tumblin, "The trilateral filter for high contrast images and meshes," in ACM SIGGRAPH 2005 Courses, pp. 186-196, ACM, 2005.

[54] R. Xu and S. N. Pattanaik, "A novel monte carlo noise reduction operator," IEEE Computer Graphics and Applications, vol. 25, no. 2, pp. 31-35, 2005.

[55] K. Bala, B. Walter, and D. P. Greenberg, "Combining edges and points for interactive high-quality rendering," in ACM Transactions on Graphics (TOG), vol. 22, pp. 631-640, ACM, 2003.

[56] K. Egan, Y.-T. Tseng, N. Holzschuch, F. Durand, and R. Ramamoorthi, "Frequency analysis and sheared reconstruction for rendering motion blur," in ACM Transactions on Graphics (TOG), vol. 28, pp. 93:1-93:13, ACM, 2009.

[57] S. U. Mehta, B. Wang, and R. Ramamoorthi, "Axis-aligned filtering for interactive sampled soft shadows," ACM Transactions on Graphics (TOG), vol. 31, no. 6, pp. 163:1-163:10, 2012. 
[58] H. E. Rushmeier and G. J. Ward, "Energy preserving non-linear filters," in Proceedings of the 21st Annual Conference on Computer Graphics and Interactive Techniques, SIGGRAPH '94, (New York, NY, USA), pp. 131-138, ACM, 1994.

[59] M. D. McCool, "Anisotropic diffusion for monte carlo noise reduction," ACM Transactions on Graphics (TOG), vol. 18, no. 2, pp. 171194, 1999.

[60] Q. Xu, Y. Liu, R. Zhang, S. Bao, R. Scopigno, and M. Sbert, "Noise reduction for path traced imaging of participating media," in Signal Processing Conference, 2011 19th European, pp. 1683-1687, IEEE, 2011.

[61] R. S. Overbeck, C. Donner, and R. Ramamoorthi, "Adaptive wavelet rendering.," ACM Trans. Graph., vol. 28, no. 5, pp. 140:1-140:12, 2009.

[62] F. Rousselle, C. Knaus, and M. Zwicker, "Adaptive sampling and reconstruction using greedy error minimization," in Proceedings of the 2011 SIGGRAPH Asia Conference, SA '11, (New York, NY, USA), pp. 159:1-159:12, ACM, 2011.

[63] A. Buades, B. Coll, and J.-M. Morel, "A review of image denoising algorithms, with a new one," Multiscale Modeling $\mathcal{E}$ Simulation, vol. 4, no. 2, pp. 490-530, 2005.

[64] N. K. Kalantari and P. Sen, "Removing the noise in monte carlo rendering with general image denoising algorithms," in Computer Graphics Forum, vol. 32, pp. 93-102, Wiley Online Library, 2013.

[65] S. Laine, H. Saransaari, J. Kontkanen, J. Lehtinen, and T. Aila, "Incremental instant radiosity for real-time indirect illumination," in Proceedings of the 18th Eurographics conference on Rendering Techniques, pp. 277-286, Eurographics Association, 2007. 
[66] B. Segovia, J. C. Iehl, R. Mitanchey, and B. Péroche, "Noninterleaved deferred shading of interleaved sample patterns," in Graphics Hardware, pp. 53-60, 2006.

[67] P. Bauszat, M. Eisemann, and M. Magnor, "Guided image filtering for interactive high-quality global illumination," in Computer Graphics Forum, vol. 30, pp. 1361-1368, Wiley Online Library, 2011.

[68] P. Shirley, T. Aila, J. Cohen, E. Enderton, S. Laine, D. Luebke, and M. McGuire, "A local image reconstruction algorithm for stochastic rendering," in Symposium on Interactive 3D Graphics and Games, pp. 9-14, ACM, 2011.

[69] Y. Chen and Y. Chi, "Spectral compressed sensing via structured matrix completion," in International Conference on Machine Learning, pp. 414-422, 2013.

[70] C. M. Stein, "Estimation of the mean of a multivariate normal distribution," The annals of Statistics, pp. 1135-1151, 1981.

[71] F. Rousselle, M. Manzi, and M. Zwicker, "Robust denoising using feature and color information," Computer Graphics Forum, vol. 32, no. 7, pp. 121-130, 2013.

[72] P. Sen and S. Darabi, "On filtering the noise from the random parameters in Monte Carlo rendering," ACM Trans. Graph., vol. 31, pp. 18:1-18:15, May 2012.

[73] J. Lehtinen, T. Aila, J. Chen, S. Laine, and F. Durand, "Temporal light field reconstruction for rendering distribution effects," in $A C M$ Transactions on Graphics (TOG), vol. 30, pp. 55:1-55:12, ACM, 2011.

[74] J. Lehtinen, T. Aila, S. Laine, and F. Durand, "Reconstructing the indirect light field for global illumination," $A C M$ Transactions on Graphics (TOG), vol. 31, no. 4, pp. 51:1-51:10, 2012. 
[75] A. v. d. Oord, S. Dieleman, H. Zen, K. Simonyan, O. Vinyals, A. Graves, N. Kalchbrenner, A. Senior, and K. Kavukcuoglu, "Wavenet: A generative model for raw audio," arXiv preprint arXiv:1609.03499, 2016.

[76] K. He, X. Zhang, S. Ren, and J. Sun, "Deep residual learning for image recognition," in Proceedings of the IEEE conference on computer vision and pattern recognition, pp. 770-778, 2016.

[77] J. Xie, L. Xu, and E. Chen, "Image denoising and inpainting with deep neural networks," in Advances in neural information processing systems, pp. 341-349, 2012.

[78] K. Zhang, W. Zuo, Y. Chen, D. Meng, and L. Zhang, "Beyond a gaussian denoiser: Residual learning of deep cnn for image denoising," IEEE Transactions on Image Processing, vol. 26, no. 7, pp. 3142-3155, 2017.

[79] W. Yang, J. Feng, J. Yang, F. Zhao, J. Liu, Z. Guo, and S. Yan, "Deep edge guided recurrent residual learning for image superresolution," IEEE Transactions on Image Processing, vol. 26, no. 12, pp. 5895-5907, 2017.

[80] N. K. Kalantari, S. Bako, and P. Sen, "A machine learning approach for filtering monte carlo noise.," ACM Trans. Graph., vol. 34, no. 4, pp. 122:1-122:12, 2015.

[81] S. Bako, T. Vogels, B. McWilliams, M. Meyer, J. Novák, A. Harvill, P. Sen, T. Derose, and F. Rousselle, "Kernel-predicting convolutional networks for denoising monte carlo renderings," ACM Trans. Graph, vol. 36, no. 4, pp. 97:1-97:14, 2017.

[82] T. Vogels, F. Rousselle, B. Mcwilliams, G. Röthlin, A. Harvill, D. Adler, M. Meyer, and J. Novák, “Denoising with kernel predic- 
tion and asymmetric loss functions," ACM Transactions on Graphics (TOG), vol. 37, no. 4, pp. 124:1-124:15, 2018.

[83] C. R. A. Chaitanya, A. S. Kaplanyan, C. Schied, M. Salvi, A. Lefohn, D. Nowrouzezahrai, and T. Aila, "Interactive reconstruction of monte carlo image sequences using a recurrent denoising autoencoder," ACM Transactions on Graphics (TOG), vol. 36, no. 4, pp. 98:198:12, 2017.

[84] J. Wright, A. Ganesh, S. Rao, Y. Peng, and Y. Ma, "Robust principal component analysis: Exact recovery of corrupted low-rank matrices via convex optimization," in Advances in neural information processing systems, pp. 2080-2088, 2009.

[85] Z. Zhang, X. Liang, A. Ganesh, and Y. Ma, "Tilt: transform invariant low-rank textures," in Computer Vision-ACCV 2010, pp. 314-328, Springer, 2011.

[86] G. Liu, Z. Lin, and Y. Yu, "Robust subspace segmentation by lowrank representation," in Proceedings of the 27th international conference on machine learning (ICML-10), pp. 663-670, 2010.

[87] K. Dabov, A. Foi, V. Katkovnik, and K. Egiazarian, “Image denoising by sparse 3-d transform-domain collaborative filtering," Image Processing, IEEE Transactions on, vol. 16, no. 8, pp. 2080-2095, 2007.

[88] Y. Wang, J. Yang, W. Yin, and Y. Zhang, "A new alternating minimization algorithm for total variation image reconstruction," SIAM Journal on Imaging Sciences, vol. 1, no. 3, pp. 248-272, 2008.

[89] A. Krizhevsky, I. Sutskever, and G. E. Hinton, "Imagenet classification with deep convolutional neural networks," in Advances in neural information processing systems, pp. 1097-1105, 2012. 
[90] S. Gu, L. Zhang, W. Zuo, and X. Feng, “Weighted nuclear norm minimization with application to image denoising," in 2014 IEEE Conference on Computer Vision and Pattern Recognition, pp. 2862-2869, June 2014.

[91] L. Mancera and J. Portilla, "Non-convex sparse optimization through deterministic annealing and applications," in 2008 15th IEEE International Conference on Image Processing, pp. 917-920, Oct 2008 .

[92] J. Zhang, D. Zhao, and W. Gao, “Group-based sparse representation for image restoration," IEEE Transactions on Image Processing, vol. 23, no. 8, pp. 3336-3351, 2014.

[93] N. Li and B. Li, "Tensor completion for on-board compression of hyperspectral images," in Image Processing (ICIP), 2010 17th IEEE International Conference on, pp. 517-520, IEEE, 2010.

[94] L. Serafini and A. d. Garcez, "Logic tensor networks: Deep learning and logical reasoning from data and knowledge," arXiv preprint arXiv:1606.04422, 2016.

[95] R. M. Gray et al., "Toeplitz and circulant matrices: A review," Foundations and Trends $(\mathbb{B}$ in Communications and Information Theory, vol. 2, no. 3, pp. 155-239, 2006.

[96] H. Akaike, "Block toeplitz matrix inversion," SIAM Journal on Applied Mathematics, vol. 24, no. 2, pp. 234-241, 1973.

[97] T. G. Kolda and B. W. Bader, "Tensor decompositions and applications," SIAM review, vol. 51, no. 3, pp. 455-500, 2009.

[98] L. R. Tucker, "Some mathematical notes on three-mode factor analysis," Psychometrika, vol. 31, pp. 279-311, Sep 1966. 
[99] S. Boyd, N. Parikh, E. Chu, B. Peleato, and J. Eckstein, “Distributed optimization and statistical learning via the alternating direction method of multipliers," Found. Trends Mach. Learn., vol. 3, pp. 1-122, Jan. 2011.

[100] M. Pharr and G. Humphreys, Physically based rendering: From theory to implementation. Morgan Kaufmann, 2004.

[101] P. Liu, J. Lewis, and T. Rhee, "Low-rank matrix completion to reconstruct incomplete rendering images," IEEE Transactions on Visualization and Computer Graphics, vol. 24, pp. 2353-2365, Aug 2018.

[102] P. Buyssens, A. Elmoataz, and O. Lézoray, "Multiscale convolutional neural networks for vision-based classification of cells," in Asian Conference on Computer Vision, pp. 342-352, Springer, 2012.

[103] Z. Cai, Q. Fan, R. S. Feris, and N. Vasconcelos, "A unified multi-scale deep convolutional neural network for fast object detection," in European Conference on Computer Vision, pp. 354-370, Springer, 2016.

[104] I. J. Goodfellow, D. Warde-Farley, M. Mirza, A. Courville, and Y. Bengio, "Maxout networks," in Proceedings of the 30th International Conference on International Conference on Machine Learning - Volume 28, ICML'13, pp. III-1319-III-1327, JMLR.org, 2013.

[105] A. Krizhevsky, I. Sutskever, and G. E. Hinton, "Imagenet classification with deep convolutional neural networks," Commun. ACM, vol. 60, pp. 84-90, May 2017.

[106] H. Zhao, O. Gallo, I. Frosio, and J. Kautz, "Loss functions for image restoration with neural networks," IEEE Transactions on Computational Imaging, vol. 3, no. 1, pp. 47-57, 2017.

[107] M. Pharr, W. Jakob, and G. Humphreys, Physically based rendering: From theory to implementation. Morgan Kaufmann, 2016. 
[108] G. Petschnigg, R. Szeliski, M. Agrawala, M. Cohen, H. Hoppe, and K. Toyama, "Digital photography with flash and no-flash image pairs," ACM Trans. Graph., vol. 23, pp. 664-672, Aug. 2004.

[109] E. Eisemann and F. Durand, "Flash photography enhancement via intrinsic relighting," in ACM transactions on graphics (TOG), vol. 23, pp. 673-678, ACM, 2004.

[110] B. Moon, J. Y. Jun, J. Lee, K. Kim, T. Hachisuka, and S.-E. Yoon, “Robust image denoising using a virtual flash image for monte carlo ray tracing," Computer Graphics Forum, vol. 32, no. 1, pp. 139-151.

[111] D. P. Kingma and J. Ba, "Adam: A method for stochastic optimization," arXiv preprint arXiv:1412.6980, 2014.

[112] S. S. Girija, "Tensorflow: Large-scale machine learning on heterogeneous distributed systems," Software available from tensorflow. org, 2016.

[113] M. Delbracio, P. Musé, A. Buades, J. Chauvier, N. Phelps, and J.-M. Morel, "Boosting monte carlo rendering by ray histogram fusion," ACM Transactions on Graphics (TOG), vol. 33, no. 1, p. 8, 2014.

[114] B. Bitterli, F. Rousselle, B. Moon, J. A. Iglesias-Guitián, D. Adler, K. Mitchell, W. Jarosz, and J. Novák, "Nonlinearly weighted firstorder regression for denoising monte carlo renderings," in Computer Graphics Forum, vol. 35, pp. 107-117, Wiley Online Library, 2016.

[115] J. Lehtinen, J. Munkberg, J. Hasselgren, S. Laine, T. Karras, M. Aittala, and T. Aila, "Noise2noise: Learning image restoration without clean data," arXiv preprint arXiv:1803.04189, 2018. 